\title{
COMPACTIFICATIONS OF THE GENERALIZED JACOBIAN VARIETY ${ }^{1}$ \\ BY
}

\author{
TADAO ODA AND C. S. SESHADRI
}

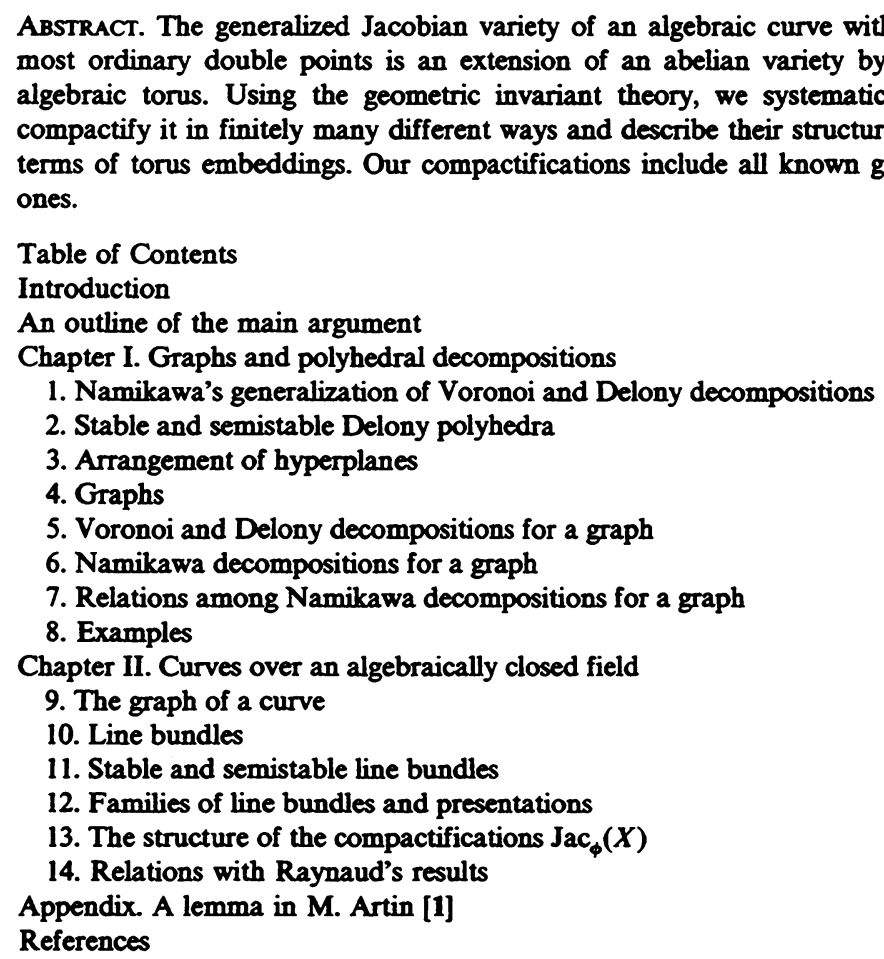

Introduction. Let $X$ be a connected complete curve over an algebraically closed field, which is reduced and has at most ordinary double points. In this

Received by the editors May 17, 1977.

AMS (MOS) subject classifications (1970). Primary 14H40, 14K30, 14K10, 32G13; Secondary 05B45, 05C99, 10E25, 52A25, 32G15.

Key words and phrases. Generalized Jacobian variety, line bundles, Picard scheme, stable curve, geometric invariant theory, torus embedding, Néron model, Delony decomposition, Voronoi decomposition, Namikawa decomposition, arrangement of hyperplanes, spanning tree, complexity of a graph, Kirchhoff-Ttent's theorem, elementary cycle, elementary cocycle.

'The main part of this work was done during the first author's visit to the Tata Institute of Fundamental Research in winter 1973/74. He would like to thank the members of TIFR for their hospitality. 
paper we construct good compactifications of its generalized Jacobian variety $\mathrm{Pic}_{X}^{0}$.

For a compactification $\operatorname{Jac}(X)$ to be good, it ought to satisfy at least the following conditions:

(1) Points of $\operatorname{Jac}(X)$ stand for geometric objects on $X$. In our case, they stand for torsion-free $\theta_{X}$-modules of rank one on each component of $X$.

(2) $\operatorname{Pic}_{X}^{0}$ acts on $\operatorname{Jac}(X)$. In our case, the action corresponds to the tensor product $L \otimes_{\Theta_{X}} F$ of an invertible sheaf $L$ and a torsion-free $\theta_{X}$-module $F$.

(3) $\operatorname{Jac}(X)$ should be proper over $k$. Our compactifications $\operatorname{Jac}_{\phi}(X)$ are reduced projective algebraic schemes over $k$ by its very construction by means of the geometric invariant theory.

(4) When we have a nice family of curves, the compactifications for each fiber should fit into a nice family. Especially

(4') $\operatorname{Jac}(X)$ should be a compactification of the union of a finite number of copies of $\mathrm{Pic}_{X}^{0}$, not just one copy.

For instance, let $Y / S$ be a proper and flat family of curves over the spectrum $S=\{\eta, s\}$ of a discrete valuation ring with $Y_{s}=X$ and the generic fiber $Y_{\eta}$ smooth. The relative Picard functor $\mathrm{Pic}_{Y / S}$ need not be separated nor representable. But Raynaud [29] showed that its greatest separated quotient $Q=Q_{Y / S}$ is represented by a separated and smooth group scheme over $S$. Moreover, $Q^{\tau}$ is the Néron model of the Jacobian variety $\operatorname{Pic}_{Y_{\eta}}^{0}$ of the generic fiber, for example when $Y$ is regular. In this case $\left(Q^{r}\right)_{s}$ contains $\mathrm{Pic}_{X}^{0}$ as a subgroup of finite index. As a matter of fact, this index turns out to be the complexity of the graph of $X$, i.e. the number of spanning trees in the graph, by Kirchhoff-Trent's theorem, as we see in $\$ 14$. In particular, it depends only on $X$ and not on the family $Y / S$ containing $X$. Our $\operatorname{Jac}_{\phi}(X)$ for nondegenerate $\phi$ is a compactification of $\left(Q^{\tau}\right)_{s}$. On the other hand, if $\phi$ is very degenerate, then $\operatorname{Jac}_{\phi}(X)$ is a stable quasi-abelian variety of Namikawa [27] and Nakamura [26], corresponding to the Voronoi decomposition.

Anyhow, we see that

(5) there are many different ways of compactifying the generalized Jacobian variety.

In our case, the difference comes from the distribution of points over the components of $X$ when we use the geometric invariant theory. (5) is not a drawback of the theory but is rather a merit (cf. Mumford [221).

The compactification problem was first studied by Igusa [14] using Chow varieties in connection with a Lefschetz pencil for a surface. The idea of taking torsion-free rank one sheaves for points at infinity of the compactification first occurs in Mumford and Mayer [18], [19]. D'Souza recently carried out the idea when the curve $X$ is irreducible. Nakamura [20] and Namikawa [27] dealt with the problem in the complex analytic context. Here we use the 
same technique as D'Souza's. Namely, our main tool is the basic existence of the good quotient by $\operatorname{PGL}(E)$ of the set of semistable points of a product of projective spaces $\mathbf{P}(E)$ in Mumford [20]. This technique was already applied successfully also to the moduli of vector bundles on a smooth curve by Seshadri [32], and on a surface by Maruyama [16. See also Gieseker [8]. Although we deal with rank one sheaves only, the situation we encounter turns out to be quite similar to the case of vector bundles over a smooth curve, the number of components of $X$ playing somewhat the role of the rank of vector bundles.

It should be mentioned here that our method does not work for nonreduced curves, or a family of curves with multiple fibers. To deal with this case, we need to consider the quotient of the Hilbert scheme of 0 -cycles of a projective space, instead of a product of projective spaces. But our case is enough for many applications, notably the moduli of stable curves.

We now state our main results.

The combinatorial configuration of the irreducible components of $X$ and their intersections at double points very much affect our considerations. We express this configuration, as usual, by the graph $\Gamma(X)$ of $X$ ( $\$ 4$ and 9$)$. The genus of the normalization of each component of $X$ does not much affect our combinatorial considerations. Fixing an orientation for $\Gamma(X)$, we can define chain groups $C_{0}(\Gamma(X), \mathbf{Z}), C_{1}(\Gamma(X), \mathbf{Z})$, the first homology and cohomology groups $H_{1}(\Gamma(X), \mathbf{Z}), H^{1}(\Gamma(X), \mathbf{Z})$, the 0-boundary group $\partial C_{1}(\Gamma(X), \mathbf{Z})$ and the 1-coboundary group $\delta C_{0}(\Gamma(X), Z)$. For simplicity, let us omit $\Gamma(X)$ from these notations, and let us denote by $C_{0}(\mathbf{R})$ etc. the corresponding scalar extensions to the real number field $\mathbf{R}$. We denote by $\rho: C^{1}=C_{1} \rightarrow H^{1}$ the canonical projection.

(I) (PRoposition 6.1) To each $\phi \in \partial C_{1}(\mathbf{R})$, there corresponds a polyhedral decomposition $\mathrm{Del}_{\phi}$ of $H^{1}(\mathbf{R})$, called the Namikawa decomposition, consisting of bounded polyhedra such that

(i) $\mathrm{Del}_{\phi}$ is invariant under the translation action of $\rho\left(H_{1}(\mathbf{Z})\right)$ with the quotient cell complex $\overline{\operatorname{Del}}_{\phi}=\operatorname{Del}_{\phi} / \rho\left(H_{1}(Z)\right)$ finite. This quotient is called the Namikawa cell complex.

(ii) The set of 0-dimensional polyhedra, the 0-skeleton, satisfies

$$
\mathrm{Sk}^{0}\left(\operatorname{Del}_{\phi}\right) \subset H^{1}(\mathrm{Z})
$$

These decompositions come in naturally from the geometric invariant theory. But it was Namikawa who pointed out that they are obtained as slight generalizations of the Delony decomposition developed in $\$ 1$, applied to the graph $\Gamma(X)$ in $\S 6$.

The Namikawa decompositions for a different $\phi$ can be compared as follows:

(II) (Proposition 2.3 and Theorem 7.1) There exists a polyhedral decom- 
position Arr of $\partial C_{1}(\mathbf{R})$, called an arrangement of hyperplanes, consisting of bounded polyhedra such that

(i) Arr is invariant under the translation action of $\partial C_{1}(\mathbf{Z})$ with the quotient finite, and

(ii) $\mathrm{Sk}^{0}$ (Arr) $\supset \partial C_{1}(\mathbf{Z})$.

(iii) (Corollary 7.2) Given a polyhedron $A \in$ Arr, the Namikawa decomposition Del $\phi$ remains constant as long as $\phi-\partial e(J) / 2$ stays in $\operatorname{rel}$.int $(A)+$ $\partial \delta C_{0}(\mathrm{Z})$ where $e(J) \in C_{1}(Z)$ is canonically defined by the graph. Especially, the number of different Namikawa decompositions is finite, namely the cardinality of $\mathrm{Arr} / \partial \delta C_{0}(\mathrm{Z})$. Even among them, there are the following relations:

(iv) (Corollary 7.2) Let $\bar{A}$ be a face of $A \in$ Arr. For $\bar{\phi}-\partial e(J) / 2 \in$ $\operatorname{rel}$.int $(\bar{A})$ and $\phi-\partial e(J) / 2 \in \operatorname{rel}$.int $(A)$, we see that $\operatorname{Del}_{\phi}$ is a subdivision of $\operatorname{Del}_{\bar{\phi}}$.

(v) (Corollary 7.2) If $A^{\prime}$ is the translation of $A \in$ Arr by an element of $\partial C_{1}(\mathrm{Z})$; then $\operatorname{Del}_{\phi^{\prime}}$ for $\phi^{\prime}-\partial e(J) / 2 \in \operatorname{rel}$.int $\left(A^{\prime}\right)$ is the translation of $\operatorname{Del}_{\phi}$ for $\phi-\partial e(J) / 2 \in \operatorname{rel}$.int $(A)$ by an element of $H^{1}(\mathbf{Z})$.

(vi) (Theorem 7.7) $\mathrm{Sk}^{0}\left(\operatorname{Del}_{\phi}\right)=H^{1}(\mathrm{Z})$ holds if $\phi-\partial e(J) / 2$ lies in the relative interior of a top-dimensional polyhedron in Arr. We call such $\phi$ nondegenerate. The corresponding Namikawa decomposition is the finest.

(vii) (Proposition 7.8) If $\phi-\partial e(J) / 2$ is a 0-dimensional polyhedron belonging to $\partial C_{1}(\mathbf{Z})$, then $\mathrm{Del}_{\phi}$ is essentially the Voronoi decomposition of $H_{1}(\mathbf{R})$ with respect to $H_{1}(\mathrm{Z})$. It is the coarsest Namikawa decomposition. But in general, there are other Namikawa decompositions which are the coarsest, since we need not have the equality in (IIii) (cf. \$8, Example (5)).

Let $X=\cup_{i \in I} X_{i}$ be the decomposition of $X$ into irreducible components and $\left\{Q_{j}\right\}_{j \in J}$ the set of double points of $X$. Thus $I$ and $J$ are the vertex set and the edge set of the graph $\Gamma(X)$, respectively. For subset $J^{\prime} \subset J$, let $X\left(J^{\prime}\right)$ be the partial normalization of $X$ obtained by the blowing up of $X$ along $\left\{Q_{j}\right\}_{j \in J-J}$. In particular, $\tilde{X}=X(\varnothing)$ is the normalization of $X$, and $X(J)=$ $X$.

There exist canonical surjections (cf. §10)

$$
\text { deg: } \operatorname{Pic}_{\tilde{X}} \rightarrow C_{0}(\mathbf{Z}), \quad \mathcal{L}: \mathrm{Pic}_{X\left(J^{\prime}\right)} \rightarrow \mathrm{Pic}_{\tilde{X}} \text {. }
$$

For $m \in C_{0}(\mathbf{Z})$, let $\operatorname{Pic}_{X\left(J^{\prime}\right)}^{m}$ be the inverse image of $m$ by the composition deg $\circ$ L.

(III) (i) (Corollary 12.4) $\mathrm{Pic}_{x}^{m}$ is a principal $T$-bundle over $\mathrm{Pic}_{\tilde{X}}^{m}$, where $T=G_{m} \otimes H^{1}(\mathrm{Z})$ is the algebraic torus over $k$ whose character group is $H_{1}(\mathrm{Z})$.

For $\bar{N} \in \overline{\mathrm{Del}}_{\phi}$, we can define canonically an element $m(\bar{N}) \in C_{0}(\mathbf{Z})$ and a subset Supp $\bar{N} \subset J$. On the other hand, for each $\bar{\xi} \in \mathrm{Sk}^{0}\left(\overline{\mathrm{Del}}_{\phi}\right)$, we can define a complete torus embedding (cf. §13) 


$$
T \subset \operatorname{Temb}(\bar{\xi}) \text {. }
$$

Consider the fiber bundle

$$
\operatorname{Pic}_{X}^{m(\xi)} \times{ }^{T} \operatorname{Temb}(\bar{\xi})
$$

associated with the principal $T$-bundle $\operatorname{Pic}_{X}^{m(\xi)}$ over $\operatorname{Pic}_{\bar{X}}^{m(\xi)}$ and the $T$-action on $\operatorname{Temb}(\bar{\xi})$.

(ii) (Theorem 11.4 and Proposition 12.17) Given $\phi \in \partial C_{1}(\mathbf{R})$, there exists a reduced projective algebraic scheme $\operatorname{Jac}_{\phi}(X)$ with an action of $\operatorname{Pic}_{X}^{0}$ and a bijection

$$
\text { O: } \overline{\operatorname{Del}}_{\phi} \stackrel{\sim}{\rightarrow}\left\{\text { Pic }_{X}^{0} \text {-orbits in } \mathrm{Jac}_{\phi}(X)\right\}
$$

which is order reversing, i.e. $\bar{N}^{\prime}$ is a face of $\bar{N}$ if and only if $\mathbf{O}(\bar{N})$ is in the closure of $\mathbf{O}\left(\bar{N}^{\prime}\right)$.

(iii) (Theorem 13.2) The normalization of $\operatorname{Jac}_{\phi}(X)$ is the disjoint union of $\operatorname{Pic}_{X}^{m(\bar{\xi})} \times{ }^{T} \operatorname{Temb}(\bar{\xi})$ with $\bar{\xi}$ running over $\operatorname{Sk}^{0}\left(\operatorname{Del}_{\phi}\right)$. For $N \in \operatorname{Del}_{\phi}$ and a vertex $\xi$ of $N$, let $\bar{N}$ and $\bar{\xi}$ be their respective image in $\overline{\mathrm{Del}}_{\phi}$. Then there exists an imbedding

$$
g_{\xi}(N): \operatorname{Pic}_{X(J-S u p p}^{m(\bar{N})} \hookrightarrow \operatorname{Pic}_{X}^{m(\xi)} \times^{T} \operatorname{Temb}(\bar{\xi})
$$

such that its composition with the projection onto $\operatorname{Jac}_{\phi}(X)$ induces an isomorphism

$$
\operatorname{Pic}_{X(J-\operatorname{Supp}}^{m(\bar{N})} \stackrel{\sim}{\rightarrow} \mathbf{O}(\bar{N}) \subset \mathrm{Jac}_{\phi}(X) .
$$

$\mathrm{Jac}_{\phi}(X)$ is obtained from the normalization by means of the identifications induced by the morphisms $g_{\xi}(N)$ with $N$ running over $\operatorname{Del}_{\phi}$ and $\xi$ over its vertices.

$\mathrm{Jac}_{\phi}(X)$ is first shown to exist as a good quotient of a Hilbert scheme by means of the geometric invariant theory (Theorem 11.4). $\mathrm{Jac}_{\phi}(X)$ is the coarse moduli of $\phi$-equivalence classes of $\phi$-semistable line bundles on $X$, and its open set $\operatorname{Jac}_{\phi \text {-stable }}(X)$ is the fine moduli of $\phi$-stable line bundles (Theorem 12.4). Here we mean by a line bundle on $X$ a torsion-free coherent $\theta_{X}$-module which is of rank one on each component. Unlike the case of vector bundles, these notions of semistability and stability are not intrinsic properties of line bundles. In particular, when $\phi$ is nondegenerate, then $\phi$-semistability and $\phi$-stability coincide, hence $\operatorname{Jac}_{\phi}(X)$ is the fine moduli.

Thus $\operatorname{Jac}_{\phi}(X)$ satisfies our requirements (1), (2), (3) and (5). (4') is shown in \$14. The proof of (4) was carried out by Ishida [36].

We see in the last example of $\$ 13$ that when $X$ is a stable curve of genus 2 , for instance, then $\mathrm{Jac}_{\phi}(X)$ 's we obtain coincide with those in Mumford [22], Namikawa [27] and Nakamura [26].

The key to connecting (I) and (III) above is Theorem 10.5, which shows 
that there exists a canonical bijection $\bar{D}$ from the set of $\operatorname{Pic}^{0}(X)$-orbits in $\mathrm{LB}^{0}(X)=\left\{\right.$ line bundles $F$ on $X$ with $\left.\chi(F)=\chi\left(\theta_{X}\right)\right\}$ to the set $\bar{K}(\Gamma(X))$ of $H_{1}(\mathbf{Z})$-equivalence classes of Delony polyhedra in $C_{1}(\mathbf{R}) . \bar{D}$ has many nice properties and is a generalization to line bundles of the notion of degree for invertible sheaves.

An outline of the main argument. We now give a brief outline of that part of our argument which uses the geometric invariant theory. We actually follow the argument in reverse here. We will then be able to see the motivation for various concepts introduced in this paper. Especially the reason (5) why we have many different compactifications and $\left(4^{\prime}\right)$ why they contain several copies of the generalized Jacobian variety will become apparent.

For simplicity, we assume here that the curve $X=\bigcup_{i \in I} X_{i}$ has nonsingular irreducible components $X_{i}$. Let $\left\{Q_{j}\right\}_{j \in J}$ be the set of double points of $X$. Thus $I$ and $J$ are the vertex set and the edge set of the graph $\Gamma(X)$, respectively. An edge $j \in J$ joins vertices $i$ and $i^{\prime}$ if $Q_{j}$ is an intersection of $X_{i}$ and $X_{i^{\prime}}$ ' The graph $\Gamma(X)$ in this case has no loops. Let us denote $\chi_{i}=\chi\left(\theta_{X_{i}}\right)$.

Given an invertible sheaf $F$ on $X$, let $F_{i}$ be the restriction of $F$ to $X_{i}$. Then we obviously have an exact sequence

$$
0 \rightarrow F \rightarrow \bigoplus_{i \in I} F_{i} \rightarrow \bigoplus_{j \in J} k\left(Q_{j}\right) \rightarrow 0
$$

where $k\left(Q_{j}\right)$ is the sheaf on $X$ concentrated at $Q_{j}$ with $k$ as the stalk. If $n_{i}$ is the degree of $F_{i}$ as an invertible sheaf on $X_{i}$, then we have

$$
\chi(F)=n(I)+\chi(I)-|J|,
$$

where $|J|$ is the cardinality of $J$, and for a subset $I^{\prime}$ of $I$, we denote $n\left(I^{\prime}\right)=\sum_{i \in I^{\prime}} n_{i}$ and $\chi\left(I^{\prime}\right)=\sum_{i \in I^{\prime}} \chi_{i}$.

Given big enough integers $\left\{\tilde{n}_{i}\right\}_{i \in I}$, consider a $k$-vector space $E$ with $\operatorname{dim} E=\tilde{n}(I)+\chi(I)-|J|$. Let $Q(E / P)=\operatorname{Quot}\left(\theta_{X} \otimes_{k} E / P\right)$ be Grothendieck's Hilbert scheme parametrizing coherent quotient $\theta_{X}$-modules $G_{q}$ of $\theta_{X} \otimes_{k} E$ which is of rank one on each component $X_{i}$ and with $\chi\left(G_{q}\right)=\tilde{n}(I)+\chi(I)-|J|$. Consider its open subset $R(E / P)$ consisting of points $q$ such that

$$
\left\{\begin{array}{l}
G_{q} \text { is } \Theta_{X} \text {-torsion free, } \\
H^{1}\left(G_{q}\right)=0, \\
E \rightarrow H^{0}\left(G_{q}\right) \text { an isomorphism. }
\end{array}\right.
$$

If $F$ is an invertible sheaf on $X$ whose degrees $n_{i}$ are sufficiently close to $\tilde{n}_{i}$ and $n(I)=\tilde{n}(I)$, there exists a $G L(E)$-orbit in $R(E / P)$ such that $F \cong G_{q}$ if and only if $q$ belongs to the orbit.

Thus the quotient of $R(E / P)$ by $\mathrm{GL}(E)$, if it exists, would parametrize isomorphism classes of certain torsion-free $\theta$-modules, and contains the set 
of isomorphism classes of invertible sheaves $F$ whose degrees $n_{i}$ are sufficiently close to $\tilde{n}_{i}$ with $n(I)=\tilde{n}(I)$. Unfortunately, such a quotient does not exist as a scheme. We now try to find a suitable open set of $R(E / P)$ for which the quotient exists.

Consider nonsingular points $x_{1}, \ldots, x_{N}$ of $X$. They give rise to a $\mathrm{GL}(E)$ equivariant morphism

$$
\tau: R(E / P) \rightarrow \mathbf{P}(E) \times \mathbf{P}(E) \times \cdots \times \mathbf{P}(E)=Z
$$

which sends $q$ to $\tau(q)=\left(\tau_{1}(q), \ldots, \tau_{N}(q)\right)$, where $\tau_{\alpha}(q): E \rightarrow-G_{q}\left(x_{\alpha}\right)=$ (the fiber of $G_{q}$ at $x_{\alpha}$ ) is the corresponding 1-dimensional quotient. If $N$ and $\left\{\tilde{n}_{i}\right\}_{i \in I}$ are large enough and properly chosen, then $\tau$ is injective.

Let $z=\left(z_{1}, \ldots, z_{N}\right)$ be a point of $Z$. Each $z_{\alpha}: E \rightarrow E_{\alpha}$ is thus a 1-dimensional quotient. The point $z$ is called semistable (resp. stable) if for any nonzero proper subspace $E^{\prime}$ of $E$, we have

$$
\sum_{1<\alpha<N} \operatorname{dim} z_{\alpha}\left(E^{\prime}\right) / N \geqslant \operatorname{dim} E^{\prime} / \operatorname{dim} E \quad \text { (resp. }>\text { ). }
$$

It is one of the basic results in the geometric invariant theory that the set $Z^{s s}$ (resp. $Z^{s}$ ) of semistable (resp. stable) points have a good projective (resp. quasi-projective geometric) quotient $Z^{s s} / \mathrm{GL}(E)$ (resp. $Z^{s} / \mathrm{GL}(E)$ ).

Let us call $q \in R(E / P)$ and $G_{q}$ semistable (resp. stable) if $\tau(q)$ is semistable (resp. stable). Then the set $R(E / P)^{s s}$ of semistable points have a projective good quotient $R(E / P)^{s s} / \mathrm{GL}(E)$. For the quotient to be a compactification we are looking for, all the points $q$ with $G_{q}$ invertible and with fixed degrees $n_{i}$ should be semistable.

For $q$ to be semistable (resp. stable), $G_{q}$ should staisfy

$$
\sum_{1<\alpha<N} \operatorname{dim} \tau_{\alpha}(q)\left(E^{\prime}\right) / N \geqslant \operatorname{dim} E^{\prime} / \operatorname{dim} E \quad \text { (resp. }>\text { ) }
$$

for any nonzero proper subspace $E^{\prime}$ of $E$. It turns out that if $N$ and $\left\{\tilde{n}_{i}\right\}_{i \in I}$ are large enough and properly chosen, then these inequalities (*) suffice to hold only for subspaces $E^{\prime}=E_{I^{\prime}}$ for any nonempty proper subsets $I^{\prime}$ of $I$, where $E_{I^{\prime}}$ is the subspace of $E$ consisting of sections which vanish completely on the subcurve $\cup_{i \in I^{\prime}} X_{i}$.

Let $G_{q}$ be invertible with $n_{i}$ the degree of its restriction to $X_{i}$. Thus $\operatorname{dim} E=\chi\left(G_{q}\right)=n(I)+\chi(I)-|J|$ implies that $n(I)=\tilde{n}(I)$. It is not hard to see that $\operatorname{dim} E_{I^{\prime}}=n\left(I-I^{\prime}\right)+\chi\left(I-I^{\prime}\right)-\mid\{j \in J$; at least one end of $j$ is in $\left.I-I^{\prime}\right\} \mid$. On the other hand, $\operatorname{dim} \tau_{\alpha}(q)\left(E_{I^{\prime}}\right)=0$ or 1 , according as $x_{\alpha}$ is on $\cup_{i \in I^{\prime}} X_{i}$ or not. Let $N_{i}$ be the number of points $x_{\alpha}$ which are on $X_{i}$. Then the inequality (*) for $E^{\prime}=E_{I-I}$, becomes

$N\left(I^{\prime}\right) / N \geqslant\left[n\left(I^{\prime}\right)+\chi\left(I^{\prime}\right)-\mid\{j \in J ;\right.$ at least one end of

$$
\left.\left.j \text { is in } I^{\prime}\right\} \mid\right] /\{\tilde{n}(I)+\chi(I)-|J|\} .(* *)
$$


Let $\lambda_{i}=N_{i} / N$. Then $\lambda(I)=1$. Note that the inequality $(* *)$ depends not on $N$ but only on the distribution ratio $\left\{\lambda_{i}\right\}_{i \in I}$ of points over the components of $X$. To make the inequalities (**) look simpler, we normalize it as follows: Let $d_{i}$ be the number of edges $j \in J$ one of whose end is $i$. In graph theory, $d_{i}$ is called the degree of the vertex $i$. There is a basic equality $d(I)=2|J|$. Let us introduce rational numbers $\phi_{i}$ defined by

$$
\lambda_{i}=\left\{\tilde{n}_{i}+\chi_{i}-d_{i} / 2+\phi_{i}\right\} /\{\tilde{n}(I)+\chi(I)-|J|\} .
$$

We see that $\lambda(I)=1$ is equivalent to $\phi(I)=0$. Since $n(I)=\tilde{n}(I),(* *)$ can be rewritten as

$$
n\left(I^{\prime}\right)-\tilde{n}\left(I^{\prime}\right) \leqslant \phi\left(I^{\prime}\right)+(1 / 2) \mid\left\{j \in J ; j \text { joins } I^{\prime} \text { and } I-I^{\prime}\right\} \mid \text {. }
$$

We thus conclude the following: Let $\left\{\phi_{i}\right\}_{i \in I}$ be fixed with $\phi(I)=0$, i.e. we fix the distribution ratio of points over the components of $X$. Let $q$ be a point of $R(E / P)$ such that $G_{q}$ is an invertible sheaf whose restriction to $X_{i}$ has degree $n_{i}$. In particular, we have $n(I)=\tilde{n}(I)$. Then $q$ is semistable (resp. stable) if and only if (***) is satisfied for any nonempty proper subset $I^{\prime}$ of $I$ (resp. strict inequalities). In general, there are only a finite number of integral solutions $\left\{n_{i}\right\}_{i \in I}$. Thus they are always sufficiently close to $\left\{\tilde{n}_{i}\right\}_{i \in I^{\circ}}$.

For simplicity, let us consider the case $|I|=2$ and $|J|=3$, i.e. $X$ looks like the "dollar sign" (see Figure 1).
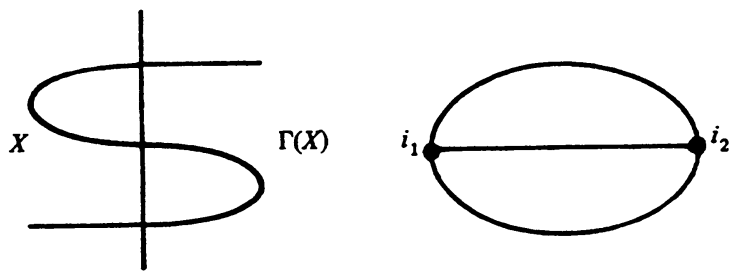

FIGURE 1

For $\left\{\phi_{1}, \phi_{2}\right\}$ with $\phi_{1}+\phi_{2}=0$ fixed, our requirements are $n(I)=\tilde{n}(I)$ and $(* * *)$ for any $I^{\prime}$, i.e.

$$
\begin{gathered}
\left(n_{1}-\tilde{n}_{1}\right)+\left(n_{2}-\tilde{n}_{2}\right)=0 \\
\left(n_{1}-\tilde{n}_{1}\right) \leqslant \phi_{1}+(3 / 2), \quad\left(n_{2}-\tilde{n}_{2}\right) \leqslant \phi_{2}+(3 / 2),
\end{gathered}
$$

which reduce to

$$
\left(n_{2}-\tilde{n}_{2}\right)=-\left(n_{1}-\tilde{n}_{1}\right), \quad \phi_{1}-(3 / 2) \leqslant\left(n_{1}-\tilde{n}_{1}\right)<\phi_{1}+(3 / 2) .
$$

(a) If $\phi_{1}=0$, then the integral solutions are $\left(n_{1}, n_{2}\right)=\left(\tilde{n}_{1}+1, \tilde{n}_{2}-1\right)$, $\left(\tilde{n}_{1}, \tilde{n}_{2}\right)$ and $\left(\tilde{n}_{1}-1, \tilde{n}_{2}+1\right)$. Moreover, strict inequalities are satisfied for all these solutions. Thus they correspond to stable invertible sheaves. In this case, we conclude that three copies of the generalized Jacobian variety Pic $_{X}^{0}$ are contained in the compactification $R(E / P)^{s s} / G L(E)$. 
(b) If $\phi_{1}=(1 / 2)$, then the integral solutions are $\left(n_{1}, n_{2}\right)=\left(\tilde{n}_{1}+1, \tilde{n}_{2}-1\right)$, $\left(\tilde{n}_{1}, \tilde{n}_{2}\right),\left(\tilde{n}_{1}+2, \tilde{n}_{2}-2\right)$ and $\left(\tilde{n}_{1}-1, \tilde{n}_{2}+1\right)$. Moreover, the strict inequalities are satisfied for the first two solutions. Thus they correspond to stable invertible sheaves. As we see in the examples of $\S 13, R(E / P)^{s s} / \mathrm{GL}(E)$ in this case contains only two copies of $\operatorname{Pic}_{X}^{0}$ corresponding to the first two solutions.

\section{CHAPTER I. GRAPHS AND POLYHEDRAL DECOMPOSITIONS}

1. Namikawa's generalization of Voronoi and Delony decompositions. We first recall Voronoi and Delony decompositions. See Rogers [30] and Voronoi [34].

Let $E$ be a finite-dimensional real vector space with a positive definite symmetric bilinear form $(x, y)$ on $E$. We denote by $\|x\|=(x, x)^{1 / 2}$ the associated norm on $E$. Let $\Lambda$ be a Z-lattice in $E$.

It is a well-known fact that for $\xi$ in $\Lambda$ the set

$$
V(\xi)=\{x \in E ;\|x-\xi\| \leqslant\|x-\eta\| \text { for all } \eta \text { in } \Lambda\}
$$

is a bounded polyhedron, and is a fundamental domain of $E$ with respect to the translation action of $\Lambda$. Moreover we have $V\left(\xi+\xi^{\prime}\right)=V(\xi)+\xi^{\prime}$ for all $\xi^{\prime}$ in $\Lambda$.

The following is an immediate consequence of this observation:

Proposition 1.1. The set $\operatorname{Vor}(E, \Lambda)$ of Voronoi polyhedra in $E$ consisting of $V(\xi)$ for $\xi$ in $\Lambda$ and their faces constitute a polyhedral decomposition, called the Voronoi decomposition, of $E$ by bounded polyhedra. Moreover, $\operatorname{Vor}(E, \Lambda)$ is invariant under the translation action of $\Lambda$ with $\operatorname{Vor}(E, \Lambda) / \Lambda$ finite. $A$ Voronoi polyhedron $V$ is of the following form: there exist $\xi_{0}, \ldots, \xi_{r}$ in $\Lambda$ such that the relative interior of $V$ is of the form

$$
\begin{aligned}
\left\{x \in E \mid\left\|x-\xi_{0}\right\|=\cdots=\left\|x-\xi_{r}\right\|\right. & <\|x-\eta\|, \\
\Lambda & \left.\ni \forall \eta \neq \xi_{0}, \ldots, \xi_{r}\right\}
\end{aligned}
$$

and

$$
V=\left\{x \in E \mid\left\|x-\xi_{0}\right\|=\cdots=\left\|x-\xi_{r}\right\| \leqslant\|x-\eta\| \text { for all } \eta \text { in } \Lambda\right\} .
$$

For $x$ in $E$ consider the convex hull

$$
D(x)=\left\langle\xi_{0}, \ldots, \xi_{r}\right\rangle=\left\{\sum a_{i} \xi_{i} ; a_{i}>0, \sum a_{i}=1\right\}
$$

where $\left\{\xi_{0}, \ldots, \xi_{r}\right\}$ is the set of $\xi$ in $\Lambda$ for which $\|x-\xi\|$ attains the smallest value.

Definimion. We denote by $\operatorname{Del}(E, \Lambda)$ the set of polyhedra $D$ in $E$ of the form $D=D(x)$ for some $x$ in $E$. We call such $D$ a Delony polyhedron.

We have easily the following:

LEMMA 1.2. Given a Delony polyhedron $D$, there exists a unique Voronoi polyhedron $V$ such that $D=D(x)$ if and only if $x$ is in the relative interior of $V$. We denote $V=D^{*}, D=V^{*}$ and call $V$ and $D$ dual to each other. 
Proposition 1.3. $\operatorname{Del}(E, \Lambda)$ is a polyhedral decomposition, called the Delony decomposition, of $E$ by bounded polyhedra with the set $\operatorname{Sk}^{0}(\operatorname{Del}(E, \Lambda))$ of 0-dimensional polyhedra coinciding with $\Lambda$. $\operatorname{Del}(E, \Lambda)$ is invariant under the translation action of $\Lambda$ with $\operatorname{Del}(E, \Lambda) / \Lambda$ finite. Moreover, $\operatorname{Vor}(E, \Lambda)$ and $\operatorname{Del}(E, \Lambda)$ are decompositions of $E$ dual to each other in the sense that

(i) $V_{2}$ is a face of $V_{1}$ if and only if $V_{1}^{*}$ is a face of. $V_{2}^{*}$ for $V_{1}$ and $V_{2}$ in $\operatorname{Vor}(E, \Lambda)$, and

(ii) $\operatorname{dim} V+\operatorname{dim} V^{*}=\operatorname{dim} E$ for $V$ in $\operatorname{Vor}(E, \Lambda)$.

This proposition is a special case $E^{\prime}=E$ of the following generalization, the relevance of which was pointed out to us by Y. Namikawa. It helped to clarify considerably our original version of the polyhedral decompositions associated to our compactification of the generalized Jacobian variety.

Let $E$ and $\Lambda$ be as before. Consider a subspace $E^{\prime}$ of $E$ such that $\Lambda \cap E^{\prime}$ is a Z-lattice in $E^{\prime}$. We denote by $E^{\prime \prime}$ the orthogonal complement of $E^{\prime}$ in $E$. Let $\pi^{\prime}: E \rightarrow E^{\prime}$ and $\pi^{\prime \prime}: E \rightarrow E^{\prime \prime}$ be the orthogonal projections. Suppose further that $\pi^{\prime}(\Lambda)$ is a lattice in $E^{\prime}$, or equivalently that $\Lambda \cap E^{\prime \prime}$ is a lattice in $E^{\prime \prime}$. Then obviously $\pi^{\prime}(\Lambda)$ is a lattice in $E^{\prime}$ containing $\Lambda \cap E^{\prime}$ and $\pi^{\prime \prime}(\Lambda)$ is a lattice in $E^{\prime \prime}$ containing $\Lambda \cap E^{\prime \prime}$.

For $\psi$ in $E^{\prime \prime}$ consider the coset $E_{\psi}^{\prime}=E^{\prime}+\psi$. Then we obviously have the following:

Proposirion 1.4. The set of polyhedra $\operatorname{Vor}\left(E_{\psi}^{\prime} ; E, \Lambda\right)$ consisting of $V^{\prime}=V$ $\cap E_{\psi}^{\prime}$ with $V$ running over all the Voronoi polyhedra of $E$ such that $\operatorname{rel}$ int $(V)$ $\cap E_{\psi}^{\prime}=\varnothing$, is a polyhedral decomposition, called the induced Voronoi decomposition, of $E_{\psi}^{\prime}$. It is invariant under the translation by $\Lambda \cap E^{\prime}$ with $\operatorname{Vor}\left(E_{\psi}^{\prime}\right.$; $E, \Lambda) / \Lambda \cap E^{\prime}$ finite.

Definition. We denote by $\operatorname{Del}_{\psi}\left(E^{\prime}: E, \Lambda\right)$ the set of polyhedra $D^{\prime}$ in $E^{\prime}$ of the form $D^{\prime}=\pi^{\prime}(D(x))$ for $x$ in $E_{\psi}^{\prime}$.

Then as before we have the following:

LemMa 1.5. For $D^{\prime}$ in $\operatorname{Del}_{\psi}\left(E^{\prime} ; E, \Lambda\right)$ there exists a unique $V^{\prime}$ in $\operatorname{Vor}\left(E_{\psi}^{\prime}\right.$; $E, \Lambda)$ such that for $x$ in $E_{\psi}^{\prime}$ we have $D^{\prime}=\pi^{\prime}(D(x))$ if and only if $x$ is in the relative interior of $V^{\prime}$. We denote $D^{\prime}=\left(V^{\prime}\right)^{*}, V^{\prime}=\left(D^{\prime}\right)^{*}$ and call them dual to each other.

Proposition 1.6. $\operatorname{Del}_{\psi}\left(E^{\prime} ; E, \Lambda\right)$ is a polyhedral decomposition, called the Namikawa decomposition, of $E^{\prime}$ by bounded polyhedra invariant under the translation action of the lattice $\Lambda \cap E^{\prime}$ with $\operatorname{Del}_{\psi}\left(E^{\prime} ; E, \Lambda\right) / \Lambda \cap E^{\prime}$ finite. Moreover,

(i) $\operatorname{dim} D^{\prime}+\operatorname{dim}\left(D^{\prime}\right)^{*}=\operatorname{dim} E^{\prime}$ for $D^{\prime}$ in $\operatorname{Del}_{\psi}\left(E^{\prime} ; E, \Lambda\right)$,

(ii) the set of 0-dimensional polyhedra $\operatorname{Sk}^{0}\left(\operatorname{Del}_{\psi}\left(E^{\prime} ; E, \Lambda\right)\right)$ is a subset of the lattice $\pi^{\prime}(\Lambda)$, and 
(iii) $D_{1}^{\prime}$ is a face of $D_{2}^{\prime}$ if and only if $\left(D_{2}^{\prime}\right)^{*}$ is a face of $\left(D_{1}^{\prime}\right)^{*}$ for $D_{1}^{\prime}$ and $D_{2}^{\prime}$ in $\operatorname{Del}_{\psi}\left(E^{\prime} ; E, \Lambda\right)$.

Proof. We prove this, for completeness, exactly as in Rogers [30]. The invariance of $\operatorname{Del}_{\psi}\left(E^{\prime} ; E, \Lambda\right)$ under $\Lambda \cap E^{\prime}$ and the finiteness of the quotient follows easily from the corresponding result for $\operatorname{Vor}\left(E_{\psi}^{\prime} ; E, \Lambda\right)$ and the previous lemma.

(ii) is obvious.

(1) We next show (i). Let $D^{\prime}=\left(V^{\prime}\right)^{*}$ with

$$
\begin{array}{r}
\operatorname{rel.int}\left(V^{\prime}\right)=\left\{x \in E_{\psi}^{\prime} \mid\left\|x-\xi_{0}\right\|=\cdots=\left\|x-\xi_{r}\right\|<\|x-\eta\|\right. \\
\text { for all } \left.\eta \text { in } \Lambda \neq \xi_{0}, \ldots, \xi_{r}\right\} .
\end{array}
$$

These defining relations can be rewritten as

$$
2\left(\eta-\xi_{0}, x\right) \leqslant\|\eta\|^{2}-\left\|\xi_{0}\right\|^{2}
$$

for all $\eta$ in $\Lambda$ with the equality holding if and only if $\eta=\xi_{0}, \ldots, \xi_{r}$. Since the relative interior of $V^{\prime}$ is nonempty by assumption, we see that its dimension is equal to that of the linear space

$$
\left\{y \in E^{\prime} ; 2\left(\xi_{i}-\xi_{0}, y+\psi\right)=\left\|\xi_{i}\right\|^{2}-\left\|\xi_{0}\right\|^{2}, i=1, \ldots, r\right\}
$$

which is obviously equal to the codimension in $E^{\prime}$ of the subspace in $E^{\prime}$ generated by $\pi^{\prime}\left(\xi_{i}-\xi_{0}\right)$ for $i=1, \ldots, r$. This is exactly the codimension of the convex hull $D^{\prime}=\left\langle\pi^{\prime}\left(\xi_{0}\right), \ldots, \pi^{\prime}\left(\xi_{r}\right)\right\rangle$.

(2) We next show that $V_{1}^{\prime}>V_{2}^{\prime}$ implies $\left(V_{1}^{\prime}\right)^{*}<\left(V_{2}^{\prime}\right)^{*}$ for $V_{1}^{\prime}$ and $V_{2}^{\prime}$ in $\operatorname{Vor}\left(E_{\psi}^{\prime} ; E, \Lambda\right)$. Let the relative interior of $V_{1}^{\prime}$ be of the form

$$
\begin{array}{r}
\left\{x \in E_{\psi}^{\prime} \mid\left\|x-\xi_{0}\right\|=\cdots=\left\|x-\xi_{r}\right\|<\|x-\eta\|\right. \\
\text { for all } \left.\eta \text { in } \Lambda \neq \xi_{0}, \ldots, \xi_{r}\right\}
\end{array}
$$

hence $\left(V_{1}^{\prime}\right)^{*}=\left\langle\pi^{\prime}\left(\xi_{0}\right), \ldots, \pi^{\prime}\left(\xi_{r}\right)\right\rangle$. Since $V_{2}^{\prime}$ is a face of $V_{1}^{\prime}$, the relative interior of $V_{2}^{\prime}$ is of the form

$$
\begin{array}{r}
\left\{y \in E_{\psi}^{\prime} \mid\left\|y-\xi_{0}\right\|=\cdots=\left\|y-\xi_{s}\right\|<\|y-\eta\|\right. \\
\left.\quad \text { for all } \eta \text { in } \Lambda \neq \xi_{0}, \ldots, \xi_{s}\right\}
\end{array}
$$

with $s \geqslant r$. Thus for $x$ and $y$ in the respective relative interior of $V_{1}^{\prime}$ and $V_{2}^{\prime}$ we see that

$$
\left(\xi_{i}, y-x\right) \geqslant\left(\xi_{0}, y-x\right)
$$

for $0 \leqslant i \leqslant s$ with the equality holding if and only if $0 \leqslant i \leqslant r$. Thus the hyperplane

$$
H^{\prime}=\left\{z \in E^{\prime} ;(z, y-x)=\left(\xi_{0}, y-x\right)\right\}
$$

has the property that $\left(V_{2}^{\prime}\right)^{*}=\left\langle\pi^{\prime}\left(\xi_{0}\right), \ldots, \pi^{\prime}\left(\xi_{s}\right)\right\rangle$ is contained in its nonnegative side and that

$$
\left(V_{1}^{\prime}\right)^{*}=\left\langle\pi^{\prime}\left(\xi_{0}\right), \ldots, \pi^{\prime}\left(\xi_{r}\right)\right\rangle=H^{\prime} \cap\left(V_{2}^{\prime}\right)^{*}
$$


(3) We now show that for $V_{2}^{\prime}$ in $\operatorname{Vor}\left(E_{\psi}^{\prime} ; E, \Lambda\right)$ and a face $D_{1}^{\prime}$ of $\left(V_{2}^{\prime}\right)^{*}$, there exists $V_{1}^{\prime}>V_{2}^{\prime}$ such that $D_{1}^{\prime}=\left(V_{1}^{\prime}\right)^{*}$. Indeed, let $y$ be in the relative interior of $V_{2}^{\prime}$, i.e. $\left(V_{2}^{\prime}\right)^{*}=\left\langle\pi^{\prime}\left(\xi_{0}\right), \ldots, \pi^{\prime}\left(\xi_{s}\right)\right\rangle$, where there exist real numbers $R^{\prime}>R$ such that $\left\|y-\xi_{0}\right\|=\cdots=\left\|y-\xi_{s}\right\|=R$ and $\|y-\eta\|>R^{\prime}$ for all $\eta$ in $\Lambda$ different from $\xi_{0}, \ldots, \xi_{s}$. We may assume the face $D_{1}^{\prime}$ to be of the form $\left\langle\pi^{\prime}\left(\xi_{0}\right), \ldots, \pi^{\prime}\left(\xi_{r}\right)\right\rangle$ for $r \leqslant s$. Thus there exist $z$ in $E^{\prime}$ and a real number $c$ such that $\left(\xi_{i}, z\right) \geqslant c$ for $0 \leqslant i \leqslant s$ with the equality holding if and only if $0 \leqslant i \leqslant r$. Let $\varepsilon$ be a small enough positive real number and let $x=y-\varepsilon z$. Then for $0 \leqslant i \leqslant s$ we have

$$
\left\|x-\xi_{i}\right\|^{2} \geqslant R^{2}+2 \varepsilon\{c-(z, y)\}+\varepsilon^{2}\|z\|^{2}
$$

with the equality holding if and only if $0 \leqslant i \leqslant r$. Moreover for all $\eta$ in $\Lambda$ different from $\xi_{0}, \ldots, \xi_{s}$, we have

$$
\|x-\eta\|=\|y-\eta-\varepsilon z\| \geqslant\|y-\eta\|-\varepsilon\|z\|>R^{\prime}-\varepsilon\|z\| .
$$

If $\varepsilon$ is small enough we obviously have

$$
\left(R^{\prime}-\varepsilon\|z\|\right)^{2}>R^{2}+2 \varepsilon\{c-(z, y)\}+\varepsilon^{2}\|z\|^{2},
$$

hence $D_{1}^{\prime}=\pi^{\prime}(D(x))$ and $x$ is in

$$
\begin{aligned}
\operatorname{rel} . i n t\left(V_{1}^{\prime}\right)=\left\{x \in E_{\psi}^{\prime} \mid\left\|x-\xi_{0}\right\|=\cdots=\right. & \left\|x-\xi_{r}\right\|<\|x-\eta\| \\
& \left.\quad \text { for all } \eta \text { in } \Lambda \neq \xi_{0}, \ldots, \xi_{r}\right\} .
\end{aligned}
$$

Thus we have (iii) as well as the fact that $\operatorname{Del}_{\psi}\left(E^{\prime} ; E, \Lambda\right)$ contains the faces of each of its members.

(4) We show next that if $D_{1}^{\prime}$ and $D_{2}^{\prime}$ are in $\operatorname{Del}_{\psi}\left(E^{\prime} ; E, \Lambda\right)$, then $D_{1}^{\prime} \cap D_{2}^{\prime}$ is a face of $D_{1}^{\prime}$ as well as that of $D_{2}^{\prime}$. Let $x$ and $y$ be in the respective relative interior of $\left(D_{1}^{\prime}\right)^{*}$ and $\left(D_{2}^{\prime}\right)^{*}$, hence there exist $\xi_{0}, \ldots, \xi_{r}$ and $\eta_{0}, \ldots, \eta_{s}$ in $\Lambda$ and positive real numbers $R_{1}$ and $R_{2}$ such that $\|x-\zeta\| \geqslant R_{1}$ for all $\zeta$ in $\Lambda$ with the equality holding if and only if $\zeta=\xi_{0}, \ldots, \xi_{r}$, and that $\|y-\zeta\|>$ $R_{2}$ for all $\zeta$ in $\Lambda$ with the equality holding if and only if $\zeta=\eta_{0}, \ldots, \eta_{s}$. Consider the "radial hyperplane" of the two spheres, one of radius $R_{1}$ with center at $x$ and the other of radius $R_{2}$ with center at $y$

$$
H=\left\{z \in E ; 2(y-x, z)=R_{1}^{2}-R_{2}^{2}+\|y\|^{2}-\|x\|^{2}\right\} .
$$

For each $j$ we have $R_{1}^{2} \leqslant\left\|x-\eta_{j}\right\|^{2}=R_{2}^{2}+2\left(y-x, \eta_{j}\right)+\|x\|^{2}-\|y\|^{2}$ with the equality holding if and only if $\eta_{j}=\xi_{i}$ for some $i$, i.e. $D_{2}^{\prime}=$ $\left\langle\pi^{\prime}\left(\eta_{0}\right), \ldots, \pi^{\prime}\left(\eta_{s}\right)\right\rangle$ is on the nonnegative side of $H$. Similarly for each $i$ we have

$$
R_{2}^{2} \leqslant\left\|y-\xi_{i}\right\|^{2}=R_{1}^{2}-2\left(y-x, \xi_{i}\right)+\|y\|^{2}-\|x\|^{2}
$$

with the equality holding if and only if $\xi_{i}=\eta_{j}$ for some $j$, i.e. $D_{1}^{\prime}=$ $\left\langle\pi^{\prime}\left(\xi_{0}\right), \ldots, \pi^{\prime}\left(\xi_{r}\right)\right\rangle$ is on the nonpositive side of $H$.

(5) It remains to show that the union of all $D^{\prime}$ in $\operatorname{Del}_{\psi}\left(E^{\prime} ; E, \Lambda\right)$ coincides 
with $E^{\prime}$. Obviously it is enough to show that $E^{\prime}$ coincides with the union $S$ of $\left(v^{\prime}\right)^{*}$ with $v^{\prime}$ running over $\operatorname{Sk}^{0}\left(\operatorname{Vor}\left(E_{\psi}^{\prime} ; E, \Lambda\right)\right)$. But $S$ is a closed subset of $E^{\prime}$, since $\left(v^{\prime}\right)^{*}$ is closed and $\left\{\left(v^{\prime}\right)^{*} ; v^{\prime} \in \operatorname{Sk}^{0}\left(\operatorname{Vor}\left(E_{\psi}^{\prime} ; E, \Lambda\right)\right)\right\}$ is a locally finite family in view of the fact that $\pi^{\prime}(\Lambda)$ is a lattice in $E^{\prime}$. Suppose there exists $x$ in $E^{\prime}$ and not in $S$. There exists then a small ball $\Sigma$ with center at $x$ contained in the open set $E^{\prime}-S$. Let us fix $u_{1}^{\prime}$ in $\operatorname{Sk}^{0}\left(\operatorname{Vor}\left(E_{\psi}^{\prime} ; E, \Lambda\right)\right.$ ) and a point $a$ in the interior of $\left(u_{1}^{\prime}\right)^{*}$. Then there exists $y$ in the interior of $\Sigma$ such that the line segment $\langle a, y\rangle$ meets none of $D^{\prime}$ in $\operatorname{Del}_{\psi}\left(E^{\prime} ; E, \Lambda\right)$ of codimension 2 , since the dimension of the join $\langle a, \Sigma\rangle$ is equal to that of $E^{\prime}$. There exist then a point $b$ on $\langle a, y\rangle$ and $u_{2}^{\prime}$ in $\operatorname{Sk}^{0}\left(\operatorname{Vor}\left(E_{\psi}^{\prime} ; E, \Lambda\right)\right)$ such that $b$ is one of the end points of the interval $\left(u_{2}^{\prime}\right)^{*} \cap\langle a, y\rangle$ and that the relative interior of $\langle b, y\rangle$ does not meet $S$. Thus $b$ is in the boundary of $S$, hence is in the relative interior of a codimension one face $\left(u_{2}^{\prime}\right)^{*} \cap\left(u_{3}^{\prime}\right)^{*}$ of $\left(u_{2}^{\prime}\right)^{*}$ by our choice of $y$. But the relative interior of $\left(u_{2}^{\prime}\right)^{*} \cap\left(u_{3}^{\prime}\right)^{*}$ is obviously in the interior of the union $\left(u_{2}^{\prime}\right)^{*} \cup\left(u_{3}^{\prime}\right)^{*}$, hence is in $S$, a contradiction.

REMARK. In the case of the ordinary Delony decomposition $E^{\prime}=E$, we have

$$
\operatorname{Sk}^{0}(\operatorname{Del}(E, \Lambda))=\Lambda
$$

since $V(\xi)^{*}=\xi$. In the general case, we need not have

$$
\operatorname{Sk}^{0}\left(\operatorname{Del}_{\psi}\left(E^{\prime} ; E, \Lambda\right)\right)=\pi^{\prime}(\Lambda) \text {. }
$$

Consider, for instance, the case where $E=\mathbf{R}^{2}$ with the usual bilinear form $\left(e_{i}, e_{j}\right)=\delta_{i j}$ and $e_{1}=(1,0), e_{2}=(0,1)$. Let $E^{\prime}$ be the line generated by $-e_{1}+2 e_{2}$, and $\psi=0$. We show below, however, that when $E, \Lambda$ and $E^{\prime}$ come from a graph, the equality holds if $\psi$ is chosen to be nondegenerate (cf. Proposition 7.6).

2. Stable and semistable Delony polyhedra. Let $E, E^{\prime}, E^{\prime \prime}$ and $\Lambda$ be as in the previous section.

The following notions of $\psi$-semistability and $\psi$-stability of Delony polyhedra were motivated by algebro-geometric considerations we make in the next chapter. They correspond to semistability and stability in the geometric invariant theory introduced by Mumford.

Defintion. For $\psi$ in $E^{\prime \prime}$ we call a Delony polyhedron $D$ in $\operatorname{Del}(E, \Lambda)$ $\psi$-semistable, if $D^{*} \cap E_{\psi}^{\prime} \neq \varnothing$, i.e. $\pi^{\prime \prime}\left(D^{\prime}\right) \ni \psi$. We denote by $K_{\psi}=K_{\psi}\left(E^{\prime}\right.$; $E, \Lambda)$ the set of $\psi$-semistable Delony polyhedra in $\operatorname{Del}(E, \Lambda)$. We denote by $K_{\psi}^{0}=K_{\psi}^{0}\left(E^{\prime} ; E, \Lambda\right)$ the subset of $K_{\psi}$ consisting of those $D$ for which rel.int $\left(D^{*}\right) \cap E_{\psi}^{\prime} \neq \varnothing$, i.e. $\pi^{\prime \prime}\left(\operatorname{rel} \operatorname{int}\left(D^{*}\right)\right) \ni \psi$.

By the definition of the Namikawa decomposition $\operatorname{Del}_{\psi}\left(E^{\prime} ; E, \Lambda\right)$, the map $D \mapsto \pi^{\prime}(D)$ is a bijection

$$
\pi^{\prime}: K_{\psi}^{0}\left(E^{\prime} ; E, \Lambda\right) \stackrel{\sim}{\rightarrow} \operatorname{Del}_{\psi}\left(E^{\prime} ; E, \Lambda\right) .
$$

Obviously $K_{\psi}$ consists of polyhedra in $K_{\psi}^{0}$ and their faces. 
We now claim there exists a map

$$
\mu: K_{\psi} \rightarrow K_{\psi}^{0}
$$

which is quasi-inverse to the inclusion $K_{\psi}^{0} \subset K_{\psi}$. Indeed, if $D$ is in $K_{\psi}$, we have $D^{*} \cap E_{\psi}^{\prime} \neq \varnothing$. There exists the smallest element $\mu(D)$ of $K_{\psi}^{0}$ such that $D<\mu(D)$, since $K_{\psi}$ consists of faces of elements of $K_{\psi}^{0}$ and since for $D_{1}$ and $D_{2}$ in $K_{\psi}^{0}$ with $D_{1} \cap D_{2} \neq \varnothing$ we have $D_{1} \cap D_{2} \in K_{\psi}^{0}$ in view of the corresponding property of the Namikawa decomposition $\operatorname{Del}_{\psi}\left(E^{\prime} ; E, \Lambda\right) . \mu(D)^{*}$ is the unique face $V$ of $D^{*}$ such that $\operatorname{rel} \operatorname{int}(V) \cap E_{\psi}^{\prime} \neq \varnothing$ and that $V \cap E_{\psi}^{\prime}=$ $D^{*} \cap E_{\psi}^{\prime}$.

Defintion. $D_{1}$ and $D_{2}$ in $K_{\psi}$ are said to be $\psi$-equivalent if $\mu\left(D_{1}\right)=\mu\left(D_{2}\right)$, i.e. $D_{1}^{*} \cap E_{\psi}^{\prime}=D_{2}^{*} \cap E_{\psi}^{\prime}$.

Each $\psi$-equivalence class has a unique element belonging to $K_{\psi}^{0}$, namely $\mu(D)$ for $D$ in the class. We have

$$
K_{\psi}\left(E^{\prime} ; E, \Lambda\right) / \psi \text {-equiv. } \stackrel{\mu}{\rightarrow} K_{\psi}^{0}\left(E^{\prime} ; E, \Lambda\right) \stackrel{\pi^{\prime}}{\rightarrow} \operatorname{Del}_{\psi}\left(E^{\prime} ; E, \Lambda\right) .
$$

Definition. A Delony polyhedron $D$ in $K_{\psi}\left(E^{\prime} ; E, \Lambda\right)$ is called $\psi$-stable if $D$ is the unique element $\psi$-equivalent to it. We denote by

$$
K_{\psi \text {-stable }}=K_{\psi \text {-stable }}\left(E^{\prime} ; E, \Lambda\right)
$$

the set of $\psi$-stable Delony polyhedra. Obviously $K_{\psi \text {-stable }} \subset K_{\psi}^{0}$.

Proposition 2.1. For a Delony polyhedron $D$ in $K_{\psi}\left(E^{\prime} ; E, \Lambda\right)$ the following are equivalent:

(1) $D$ is $\psi$-stable.

(2) $D$ is in $K_{\psi}^{0}$ and $\pi^{\prime}$ induces a bijection

$$
\pi^{\prime}: D \stackrel{\sim}{\rightarrow} \pi^{\prime}(D) \text {. }
$$

(3) $D$ is in $K_{\psi}^{0}$ and $\operatorname{dim} D=\operatorname{dim} \pi^{\prime}(D)$.

(4) $D$ is in $K_{\psi}^{0}$ and $\operatorname{dim} \pi^{\prime \prime}\left(D^{*}\right)=\operatorname{dim} E^{\prime \prime}$.

Proof. (1) $\Rightarrow(2)$. Suppose $D$ is $\psi$-stable, hence in particular $D$ is in $K_{\psi}^{0}$. If $\pi^{\prime}: D \rightarrow \pi^{\prime}(D)$ is not injective, there certainly exists an element of the boundary of $D$ whose image by $\pi^{\prime}$ is in the relative interior of $\pi^{\prime}(D)$. Let $D_{1}$ be the proper face of $D$ containing that point in its relative interior. Thus we have $\pi^{\prime}\left(D_{1}\right) \cap \operatorname{rel}$.int $\left(\pi^{\prime}(D)\right) \neq \varnothing$. In particular, $D_{1}$ is in $K_{\psi}$ but not in $K_{\psi}^{0}$. Moreover since $D_{1}<\mu\left(D_{1}\right)<D$, we have $\pi^{\prime}\left(D_{1}\right) \subset \pi^{\prime}\left(\mu\left(D_{1}\right)\right)<\pi^{\prime}(D)$. Thus we have $\mu\left(D_{1}\right)=D$, contradicting (1).

(2) is obviously equivalent to (3).

(3) is equivalent to (4). Indeed, we have $\operatorname{dim} E-\operatorname{dim} D^{*}=\operatorname{dim} D$ and $\operatorname{dim} E^{\prime}-\operatorname{dim} D^{*} \cap E_{\psi}^{\prime}=\operatorname{dim} \pi^{\prime}(D)$ by Proposition 1.6. Thus (3) is equivalent to $\operatorname{dim} E^{\prime \prime}=\operatorname{dim} D^{*}-\operatorname{dim} D^{*} \cap E_{\psi}^{\prime}$, which is equivalent to (4), since $\operatorname{dim} \pi^{\prime \prime}\left(D^{*}\right)=\operatorname{dim} D^{*}-\operatorname{dim}\left(\operatorname{rel} . i n t\left(D^{*}\right) \cap E_{\psi}^{\prime}\right)$ and $D$ is in $K_{\psi}^{0}$. 
Finally (4) implies (1). Indeed, suppose $D$ is in $K_{\psi}^{0}$ and is not $\psi$-stable. Then there exists a proper face $D_{1}$ of $D$ such that $D_{1}^{*} \cap E_{\psi}^{\prime}=D^{*} \cap E_{\psi}^{\prime}$. Since rel.int $\left(D^{*}\right) \cap E_{\psi}^{\prime} \neq \varnothing$ by assumption, we have

$$
\begin{aligned}
\operatorname{dim} \pi^{\prime \prime}\left(D^{*}\right) & =\operatorname{dim} D^{*}-\operatorname{dim} D^{*} \cap E_{\psi}^{\prime}=\operatorname{dim} D^{*}-\operatorname{dim} D_{1}^{*} \cap E^{\prime} \\
& \leqslant \operatorname{dim} D^{*}-\left(\operatorname{dim} D_{1}^{*}+\operatorname{dim} E_{\psi}^{\prime}-\operatorname{dim} E\right) \\
& =\operatorname{dim} E^{\prime \prime}-\left(\operatorname{dim} D_{1}^{*}-\operatorname{dim} D^{*}\right)<\operatorname{dim} E^{\prime \prime},
\end{aligned}
$$

a contradiction.

Corollary 2.2. If $D$ is $\psi$-stable and $D^{\prime}$ is a face of $D$, then $D^{\prime}$ is $\psi$-stable.

Proof. Obvious from (2).

We now compare Namikawa decompositions for different $\psi$ 's. For $\psi$ in $E^{\prime \prime}$, consider the intersection $P(\psi)=\cap \pi^{\prime \prime}\left(D^{*}\right)$ where $D$ runs over all the Delony polyhedra in $K_{\psi}=K_{\psi}\left(E^{\prime} ; E, \Lambda\right)$, i.e. $\pi^{\prime \prime}\left(D^{*}\right) \ni \psi$. Since $K_{\psi}$ is invariant under the translation action of $\Lambda \cap E^{\prime}$ and is finite modulo that action, we see that $P(\psi)$ is obtained as the intersection of only a finite number of $\pi^{\prime \prime}\left(D^{*}\right)$. Hence $P(\psi)$ is a bounded convex polyhedron of $E^{\prime \prime}$. Moreover, it is obvious that

$$
P(\psi)=\bigcap \pi^{\prime \prime}\left(D^{*}\right)
$$

and

$$
\operatorname{rel.int}(P(\psi))=\bigcap \pi^{\prime \prime}\left(\operatorname{rel} . \operatorname{int}\left(D^{*}\right)\right)
$$

where $D$ runs over all the Delony polyhedra in $K_{\psi}^{0}=K_{\psi}^{0}\left(E^{\prime} ; E, \Lambda\right)$, i.e. $\pi^{\prime \prime}\left(\operatorname{rel} . i n t\left(D^{*}\right)\right) \ni \psi$.

Defintion. We denote by $\operatorname{Par}\left(E^{\prime \prime}\right)$ the set of polyhedra $P$ in $E^{\prime \prime}$ of the form $P=P(\psi)$ for $\psi$ in $E^{\prime \prime}$.

Proposition 2.3. $\operatorname{Par}\left(E^{\prime \prime}\right)$ is a polyhedral decomposition of $E^{\prime \prime}$ by bounded convex polyhedra invariant under the translation action of $\pi^{\prime \prime}(\Lambda)$ with $\operatorname{Par}\left(E^{\prime \prime}\right) / \pi^{\prime \prime}(\Lambda)$ finite. Moreover, we have the following:

(i) For $P$ in $\operatorname{Par}\left(E^{\prime \prime}\right)$, the sets $K_{\psi}\left(E^{\prime} ; E, \Lambda\right), K_{\psi}^{0}\left(E^{\prime} ; E, \Lambda\right)$ and $K_{\psi \text {-stable }}\left(E^{\prime}\right.$; $E, \Lambda)$ of Delony polyhedra stays the same as long as $\psi$ is in $\operatorname{rel}$ int $(P)$.

(ii) For $P$ in $\operatorname{Par}\left(E^{\prime \prime}\right)$, we get the same Namikawa decomposition $\operatorname{Del}_{\psi}\left(E^{\prime}\right.$; $E, \Lambda)$ as long as $\psi$ is in $\operatorname{relint}(P)+\left(\Lambda \cap E^{\prime \prime}\right)$.

(iii) Let $P$ be in $\operatorname{Par}\left(E^{\prime \prime}\right)$ and let $\xi$ be in $\Lambda$. Then for $\psi$ in $\operatorname{rel}$.int $(P)$ and $\bar{\psi}$ in rel.int $(P)+\pi^{\prime \prime}(\xi)$, the translation by $\xi$ induces an isomorphism

$$
K_{\psi}^{0}\left(E^{\prime} ; E, \Lambda\right) \stackrel{\sim}{\rightarrow} K_{\bar{\psi}}^{0}\left(E^{\prime} ; E, \Lambda\right),
$$

hence the translation by, $\pi^{\prime}(\xi)$ induces an isomorphism

$$
\operatorname{Del}_{\psi}\left(E^{\prime} ; E, \Lambda\right) \stackrel{\sim}{\rightarrow} \operatorname{Del}_{\bar{\psi}}\left(E^{\prime} ; E, \Lambda\right) .
$$

(iv) Let $\bar{P}$ be a face of $P$ in $\operatorname{Par}\left(E^{\prime \prime}\right)$. For $\psi$ in $\operatorname{rel}$.int $(P)$ and $\bar{\psi}$ in $\operatorname{rel}$ int $(\bar{P})$, $\operatorname{Del}_{\psi}\left(E^{\prime} ; E, \Lambda\right)$ is a subdivision of $\operatorname{Del}_{\psi}\left(E^{\prime} ; E, \Lambda\right)$. 
Proof. The fact that $\operatorname{Par}\left(E^{\prime}\right)$ is a polyhedral decomposition is obvious as well as the invariance under $\pi^{\prime \prime}(\Lambda)$. (i), (ii) and (iii) are also obvious. (iv) follows from the following observation: for $D$ in $K_{\psi}^{0}$, we have $\pi^{\prime \prime}\left(D^{*}\right) \supset P \supset$ $\bar{P}$ hence there exists a face $\bar{D}^{*}$ of $D^{*}$ such that $\pi^{\prime \prime}\left(\operatorname{rel} \operatorname{int}\left(\bar{D}^{*}\right)\right) \ni \bar{\psi}$. Then we have $\pi^{\prime}(D) \subset \pi^{\prime}(\bar{D})$ with $\bar{D}$ in $K_{\bar{\psi}}^{0}$.

COROLlaRy 2.4. For $E, E^{\prime}, E^{\prime \prime}$ and $\Lambda$ fixed, there are only a finite number of different Namikawa decompositions of $E^{\prime}$.

3. Arrangement of hyperplanes. In this section, we introduce another kind of polyhedral decomposition, obtained by the arrangement of hyperplanes. The main reason for introducing it is that the polyhedral decomposition $\operatorname{Par}\left(E^{\prime \prime}\right)$ introduced at the end of the previous section is obtained by the arrangement of hyperplanes when $E$ comes from a graph (cf. \$7). We can also show that the Delony decomposition can sometimes be obtained by the arrangement of hyperplanes.

Let $F$ be a finite-dimensional real vector space and let $f_{1}, \ldots, f_{a}$ be linear forms on $F$, i.e. elements of the dual space $\check{F}=\operatorname{Hom}_{\mathbf{R}}(F, \mathbf{R})$. Suppose, moreover, that they generate $\check{F}$ over $\mathbf{R}$. For $1 \leqslant \alpha \leqslant a$ and $y$ in $F$, we denote by $M\left(f_{\alpha}, y\right)$ and $m\left(f_{\alpha}, y\right)$, respectively, the smallest integer not less than $f_{\alpha}(y)$ and the largest integer not more than $f_{\alpha}(y)$. Thus $m\left(f_{\alpha}, y\right)=M\left(f_{\alpha}, y\right)$ or $m\left(f_{\alpha}, y\right)=M\left(f_{\alpha}, y\right)-1$ according as $f_{\alpha}(y)$ is an integer or not. For $y$ in $F$, we denote by $A(y)$ the polyhedron in $F$ defined by

$$
A(y)=\left\{z \in F ; m\left(f_{\alpha}, y\right) \leqslant f_{\alpha}(z) \leqslant M\left(f_{\alpha}, y\right) \text { for all } \alpha\right\} .
$$

Note that if the set $\left\{f_{1}, \ldots, f_{a}\right\}$ is invariant under the multiplication by -1 , then we have

$$
A(y)=\left\{z \in F ; f_{\alpha}(z) \leqslant M\left(f_{\alpha}, y\right) \text { for all } \alpha\right\} .
$$

Proposition 3.1. The set $\operatorname{Arr}\left(F,\left\{f_{1}, \ldots, f_{a}\right\}\right)$ of polyhedra $A$ in $F$ of the form $A=A(y)$ for some $y$ in $F$ is a polyhedral decomposition of $F$ by bounded convex polyhedra, called the arrangement of hyperplanes. Moreover, it is invariant under the translation by elements of $\left\{g \in F ; f_{\alpha}(g)\right.$ integers for all $\left.\alpha\right\}$, which is contained in the set $\operatorname{Sk}^{0}\left(\operatorname{Arr}\left(F,\left\{f_{1}, \ldots, f_{a}\right\}\right)\right)$ of 0 -dimensional polyhedra.

Proof. The map $f$ sending $y$ to $f(y)=\left(f_{1}(y), \ldots, f_{a}(y)\right)$ is an embedding of $F$ into $\mathbf{R}^{a}$. Let $\left\{\varepsilon_{1}, \ldots, \varepsilon_{a}\right\}$ be the standard basis of $\mathbf{R}^{a}$ given by $\varepsilon_{\alpha}=$ $\left(\varepsilon_{\alpha 1}, \ldots, \varepsilon_{\alpha a}\right)$ with $\varepsilon_{\alpha \beta}=\delta_{\alpha \beta}$. Let $\left\{\check{\varepsilon}_{1}, \ldots, \check{\varepsilon}_{a}\right\}$ be the dual basis of the dual space of $\mathbf{R}^{a}$. Then we see by definition that

$$
\operatorname{Arr}\left(\mathbf{R}^{a},\left\{\check{\varepsilon}_{1}, \ldots, \check{\varepsilon}_{a}\right\}\right)
$$

coincides with the Delony decomposition $\operatorname{Del}\left(\mathbf{R}^{a} ; \mathbf{Z}^{a}\right)$ of $\mathbf{R}^{a}$ with respect to the lattice $\mathbf{Z}^{a}$ and the standard metric 


$$
\|x\|^{2}=\sum_{\alpha=1}^{a} \check{\varepsilon}_{\alpha}(x)^{2}
$$

on $\mathbf{R}^{a}$. Obviously $\operatorname{Arr}\left(F,\left\{f_{1}, \ldots, f_{a}\right\}\right)$ is the decomposition of $F$ induced by $f$ from $\operatorname{Del}\left(\mathbf{R}^{a} ; \mathbf{Z}^{a}\right)$, i.e. it consists of $A=f^{-1}(D)$ for $D$ in $\operatorname{Del}\left(\mathbf{R}^{a} ; \mathbf{Z}^{a}\right)$ with $f^{-1}($ rel.int $(D))$ nonempty. The rest of the proposition is immediate.

COROllary 3.2. Let $F$ and $G$ be a real vector space and a lattice in it, respectively. Let $\left\{f_{1}, \ldots, f_{a}\right\}$ be a subset of $\check{G}=\operatorname{Hom}_{\mathbf{Z}}(G, \mathbf{Z}) \subset \check{F}$ which generates $\dot{G}$. Then $\operatorname{Arr}\left(F,\left\{f_{1}, \ldots, f_{a}\right\}\right)$ is a polyhedral decomposition of $F$ invariant under the translation by elements of $G$. Moreover, the set $\operatorname{Sk}^{0}\left(\operatorname{Arr}\left(F,\left\{f_{1}, \ldots, f_{a}\right\}\right)\right)$ of 0 -dimensional polyhedra contains $G$ and it coincides with $G$ if and only if $\left\{f_{1}, \ldots, f_{a}\right\}$ is totally unimodular in the sense that $a$ subset of $\left\{f_{1}, \ldots, f_{a}\right\}$ is a Z-basis of $\check{G}$ if and only if it is an R-basis of $\check{F}$. Under the total unimodularity assumption, $\operatorname{Arr}\left(F,\left\{f_{1}, \ldots, f_{a}\right\}\right)$ coincides with the Delony decomposition $\operatorname{Del}(F ; G)$ of $F$ with respect to the metric on $F$ defined by

$$
\|y\|^{2}=\sum_{\alpha=1}^{a} f_{\alpha}(y)^{2} .
$$

Proof. Consider, as before, the embedding $f: F \rightarrow \mathbf{R}^{a}$ defined by $f(y)=$ $\left(f_{1}(y), \ldots, f_{a}(y)\right)$. The metric on $F$ is obviously the one induced from the standard metric on $\mathbf{R}^{a}$. Since $\left\{f_{1}, \ldots, f_{a}\right\}$ generates $\check{G}$, we have $G=f^{-1}\left(\mathbf{Z}^{a}\right)$. Let $D$ be in $\operatorname{Del}\left(\mathbf{R}^{a} ; \mathbf{Z}^{a}\right)$ with $f^{-1}(\operatorname{rel}$.int $(D))$ nonempty, and let $A=f^{-1}(D)$. Under the total unimodularity assumption, we see immediately that $A$ is 0 -dimensional if and only if $A=\{g\}$ for $g$ in $G$, hence $D=\{f(g)\}$. Let $D^{\prime}$ be in $\operatorname{Del}(F ; G)$. Then by definition there exists $y$ in $F$ such that $D^{\prime}$ is the convex hull of those $g$ in $G$ with $\|y-g\|$ minimal. Consider $D=D(f(y))$ in $\operatorname{Del}\left(\mathbf{R}^{a} ; \mathbf{Z}^{a}\right)$. It is the convex hull of those $\xi$ in $\mathbf{Z}^{a}$ with $\|f(y)-\xi\|$ minimal. Thus $D^{\prime}$ is obviously contained in $f^{-1}(D)$, and the convex polyhedron $f^{-1}(D)$ is the convex hull of its 0-dimensional faces, which are necessarily in $G$, as we saw above. Thus we have $D^{\prime}=f^{-1}(D)$.

REMARK. The Delony decompositions in dimensions less than four are necessarily of this form. See Voronoi [34].

PROBLEM. What are the normal forms of maximal totally unimodular sets in higher dimension? See Dickson [6], Hoffman-Kruskal [12] and HellerTompkins [13].

4. Graphs. In this paper we deal with finite graphs in the most general sense, i.e. we allow loops and multiple edges. For details we refer the reader to standard textbooks in graph theory, for example Berge [2].

Definition. A (finite) graph $\Gamma=\{I, J\}$ consists of a finite set $I$ of vertices and a finite set $J$ of edges together with the incidence relation which assigns 
two vertices, called end points, to each edge. When two end points of an edge coincide, we call the edge a loop. $\Gamma$ is called connected if starting from a vertex we can reach any other vertex by following a path.

Given a graph $\Gamma$, we assign and fix an arbitrary orientation. i.e. we fix maps $J \rightrightarrows I$ assigning to each edge $j$ its initial and terminal vertices. Our results are independent of the particular orientation we choose.

The motivation for our discussion of graphs is the following example, which we exclusively deal with in subsequent chapters.

EXAMPLE. Let $X$ be a reduced complete algebraic curve with at most ordinary double points over an algebraically closed field. Then $X$ decomposes into irreducible components $X=\cup_{i \in I} X_{i}$. Let $\left\{Q_{j}\right\}_{j \in J}$ be the set of double points of $X$. Then $Q_{j}$ is either (1) an ordinary double point of an irreducible component $X_{i}$ or (2) a transversal intersection of irreducible components $X_{i}$ and $X_{i^{\prime}}$. In this case we associate to $X$ a connected graph $\Gamma(X)=\{I, J\}$ where (1) $j$ in $J$ is a loop at $i$ if $Q_{j}$ is a double point of $X_{i}$, while (2) $j$ in $J$ is an edge joining $i$ and $i^{\prime}$ if $Q_{j}$ is a transversal intersection of $X_{i}$ and $X_{i^{\prime}}$. Note that any graph in our sense appears as the graph of an algebraic curve $X$ as in this example. $\Gamma(X)$ is connected if and only if $X$ is connected (see Figure 2).
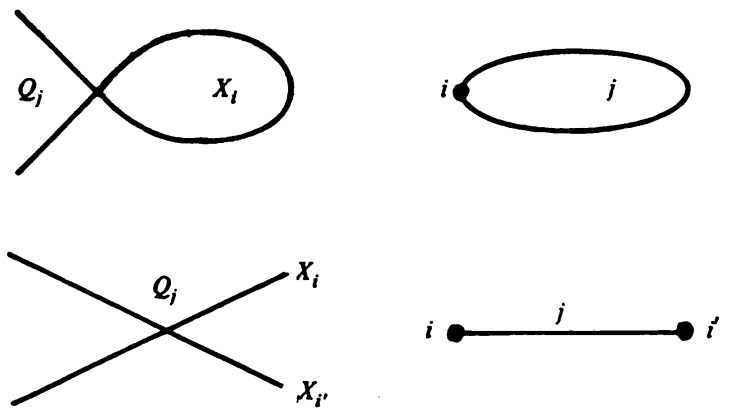

FIGURE 2

Once we fix an orientation, we can define a chain complex $C .(\Gamma, \mathbf{Z})$, where $C_{0}(\Gamma, \mathbf{Z})=\bigoplus_{i \in I} \mathbf{Z} v_{i}, \quad C_{1}(\Gamma, \mathbf{Z})=\bigoplus_{j \in J} \mathbf{Z} e_{j}$ with $\left\{v_{i}\right\}_{i \in I}$ and $\left\{e_{j}\right\}_{j \in J}$ canonically defined $\mathbf{Z}$-bases, and the boundary map

$$
\text { a: } C_{1}(\Gamma, \mathbf{Z}) \rightarrow C_{0}(\Gamma, \mathbf{Z})
$$

defined by

$$
\partial e_{j}= \begin{cases}0 & \text { if } j \text { is a loop, } \\ v_{i}-v_{i^{\prime}} & \text { if } j \text { is from } i \text { to } i^{\prime} .\end{cases}
$$

Since we have canonical bases, we have canonical pairings [, ] on $C_{0}(\Gamma, Z)$ and $($,$) on C_{1}(\Gamma, Z)$ defined by

$$
\left[v_{i}, v_{i^{\prime}}\right]=\delta_{i i^{\prime}}, \quad\left(e_{j}, e_{j^{\prime}}\right)=\delta_{i j^{\prime}}
$$


Thus we can always identify the cochain complex $C \cdot(\Gamma, \mathbf{Z})$ with the chain complex $C_{.}(\Gamma, \mathbf{Z})$ via these pairings. The coboundary map then becomes

$$
\delta: C_{0}(\Gamma, \mathbf{Z}) \rightarrow C_{1}(\Gamma, \mathbf{Z})
$$

defined by

$$
\delta v_{i}=\sum_{j \in J}\left[v_{i}, \partial e_{j}\right] e_{j} .
$$

Thus $\partial$ and $\delta$ are adjoint to each other, i.e. we have $(\delta x, y)=[x, \partial y]$ for $x$ in $C_{0}(\Gamma, \mathbf{Z})$ and $y$ in $C_{1}(\Gamma, \mathbf{Z})$.

Definition. For a subset $I^{\prime}$ of $I$, we denote by $v\left(I^{\prime}\right)$ the element of $C_{0}(\Gamma, \mathbf{Z})$ defined by

$$
v\left(I^{\prime}\right)=\sum_{i \in I^{\prime}} v_{i}
$$

For a subset $J^{\prime}$ of $J$, we denote by $e\left(J^{\prime}\right)$ the element of $C_{1}(\Gamma, \mathbf{Z})$ defined by

$$
e\left(J^{\prime}\right)=\sum_{j \in J^{\prime}} e_{j}
$$

Let $I_{1}, \ldots, I_{c}$ be the sets of vertices in the connected components of $\Gamma$. Then

$$
H^{0}(\Gamma, \mathbf{Z})=\underset{1<\alpha<c}{\bigoplus} \mathbf{Z} v\left(I_{\alpha}\right)
$$

$H_{0}(\Gamma, \mathbf{Z})$ is the dual free $\mathbf{Z}$-module, hence has rank equal to $c$, the number of connected components.

By the adjointness of $\partial$ and $\delta$, we get the following decompositions orthogonal with respect to the pairings:

$$
C_{0}(\Gamma, \mathbf{R})=H^{0}(\Gamma, \mathbf{R}) \oplus \partial C_{1}(\Gamma, \mathbf{R}), \quad C_{1}(\Gamma, \mathbf{R})=H_{1}(\Gamma, \mathbf{R}) \oplus \delta C_{0}(\Gamma, \mathbf{R}) .
$$

Moreover $\partial$ and $\delta$ induce isomorphisms

$$
\partial: \delta C_{0}(\Gamma, \mathbf{R}) \stackrel{\sim}{\rightarrow} \partial C_{1}(\Gamma, \mathbf{R}), \quad \delta: \partial C_{1}(\Gamma, \mathbf{R}) \stackrel{\sim}{\rightarrow} \delta C_{0}(\Gamma, \mathbf{R}),
$$

where $\mathbf{R}$ is the field of real numbers.

For a subset $J^{\prime}$ of $J$, we consider the spanning subgraph $\left\{I, J^{\prime}\right\}$ of $\Gamma=$ $\{I, J\}$, with $I$ as the set of vertices, $J^{\prime}$ as the set of edges and the orientation induced from $\Gamma$. We can consider the chain complex $C\left(\left\{I, J^{\prime}\right\}, Z\right)=$ $C \cdot\left(\left\{I, J^{\prime}\right\}, \mathbf{Z}\right)$, the boundary and coboundary maps $\partial_{J^{\prime}}, \delta_{J^{\prime}}$ for this graph. Obviously we have

$$
\begin{aligned}
& \partial_{J^{\prime}} e_{j}=\partial e_{j} \text { for } j \text { in } J^{\prime}, \text { and } \\
& \delta_{J^{\prime}, v_{i}}=\sum_{j^{\prime} \in J^{\prime}}\left[v_{i}, \partial e_{j^{\prime}}\right] e_{j^{\prime}} \text { for } i \text { in } I .
\end{aligned}
$$

The motivation for the introduction of such subgraphs is the following:

EXAMPLE. Let $X$ be an algebraic curve as in the previous example. For a subset $J^{\prime}$ of $J$, let $\sigma\left(J^{\prime}\right): X\left(J^{\prime}\right) \rightarrow X$ be the partial normalization of $X$ obtained 
by the blowing up along $\left\{Q_{j}\right\}_{j \in J-J^{\prime}}$. Then obviously the graph for $X\left(J^{\prime}\right)$ is the subgraph $\left\{I, J^{\prime}\right\}$.

Definition. The cyclomatic number $h(\Gamma)$ of a graph $\Gamma$ is defined as

$$
h(\Gamma)=\operatorname{rank} H_{1}(\Gamma, \mathbf{Z})=\operatorname{rank} H^{1}(\Gamma, \mathbf{Z}) .
$$

Proposition 4.1. For a graph $\Gamma=\{I, J\}$ with $c$ connected components, we have

$$
h(\Gamma)=|J|-|I|+c
$$

where $|J|$ and $|I|$ denote the cardinalities.

This is a standard result in graph theory and is a straightforward consequence of the Euler-Poincaré lemma.

We use the following Lemmas 4.3 and 4.4 and Corollary 4.5 later in Chapter II, $\$ 10$ and $\$ 11$.

Definimion. For a subset $J^{\prime}$ of $J$, we denote by $d\left(J^{\prime}\right)$ the element of $C_{0}(\Gamma, \mathbf{Z})$, the degree of the subgraph $\left\{I, J^{\prime}\right\}$, defined by

$$
d\left(J^{\prime}\right)=\sum_{i \in I} d\left(J^{\prime}\right)_{i} v_{i}
$$

where $d\left(J^{\prime}\right)_{i}$ is the number of edges, regardless of the orientation, in $J^{\prime}$ at least one of whose end points is at $i$, with loops at $i$ counted twice.

LEMMA 4.2. For a subset $J^{\prime}$ of $J$, we have

$$
\left[v(I), d\left(J^{\prime}\right)\right]=2\left|J^{\prime}\right| .
$$

This is again standard in graph theory, and can be easily proved, since on both sides of the equality, each edge is counted twice by definition.

LEMMA 4.3. For a subset $I^{\prime}$ of $I$ and a subset $J^{\prime}$ of $J$, the cardinality of the set of edges $j^{\prime}$, regardless of the orientation, in $J^{\prime}$ one of whose end points is in $I^{\prime}$ and the other is in $I-I^{\prime}$ is equal to

$$
\left(\delta_{J}, v\left(I^{\prime}\right), \delta_{J}, v\left(I^{\prime}\right)\right) \text {. }
$$

Especially $\left(\delta v\left(I^{\prime}\right), \delta v\left(I^{\prime}\right)\right)$ is the cardinality of the set of edges in $J$ joining $I^{\prime}$ and $I-I^{\prime}$, regardless of the orientation.

Proof. Since $\delta_{J}, v(I)=0$, we have

$$
\begin{aligned}
& \left(\delta_{J}, v\left(I^{\prime}\right), \delta_{J}, v\left(I^{\prime}\right)\right)=-\left(\delta_{J}, v\left(I-I^{\prime}\right), \delta_{J}, v\left(I^{\prime}\right)\right) \\
& =-\left(\delta_{J}, v\left(I-I^{\prime}\right), \sum_{j^{\prime} \in J^{\prime}}\left(\delta_{J^{\prime}} v\left(I^{\prime}\right), e_{j^{\prime}}\right) e_{j^{\prime}}\right) \\
& =-\sum_{j^{\prime} \in J^{\prime}} \sum_{i \in I^{\prime}, i^{\prime} \in I-I^{\prime}}\left[v_{i}, \partial e_{j^{\prime}}\right]\left[v_{i^{\prime}}, \partial e_{j^{\prime}}\right],
\end{aligned}
$$

which is equal to the cardinality of the set of $j^{\prime}$ in $J^{\prime}$ one of whose end points is in $I^{\prime}$ and the other end point is in $I-I^{\prime}$, since $\left[v_{i}, \partial e_{j}\right]\left[v_{i^{\prime}}, \partial e_{j^{\prime}}\right]=0$ or -1 . 
COROLlary 4.4. For a subset $I^{\prime}$ of $I$ and a subset $J^{\prime}$ of $J$, the cardinality of the set $J^{\prime}\left(I^{\prime}\right)$ of edges in $J^{\prime}$ both of whose end points are in $I^{\prime}$ is equal to

$$
\left[v\left(I^{\prime}\right), d\left(J^{\prime}\right) / 2\right]-\left(\delta_{J^{\prime}} v\left(I^{\prime}\right), \delta_{J^{\prime}}, v\left(I^{\prime}\right)\right) / 2
$$

Proof. Immediate from the two previous lemmas.

Definition. A tree is a connected graph without any cycle, i.e. the cyclomatic number zero. A spanning tree of a connected graph $\Gamma=\{I, J\}$ is a spanning subgraph $\left\{I, J^{\prime}\right\}$ which is a tree, i.e. a minimal connected spanning subgraph. A spanning forest of a not necessarily connected graph is a spanning subgraph which induces a tree for each connected component.

For details we refer the reader again to standard textbooks in graph theory, for example Berge [2]. One more fact which is relevant to us is the following which is again standard.

Proposition 4.5. Let $\Gamma=\{I, J\}$ be connected. Then for a spanning tree $\left\{I, J^{\prime}\right\}$ we have $\left|J^{\prime}\right|=|I|-1$, in particular $\left|J-J^{\prime}\right|=h(\Gamma)$.

Let $\rho: C_{1}(\Gamma, Z) \rightarrow H^{1}(\Gamma, Z)$ be the canonical surjective map, whose kernel is $\delta C_{0}(\Gamma, \mathbf{Z})$ by definition.

KIRCHHOFF-TRENT'S THEOREM. For a graph $\Gamma$, the number of spanning forests in $\Gamma$, called the complexity of $\Gamma$, is equal to the index

$$
\left[\partial C_{1}(\Gamma, \mathbf{Z}): \partial \delta C_{0}(\Gamma, \mathbf{Z})\right]=\left[H^{1}(\Gamma, \mathbf{Z}): \rho H_{1}(\Gamma, \mathbf{Z})\right],
$$

which is also equal to the absolute value of the discriminant of the pairing

$$
(,): H_{1}(\Gamma, \mathbf{Z}) \times H_{1}(\Gamma, \mathbf{Z}) \rightarrow \mathbf{Z}
$$

induced by $($,$) on C_{1}(\Gamma, Z)$, where we define the discriminant to be 1 when $H_{1}(\Gamma, \mathbf{Z})=0$.

For the proof of the fact that the complexity of $\Gamma$ is equal to the index $\left[\partial C_{1}(\Gamma, \mathbf{Z}): \partial \delta C_{0}(\Gamma, \mathbf{Z})\right]$, we refer the reader to Bryant [3, p. 115]. Although $\Gamma$ is assumed to be connected there, the modification required in the general case is immediate. The rest of the theorem follows easily from the canonical isomorphisms

$$
\begin{aligned}
\partial C_{1}(\Gamma, \mathbf{Z}) / \partial \delta C_{0}(\Gamma, \mathbf{Z}) & \approx C_{1}(\Gamma, \mathbf{Z}) /\left\{H_{1}(\Gamma, \mathbf{Z})+\delta C_{0}(\Gamma, \mathbf{Z})\right\} \\
& \stackrel{\sim}{\rightarrow} H^{1}(\Gamma, \mathbf{Z}) / \rho H_{1}(\Gamma, \mathbf{Z}) .
\end{aligned}
$$

Definition. An element $\gamma$ in $H_{1}(\Gamma, \mathbf{Z})$ is called a (graph-theoretical) cycle if $\left(\gamma, e_{j}\right)=0$ or \pm 1 for any $j$ in $J$. An element $\omega$ in $\delta C_{0}(T, Z)$ is called a (graph-theoretical) cocycle, if there exists a subset $I^{\prime}$ of $I$ such that $\omega=\delta v\left(I^{\prime}\right)$, hence necessarily $\left(\omega, e_{j}\right)=0$ or \pm 1 for any $j$ in $J$.

Let $\{I, T\}$ be a spanning forest of a graph $\Gamma=\{I, J\}$. Then as is well known in graph theory (cf. Berge [2, p. 26]), there exist Z-bases $\left\{\gamma_{T_{j}}\right.$; $j \in J-T\}$ and $\left\{\omega_{T, t} ; t \in T\right\}$ of $H_{1}(\Gamma, \mathbf{Z})$ and $\delta C_{0}(\Gamma, \mathbf{Z})$, respectively, de- 
fined as follows: for $j$ in $J-T$, the spanning subgraph $\{I, T \cup\{j\}\}$ has the cyclomatic number one. Thus there is a unique cycle $\gamma_{T_{j}}$ in this subgraph with $\left(\gamma_{T, j}, e_{j}\right)=1$. Similarly for $t$ in $T$, only one of the connected components of $\{I, T\}$ breaks up into two for the spanning subgraph $\{I, T-\{t\}\}$. Let $I_{t}$ be the set of vertices in the one of these two components in which $t$ has its initial vertex. Then we let $\omega_{T, t}=\delta v\left(I_{t}\right)$.

The following two lemmas are obvious from what we have seen so far. They will be useful in determining codimension one faces of Voronoi polyhedra in Proposition 5.2.

LEMMA 4.6. Let $\gamma$ be a cycle for a graph $\Gamma=\{I, J\}$ and let $J^{\prime}=\{j \in J$; $\left.\left(\gamma, e_{j}\right) \neq 0\right\}$ and $\Gamma^{\prime}=\left\{I, J^{\prime}\right\}$. Then the following are equivalent and in this case $\gamma$ is called an elementary cycle.

(i) $\gamma \neq 0$ and is minimal in the sense that it cannot be written nontrivially as a sum $\gamma=\gamma_{1}+\gamma_{2}$ of cycles with $\left\{j \in J ;\left(\gamma_{1}, e_{j}\right) \neq 0\right\}$ and $\left\{j \in J ;\left(\gamma_{2}, e_{j}\right) \neq\right.$ $0\}$ disjoint.

(ii) $H_{1}\left(\Gamma^{\prime}, \mathbf{R}\right)$ is one dimensional. In this case $\gamma$ generates this space.

(iii) $H^{1}\left(\Gamma^{\prime}, \mathbf{R}\right)$ is one dimensional.

(iv) $\left\{\rho\left(e_{j}\right) ; j \in J-J^{\prime}\right\}$ spans a codimension one subspace of $H^{1}(\Gamma, \mathbf{R})$, where $\rho: C_{1}(\Gamma, \mathbf{R}) \rightarrow H^{1}(\Gamma, \mathbf{R})$ is the canonical projection.

(v) There exist a spanning forest $\{I, T\}$ of $\Gamma$ and $j \in J-T$ such that $\gamma= \pm \gamma_{T, j}$

LEMMA 4.7. Let $\omega$ be a cocycle for a graph $\Gamma=\{I, J\}$ and let $J_{0}=\{j \in J$; $\left.\left(\omega, e_{j}\right)=0\right\}$ and $\Gamma_{0}=\left\{I, J_{0}\right\}$. Then the following are equivalent and in this case $\omega$ is called an elementary cocycle.

(i) $\omega \neq 0$ and is minimal in the sense that it cannot be written nontrivially as a sum $\omega=\omega_{1}+\omega_{2}$ of cocycles with $\left\{j \in J ;\left(\omega_{1}, e_{j}\right) \neq 0\right\}$ and $\left\{j \in J ;\left(\omega_{2}, e_{j}\right)\right.$ $\neq 0\}$ disjoint.

(ii) For the projection $P_{0}: C_{1}(\Gamma, \mathbf{R}) \rightarrow C_{1}\left(\Gamma_{0}, \mathbf{R}\right)$ defined by $P_{0}\left(e_{j}\right)=e_{j}$ or 0 according as $j \in J_{0}$ or not, the intersection $\operatorname{ker}\left(P_{0}\right) \cap \delta C_{0}(\Gamma, \mathbf{R})$ is one dimensional. In this case $\omega$ generates this space.

(iii) All but one of the connected components of $\Gamma$ remain connected for $\Gamma_{0}$ and the remaining component of $\Gamma$ breaks up into exactly two components of $\Gamma_{0}$. In this case $\omega=\delta v\left(I^{\prime}\right)$, where $I^{\prime}$ is the set of vertices in one of these two components of $\Gamma_{0}$.

(iv) $\left\{\partial e_{j} ; j \in J_{0}\right\}$ spans a codimension one subspace of $\partial C_{1}(\Gamma, \mathbf{R})$.

(v) There exist a spanning forest $\{I, T\}$ of $\Gamma$ and $t \in T$ such that $\omega=$ $\pm \omega_{T, \ell^{*}}$

5. Voronoi and Delony decompositions for a graph. Let $\Gamma=\{I, J\}$ be a graph which need not be connected. We apply our results in $\$ 2$ to the 
following:

$$
E=C_{1}(\Gamma, \mathbf{R})=\bigoplus_{j \in J} \mathbf{R} e_{j}
$$

with the canonical pairing $\left(e_{j}, e_{j^{\prime}}\right)=0$ or 1 , according as $j \neq j^{\prime}$ or $j=j^{\prime}$ and the lattice

$$
\Lambda=C_{1}(\Gamma, \mathbf{Z})=\bigoplus_{j \in J} \mathbf{Z} e_{j}
$$

We have seen in the previous section that if

$$
E^{\prime}=H_{1}(\Gamma, \mathbf{R}) \text { and } E^{\prime \prime}=\delta C_{0}(\Gamma, \mathbf{R})
$$

then we have an orthogonal decomposition $E=E^{\prime} \oplus E^{\prime \prime}$. Let $\pi^{\prime}: E \rightarrow E^{\prime}$ and $\pi^{\prime \prime}: E \rightarrow E^{\prime \prime}$ be the orthogonal projections as before. Then as we have seen, we have $\partial \circ \pi^{\prime \prime}=\partial$ and $\partial: E \rightarrow C_{0}(\Gamma, \mathbf{R})$ induces an isomorphism $E^{\prime} \stackrel{\sim}{\rightarrow} \partial E$. Similarly for the canonical surjection $\rho: E \rightarrow H^{1}(\Gamma, \mathbf{R})$, we have $\rho \circ \pi^{\prime}=\rho$ and $\rho$ induces an isomorphism $E^{\prime} \stackrel{\sim}{\rightarrow} H^{1}(\Gamma, \mathbf{R})$. Obviously $\Lambda \cap E^{\prime}$ $=H_{1}(\Gamma, \mathbf{Z})$ and $\Lambda \cap E^{\prime \prime}=\delta C_{0}(\Gamma, \mathbf{Z})$ are lattices in $E^{\prime}$ and $E^{\prime \prime}$, respectively. Moreover, $\pi^{\prime}(\Lambda)$ and $\pi^{\prime \prime}(\Lambda)$ are lattices in $E^{\prime}$ and $E^{\prime \prime}$, respectively, since $\pi^{\prime}(\Lambda)$ can be identified with $H^{1}(\Gamma, \mathbf{Z})$ by $\rho$, and $\pi^{\prime \prime}(\Lambda)$ with $\partial C_{1}(\Gamma, \mathbf{Z})$ by $\partial$. Hence our requirements in $\$ 2$ are all satisfied.

Definition. For a graph $\Gamma$, we denote $K(\Gamma)=\operatorname{Del}(E, \Lambda)$ and $\operatorname{Vor}(\Gamma)=$ $\operatorname{Vor}(E, \Lambda)$. We call polyhedra in them Delony and Voronoi polyhedra for $\Gamma$, respectively.

We have $\mathrm{Sk}^{0}(K(\Gamma))=\Lambda=C_{1}(\Gamma, \mathbf{Z})$ and $\mathrm{Sk}^{0}(\operatorname{Vor}(\Gamma))=\Lambda+e(J) / 2$.

DefinITION. For a subset $J^{\prime}$ of $J$, we denote

$$
V_{J^{\prime}}(0)=\left\{\sum_{j^{\prime} \in J^{\prime}} \lambda_{j^{\prime}} e_{j^{\prime}} ; \lambda_{j^{\prime}} \text { real with }\left|\lambda_{j^{\prime}}\right| \leqslant 1 / 2\right\} .
$$

$V_{J}(0)$ is easily seen to be the top-dimensional Voronoi polyhedron containing 0 , i.e.

$$
V_{J}(0)=\{x \in E ;\|x\| \leqslant\|x-\xi\| \text { for all } \xi \text { in } \Lambda\},
$$

since $E=\mathbf{R}^{|J|}$ is the standard Euclidean space and $\Lambda=\mathbf{Z}^{|J|}$. Similarly $V_{J},(0)$ is the top-dimensional Voronoi polyhedron containing 0 for the graph $\left\{I, J^{\prime}\right\}$.

Thus we easily see the following:

Proposition 5.1. A Delony polyhedron $D$ in $K(\Gamma)$ is of the form

$$
D=b+V_{J "}(0)
$$

where $J^{\prime \prime}$ is a subset of, $J$ and $b$ is an element of $E$ such that $b-e\left(J^{\prime \prime}\right) / 2$ is in $\Lambda$. We call $b=b(D)$ the barycenter of $D$ and $J^{\prime \prime}=\operatorname{Supp}(D)$ the support of $D$. The dual Voronoi polyhedron is

$$
D^{*}=b(D)+V_{J-J^{\prime \prime}}(0)
$$


Thus if $b(D)=\xi+e\left(J^{\prime \prime}\right) / 2$ for $\xi$ in $\Lambda$, then

$$
D=\left\{\xi+\sum_{j^{\prime \prime} \in J^{\prime \prime}} t_{j^{\prime \prime}} e_{j^{\prime \prime}} ; t_{j^{\prime \prime}} \text { real with } 0 \leqslant t_{j^{\prime \prime}}<1\right\} .
$$

We now come to the main result of this section comparing the Voronoi decomposition of $E$ with those of $E^{\prime}$ and $E^{\prime \prime}$ for a graph. This is crucial to our subsequent analysis of Namikawa decompositions for a graph. See Proposition 5.5 due to Mumford for a dual result concerning the Delony decompositions.

Proposition 5.2. For a graph $\Gamma=\{I, J\}$, we have the following:

(1) $\pi^{\prime}\left(V_{J}(0)\right)$ is the top-dimensional Voronoi polyhedron containing 0 in $E^{\prime}$ with respect to the restriction of $(,$,$) and the lattice \Lambda \cap E^{\prime}=H_{1}(\Gamma, \mathrm{Z})$, hence

$$
\operatorname{Vor}\left(E^{\prime}, \Lambda \cap E^{\prime}\right)=\left\{H_{1}(\Gamma, \mathbf{Z}) \text {-translates of faces of } \pi^{\prime}\left(V_{J}(0)\right)\right\} \text {. }
$$

Moreover, we have

$$
\pi^{\prime}\left(V_{J}(0)\right)=\left\{x \in E^{\prime} ;(x, \gamma) \leqslant(\gamma, \gamma) / 2 \text { for any elementary cycle } \gamma\right\} .
$$

These inequalities are irredundant in the sense that for each elementary cycle $\gamma$, the intersection of this with the hyperplane $H=\left\{x \in E^{\prime} ;(x, \gamma)=(\gamma, \gamma) / 2\right\}$ is its codimension one face.

(2) $\pi^{\prime \prime}\left(V_{J}(0)\right)$ is the top-dimensional Voronoi polyhedron containing 0 in $E^{\prime \prime}$ with respect to the restriction of $\left(\right.$, , ) and the lattice $\Lambda \cap E^{\prime \prime}=\delta C_{0}(\Gamma, \mathrm{Z})$, hence

$\operatorname{Vor}\left(E^{\prime \prime}, \Lambda \cap E^{\prime \prime}\right)=\left\{\delta C_{0}(\Gamma, \mathbf{Z})\right.$-translates of faces of $\left.\pi^{\prime \prime}\left(V_{J}(0)\right)\right\}$.

Moreover, we have

$$
\pi^{\prime \prime}\left(V_{J}(0)\right)=\left\{x \in E^{\prime \prime} ;(x, \omega) \leqslant(\omega, \omega) / 2 \text { for any elementary cocycle } \omega\right\} .
$$

These inequalities are irredundant in the sense that for each elementary cocycle $\omega$, the intersection of this with the hyperplane $H=\left\{x \in E^{\prime \prime} ;(x, \omega)=\right.$ $(\omega, \omega) / 2\}$ is its codimension one face.

Proof. $\pi^{\prime}\left(V_{J}(0)\right)$ is contained in the top-dimensional Voronoi polyhedron of $E^{\prime}$ containing 0 , which is of the form

$$
\left\{x \in E^{\prime} ;\|x\| \leqslant\|x-\xi\| \text { for all } \xi \text { in } \Lambda \cap E^{\prime}\right\} .
$$

Indeed for $\left|\lambda_{j}\right| \leqslant 1 / 2$ for $j$ in $J$ and $\xi$ in $\Lambda \cap E^{\prime}$, we have

$$
\begin{aligned}
\left(\pi^{\prime}\left(\sum \lambda_{j} e_{j}\right), \xi\right) & =\left(\sum \lambda_{j} e_{j}, \xi\right)=\sum \lambda_{j}\left(e_{j}, \xi\right) \\
& \leqslant \sum\left|\lambda_{j}\right|\left(e_{j}, \xi\right)^{2} \leqslant \sum\left(e_{j}, \xi\right)^{2} / 2=(\xi, \xi) / 2,
\end{aligned}
$$

since $\left(e_{j}, \xi\right)$ is an integer for all $j$ in $J$ by assumption.

Since $\pi^{\prime}\left(V_{J}(0)\right)$ is a convex polyhedron in $E^{\prime}$, it is enough, for the proof of (1), to show that the codimension one faces of $\pi^{\prime}\left(V_{J}(0)\right)$ are determined exactly by hyperplanes $H$ in $E^{\prime}$ of the form 


$$
H=\left\{x \in E^{\prime} ;(x, \gamma)=(\gamma, \gamma) / 2\right\}
$$

for an elementary cycle $\gamma$. By definition $V_{J}(0)$ is the convex hull of its vertices which are of the form $e\left(J^{\prime}\right) / 2-e\left(J-J^{\prime}\right) / 2$ for a subset $J^{\prime}$ of $J$. Thus $\pi^{\prime}\left(V_{J}(0)\right)$ is the convex hull of elements of the form

$$
\pi^{\prime}\left(e\left(J^{\prime}\right)-e\left(J-J^{\prime}\right)\right) / 2 \text {. }
$$

Let $a \neq 0$ be an element of $E^{\prime}$ such that for a real number $c$ the hyperplane

$$
H=\left\{x \in E^{\prime} ;(a, x)=c\right\}
$$

determines a codimension one face of $\pi^{\prime}\left(V_{J}(0)\right)$, i.e. $\left(a, \pi^{\prime}(y)\right)=(a, y) \leqslant c$ holds for all $y$ in $V_{J}(0)$ and $H \cap \pi^{\prime}\left(V_{J}(0)\right)$ is of codimension one in $E^{\prime}$. Since this intersection is determined by the vertices lying on it, there exist subsets $J_{1}^{\prime}, \ldots, J_{m}^{\prime}$ of $J$ such that $\left(a,\left(e\left(J_{\alpha}^{\prime}\right)-e\left(J-J_{\alpha}^{\prime}\right)\right) / 2\right)=c$ for $\alpha=1, \ldots, m$ and that this condition determines $a$ in $E^{\prime}$ and $c$ uniquely up to constant multiple. Moreover for any subset $J^{\prime}$ of $J$, we have

$$
\left(a,\left(e\left(J^{\prime}\right)-e\left(J-J^{\prime}\right)\right) / 2\right) \leqslant c .
$$

Since $\left(e\left(J_{\alpha}^{\prime}\right)-e\left(J-J_{\alpha}^{\prime}\right)\right)-\left(e\left(J^{\prime}\right)-e\left(J-J^{\prime}\right)\right)=e\left(J_{\alpha}^{\prime}\right)-e\left(J^{\prime}\right)$, we see that $\left(a, e\left(J^{\prime}\right)\right) \leqslant\left(a, e\left(J_{\alpha}^{\prime}\right)\right)$ for any subset $J^{\prime}$ of $J$, with the equality holding if $J^{\prime}=J_{\beta}^{\prime}$ for some $1 \leqslant \beta \leqslant m$. Let $c^{\prime}=\left(a, e\left(J_{1}^{\prime}\right)\right)=\cdots=\left(a, e\left(J_{m}^{\prime}\right)\right)$. Thus we have $\left(a, e\left(J^{\prime}\right)\right) \leqslant c^{\prime}$ for any subset $J^{\prime}$ of $J$ with the equality holding if $J^{\prime}=J_{\alpha}^{\prime}$ for some $\alpha$. We may assume without loss of generality. that $g=$ $\left\{J_{1}^{\prime}, \ldots, J_{m}^{\prime}\right\}$ is the set of all subsets $J^{\prime}$ of $J$ for which the equality holds.

LEMMA 5.3. Let $\left(a, e\left(J^{\prime}\right)\right) \leqslant c^{\prime}$ for any subset $J^{\prime}$ of $J$ with the equality holding if and only if $J^{\prime}$ belongs to a family $g$ of subsets. Then there exists $a$ decomposition

$$
J=J_{+} \amalg J_{0} \amalg J_{-}
$$

such that $\left(a, e_{j}\right)>0$ if $j$ is in $J_{+},\left(a, e_{j}\right)=0$ if $j$ is in $J_{0}$ and $\left(a, e_{j}\right)<0$ if $j$ is in $J_{-}$such that $g$ is exactly the family of subsets $J^{\prime}$ of $J$ satisfying $J_{+} \amalg J_{0} \supset J^{\prime}$ $J_{+}$.

Proof of Lemma 5.3. First of all, $g$ is closed under union and intersection. Indeed, for $J_{1}^{\prime}$ and $J_{2}^{\prime}$ in $\mathcal{G}$, we have

$$
2 c^{\prime}=\left(a, e\left(J_{1}^{\prime}\right)\right)+\left(a, e\left(J_{2}^{\prime}\right)\right)=\left(a, e\left(J_{1}^{\prime} \cup J_{2}^{\prime}\right)\right)+\left(a, e\left(J_{1}^{\prime} \cap J_{2}^{\prime}\right)\right)
$$

and each term is not greater than $c^{\prime}$ by assumption. Thus there exist the largest member $J_{3}^{\prime}$ and the smallest member $J_{4}^{\prime}$ in $g$. An element $j$ in $J$ is not in $J_{3}^{\prime}$ if and only if $\left(a, e_{j}\right)<0$. Indeed, if $j$ is not in $J_{3}^{\prime}$, then $\left(a, e_{j}\right)=\left(a, e_{j}\right)+$ $\left(a, e\left(J_{3}^{\prime}\right)\right)-c^{\prime}=\left(a, e\left(\{j\} \cup J_{3}^{\prime}\right)\right)-c^{\prime}<0$, and if $j$ is in $J_{3}^{\prime}$, then $\left(a, e_{j}\right)=$ $\left(a, e\left(J_{3}^{\prime}\right)\right)-\left(a, e\left(J_{3}^{\prime}-\{j\}\right)\right) \geqslant 0$. Similarly $j$ is in $J_{4}^{\prime}$ if and only if $\left(a, e_{j}\right)>0$. Indeed, if $j$ is in $J_{4}^{\prime}$, then $\left(a, e_{j}\right)=\left(a, e\left(J_{4}^{\prime}\right)\right)-\left(a, e\left(J_{4}^{\prime}-\{j\}\right)\right)>0$, and if $j$ is not in $J_{4}^{\prime}$, then $\left(a, e_{j}\right)=\left(a, e\left(J_{4}^{\prime} \cup\{j\}\right)\right)-\left(a, e\left(J_{4}^{\prime}\right)\right) \leqslant 0$. Thus we are done by putting $J_{+}=J_{4}^{\prime}, J_{-}=J-J_{3}^{\prime}$ and $J_{0}=J-J_{+} \amalg J_{-}$. 
Proof of Proposition 5.2 Continued. By Lemma 4.3 we see that $a \neq 0$ in $E^{\prime}$ should be determined up to constant multiple by the conditions $\left(a, e_{j}\right)>0$ for $j$ in $J_{+},\left(a, e_{j}\right)=0$ for $j$ in $J_{0}$ and $\left(a, e_{j}\right)<0$ for $j$ in $J_{-}$. Consider the spanning subgraph $\Gamma^{\prime \prime}=\left\{I, J^{\prime \prime}\right\}$ with $J^{\prime \prime}=J_{+} \amalg J_{-}$. By the unicity of $a$ up to scalar multiplication, we conclude easily that $H_{1}\left(\Gamma^{\prime \prime}, \mathbf{R}\right)$ is one dimensional, hence we may assume that $a$ is an elementary cycle $\gamma$ by Lemma 4.6. Since for a subset $J^{\prime}$ of $J$ we have

$\left(\gamma,\left(e\left(J^{\prime}\right)-e\left(J-J^{\prime}\right)\right) / 2\right)=\left(\left|J_{+}\right|+\left|J_{-}\right|\right) / 2-\left|J_{+} \cap\left(J-J^{\prime}\right)\right|-\left|J_{-} \cap J^{\prime}\right|$, its maximal value $(\gamma, \gamma) / 2=\left(\left|J_{+}\right|+\left|J_{-}\right|\right) / 2$ is attained for $J_{+} \subset J^{\prime} \subset$ $J_{+} \amalg J_{0}$. Hence $c=(\gamma, \gamma) / 2$ and the codimension one face is determined by the hyperplane

$$
H=\left\{x \in E^{\prime} ;(x, \gamma)=(\gamma, \gamma) / 2\right\} \text {. }
$$

The converse is obvious. To prove (2), we proceed as above and reduce ourselves to the situation where $a \neq 0$ in $E^{\prime \prime}$ is determined up to scalar multiplication by the conditions $\left(a, e_{j}\right)>0$ for $j$ in $J_{+},\left(a, e_{j}\right)=0$ for $j$ in $J_{0}$ and $\left(a, e_{j}\right)<0$ for $j$ in $J_{-}$. Consider the spanning subgraph $\Gamma_{0}=\left\{I, J_{0}\right\}$ and the projection $P_{0}: C_{1}(\Gamma, \mathbf{R}) \rightarrow C_{1}\left(\Gamma_{0}, \mathbf{R}\right)$ defined by $P_{0}\left(e_{j}\right)=0$ if $j$ is not in $J_{0}$, while $P_{0}\left(e_{j}\right)=e_{j}$ if $j$ is in $J_{0}$. By the unicity of $a$ up to scalar multiplication, we conclude easily that $\operatorname{ker}\left(P_{0}\right) \cap \delta C_{0}(\Gamma, \mathbf{R})$ is one dimensional, hence we may assume that $a$ is an elementary cocycle $\omega$ by Lemma 4.7. Again for a subset $J^{\prime}$ of $J$ we have

$$
\left(\omega,\left(e\left(J^{\prime}\right)-e\left(J-J^{\prime}\right)\right) / 2\right)=(\omega, \omega) / 2-\left|J_{+} \cap\left(J-J^{\prime}\right)\right|-\left|J_{-} \cap J^{\prime}\right|,
$$

hence its maximal value is attained for $J_{+} \subset J^{\prime} \subset J_{+} \cup J_{0}$. Thus the codimension one face is determined by the hyperplane

$$
H=\left\{x \in E^{\prime \prime} ;(x, \omega)=(\omega, \omega) / 2\right\} \text {. }
$$

The converse is again obvious.

COROLlaRY 5.4. For an element of $E^{\prime \prime}$ of the form

$$
\psi=\pi^{\prime \prime}(\xi+e(J) / 2)
$$

for $\xi$ in $\Lambda$, the Namikawa decomposition $\operatorname{Del}_{\psi}\left(E^{\prime} ; E, \Lambda\right)$ coincides with the translation by $\pi^{\prime}(\xi+e(J) / 2)$ of the Voronoi decomposition $\operatorname{Vor}\left(E^{\prime}, \Lambda \cap E^{\prime}\right)$, i.e.

$$
\operatorname{Del}_{\pi^{\prime \prime}(\xi+e(J) / 2)}\left(E^{\prime} ; E, \Lambda\right)=\pi^{\prime}(\xi+e(J) / 2)+\operatorname{Vor}\left(E^{\prime}, \Lambda \cap E^{\prime}\right) .
$$

Proof. By our explicit description of $\operatorname{Vor}(\Gamma)=\operatorname{Vor}(E, \Lambda)$, we see that $u=\xi+e(J) / 2$ is a 0 -dimensional Voronoi polyhedron. Thus $u^{\prime}=u+V_{J}(0)$ and $\pi^{\prime}\left(u^{*}\right)=\pi^{\prime}(u)+\pi^{\prime}\left(V_{J}(0)\right)$ is in $\operatorname{Del}_{\psi}\left(E^{\prime} ; E, \Lambda\right)$. Since this latter is invariant under the translation by elements of $\Lambda \cap E^{\prime}$, we are done by Proposition 5.2 (1).

REMARK. Compare this result with the following "dual" version due to 
Mumford which was used by Namikawa $[27, \S 18]$ for his compactification of the generalized Jacobian variety of a stable curve.

Proposition 5.5 (Mumford). Let $E, \Lambda, E^{\prime}$ and $E^{\prime \prime}$ be as before defined for a graph $\Gamma$. Then

(1) the polyhedral decomposition of $E^{\prime}$

$$
\left\{D \cap E^{\prime} ; D \in \operatorname{Del}(E, \Lambda) \text { with } \operatorname{rel} \operatorname{int}(D) \cap E^{\prime} \neq \varnothing\right\}
$$

induced by the Delony decomposition of $E$ coincides with the Delony decomposition $\operatorname{Del}\left(E^{\prime}, \Lambda \cap E^{\prime}\right)$ and

(2) the polyhedral decomposition of $E^{\prime \prime}$

$$
\left\{D \cap E^{\prime \prime} ; D \in \operatorname{Del}(E, \Lambda) \text { with } \operatorname{rel} \text {.int }(D) \cap E^{\prime \prime} \neq \varnothing\right\}
$$

induced by the Delony decomposition of $E$ coincides with the Delony decomposition $\operatorname{Del}\left(E^{\prime \prime}, \Lambda \cap E^{\prime \prime}\right)$.

Proof. For $j$ in $J$ consider the linear form $f_{j}(x)=\left(e_{j}, x\right)$ on $E$. Then $\left\{f_{j}\right.$; $j \in J\}$ is the basis of $\Lambda^{-}$dual to the basis $\left\{e_{j} ; j \in J\right\}$ of $\Lambda$. The metric on $E$ can be written as

$$
\|x\|^{2}=\sum_{j \in J} f_{j}(x)^{2}
$$

Consider the restriction of those to subspaces $E^{\prime}$ and $E^{\prime \prime}$, and their lattices $\Lambda \cap E^{\prime}$ and $\Lambda \cap E^{\prime \prime}$. It is a standard fact in graph theory that $\left\{f_{j} \mid E^{\prime} ; j \in J\right\}$ and $\left\{f_{j} \mid E^{\prime \prime} ; j \in J\right\}$ are totally unimodular subsets of $\left(\Lambda \cap E^{\prime}\right)^{-}$and $(\Lambda \cap$ $\left.E^{\prime \prime}\right)^{-}$, respectively. Indeed, for a subset $J^{\prime}$ of $J$, the set $\left\{f_{j} \mid E^{\prime} ; j \in J^{\prime}\right\}$ is an R-basis of $(E)^{-}$if and only if there exists a spanning forest $\{I, T\}$ of $\Gamma$ such that $J^{\prime}=J-T$, hence it is a Z-basis of $\left(\Lambda \cap E^{\prime}\right)^{-}$. Similarly, the set $\left\{f_{j} \mid E^{\prime \prime}\right.$; $\left.j \in J^{\prime}\right\}$ is an $\mathbf{R}$-basis of $\left(E^{\prime \prime}\right)^{-}$if and only if there exists a spanning forest $\{I, T\}$ such that $J^{\prime}=T$, hence it is a Z-basis of $\left(\Lambda \cap E^{\prime \prime}\right)^{-}$. The rest of the proposition follows immediately from Corollary 3.2 applied to $F=E^{\prime}$ and $F=E^{\prime \prime}$.

Remark. Although we do not need it later, we can show that there is a surjective map from the set of "flags"

$$
I_{1} \subset I_{2} \subset \cdots \subset I_{n} \subset I
$$

(i.e. $\left|I_{\alpha}\right|=\alpha$ and $\left.|I|=n+1\right)$ to the set of vertices of $\pi^{\prime \prime}\left(V_{J}(0)\right)$. This fits in nicely with the following known result valid for Voronoi decompositions in general: The number of vertices of a top-dimensional Voronoi polyhedron in an $n$-dimensional space is not greater than $(n+1)$ ! (cf. Voronoi [34]).

6. Namikawa decompositions for a graph. We consider now Namikawa decompositions for a graph $\Gamma=\{I, J\}$. For later purpose, it is more convenient to express Namikawa decompositions on $H^{1}(\Gamma, \mathbf{R})$ which is isomorphic via $\rho$ to $E^{\prime}$. It is also more convenient to take the parameters in 
$\partial C_{1}(\Gamma, \mathbf{R})$ which is isomorphic via $\partial$ to $E^{\prime \prime}$, i.e. for an element $\phi$ in $\partial C_{1}(T, \mathbf{R})$ there exists a unique element $\psi$ in $E^{\prime \prime}$ such that $\phi=\partial \psi$. Recall that we have $\partial \circ \pi^{\prime \prime}=\partial$.

Definition. Let $\phi$ be in $\partial C_{1}(\Gamma, \mathbf{R})$.

(i) A Delony polyhedron $D$ in $K(\Gamma)$ is called $\phi$-semistable if $\partial D^{*} \exists \phi$. We denote by $K_{\phi}(\Gamma)$ the set of $\phi$-semistable Delony polyhedra.

(ii) We denote by $K_{\phi}^{0}(T)$ the set of Delony polyhedra $D$ in $K(T)$ such that $\partial$ (rel.int $\left.D^{*}\right) \ni \phi$.

(iii) A Delony polyhedron $D$ in $K(\Gamma)$ is called $\phi$-stable if $\partial$ (rel.int $\left.D^{*}\right) \ni \phi$ and $\operatorname{dim} \partial D^{*}=\operatorname{dim} \partial C_{1}(\Gamma, \mathbf{R})$. We denote by $K_{\phi \text {-stable }}(\Gamma)$ the set of $\phi$-stable Delony polyhedra.

(iv) We denote by $\operatorname{Del}_{\phi}\left(H^{1}(T, \mathbf{R})\right)$ the set of polyhedra in $H^{1}(T, \mathbf{R})$ of the form $\rho(D)$ for $D$ in $K_{\phi}^{0}(T)$.

Note that $K_{\phi}(\Gamma)$ and $K_{\phi-s t a b l e}(\Gamma)$ contain faces of each of their members (cf. Corollary 2.2), while $K_{\phi}^{0}(\Gamma)$ does not. $K_{\phi}(\Gamma)$ consists of faces of members of $K_{\phi}^{0}(\Gamma)$. We have $K_{\phi \text {-stable }}(\Gamma) \subset K_{\phi}^{0}(\Gamma) \subset K_{\phi}(\Gamma)$. As we saw in $\$ 2$, we have a surjective map

$$
\mu: K_{\phi}(\Gamma) \rightarrow K_{\phi}^{0}(\Gamma)
$$

quasi-inverse to the inclusion. $D_{1}$ and $D_{2}$ in $K_{\phi}(\Gamma)$ are said to be $\phi$-equivalent if $\mu\left(D_{1}\right)=\mu\left(D_{2}\right) . D \in K_{\phi}(T)$ is in $K_{\phi-\text {-stable }}(T)$ if and only if $D$ is in $K_{\phi}^{0}(T)$ and is the unique element $\phi$-equivalent to it (cf. Proposition 2.1). We have

$$
K_{\phi}(\Gamma) / \phi \text {-equiv. } \stackrel{\mu}{\sim} K_{\phi}^{0}(\Gamma) \stackrel{\rho}{\sim} \operatorname{Del}_{\phi}\left(H^{1}(\Gamma, \mathbf{R})\right) \text {. }
$$

We are going to analyze $\phi$-equivalence further at the end of $\S 12$ (Lemma 12.16).

We can interpret Proposition 1.6 as follows:

Proposimion 6.1. For $\phi$ in $\partial C_{1}(\Gamma, \mathbf{R})$, the set $\operatorname{Del}_{\phi}\left(H^{1}(\Gamma, \mathbf{R})\right)$ of polyhedra $\rho(D)$ for $D$ in $K_{\phi}^{0}(\Gamma)$ is a polyhedral decomposition, called the Namikawa decomposition, of $H^{1}(\Gamma, \mathbf{R})$ by bounded polyhedra invariant under the translation by elements of $\rho\left(H_{1}(\Gamma, \mathbf{Z})\right)$ with $\operatorname{Del}_{\phi}\left(H^{1}(\Gamma, \mathbf{R})\right) / \rho\left(H_{1}(\Gamma, Z)\right)$ finite. Moreover the set $\operatorname{Sk}^{0}\left(\operatorname{Del}_{\phi}\left(H^{1}(\Gamma, \mathbf{R})\right)\right)$ of 0 -dimensional polyhedra is a subset of $H^{1}(\Gamma, Z)$.

COROllaRY 6.2. Let $u$ be an element of $C_{1}(\Gamma, \mathbf{R})$ with $u-e(J) / 2$ in $C_{1}(\Gamma, \mathbf{Z})$. Then $\operatorname{Del}_{\partial u}\left(H^{1}(\Gamma, \mathbf{R})\right)$ is the translation by $\rho(u)$ of the image under $\rho$ of the Voronoi decomposition $\operatorname{Vor}\left(H_{1}(\Gamma, \mathbf{R}), H_{1}(\Gamma, \mathbf{Z})\right)$, i.e. for $\xi \in C_{1}(\Gamma, Z)$ we have

$\operatorname{Del}_{\partial(\xi+e(J) / 2)}\left(H^{1}(\Gamma, \mathbf{R})\right)=\rho(\xi+e(J) / 2)+\rho\left(\operatorname{Vor}\left(H_{1}(\Gamma, \mathbf{R}), H_{1}(\Gamma, \mathbf{Z})\right)\right)$.

This is nothing but a re-interpretation of Corollary 5.4 . 
COROLlaRY 6.3. The translates by elements of $\partial C_{1}(\Gamma, \mathbf{Z})$ of the faces of $\partial V_{J}(0)$ is a polyhedral decomposition of $\partial C_{1}(\Gamma, \mathbf{R})$, the image under $\partial$ of the Voronoi decomposition $\operatorname{Vor}\left(\delta C_{0}(\Gamma, \mathbf{R}), \delta C_{0}(\Gamma, \mathbf{Z})\right)$. Moreover, we have

$$
\begin{aligned}
\partial V_{J}(0)=\left\{x \in \partial C_{1}(\Gamma, \mathbf{R}) \mid\left[x, v\left(I^{\prime}\right)\right] \leqslant\left(\delta v\left(I^{\prime}\right), \delta v\left(I^{\prime}\right)\right) / 2\right. \\
\left.\quad \text { for all subsets } I^{\prime} \text { of } I\right\} .
\end{aligned}
$$

It is sufficient to take only those $I^{\prime}$ for which $\delta v\left(I^{\prime}\right)$ are elementary.

This is a re-interpretation of Proposition 5.2 (2).

REMARK. We need to know below the relative interior of $\partial\left(V_{J}(0)\right)$. Since we have $\operatorname{ker}(\delta)=H^{0}(\Gamma, \mathbf{Z})=\bigoplus_{1 \leqslant \alpha<c} \mathrm{Z} v\left(I_{\alpha}\right)$ by $\$ 4$, we conclude that rel.int $\left(\partial\left(V_{J}(0)\right)\right)$ consists of $x$ in $\partial C_{1}(T, \mathbf{R})$ which satisfy

$$
\left[x, v\left(I^{\prime}\right)\right]<\left(\delta v\left(I^{\prime}\right), \delta v\left(I^{\prime}\right)\right) / 2
$$

for any subset $I^{\prime}$ of $I$ which is not the set of vertices in a union of connected components. It is again sufficient to take only those $I^{\prime}$ for which $\delta v\left(I^{\prime}\right)$ are elementary.

In the case of a graph we can determine $\phi$-semistability and $\phi$-stability of Delony polyhedra more easily in the following way:

Proposition 6.4. Let $D$ be a Delony polyhedron in $K(\Gamma)$, and let $\phi$ be in $\partial C_{1}(\Gamma, \mathbf{R})$. Then the following are equivalent:

(1) $D$ is $\phi$-semistable, i.e. $D$ is in $K_{\phi}(\Gamma)$.

(2) $\partial b(D)-\phi \in \partial\left(V_{J}(0)\right)$, where $J^{\prime}=J-\operatorname{Supp}(D)$.

(3) $\partial D \subset \phi+\partial\left(V_{J}(0)\right)$.

Proof. Let $J^{\prime \prime}=\operatorname{Supp}(D)$ and $J^{\prime}=J-J^{\prime \prime}$. Then for $b=b(D)$, we have $D=b+V_{J^{\prime \prime}}(0)$ and $D^{*}=b+V_{J^{\prime}}(0)$ by Proposition 5.1. By definition, $D$ is $\phi$-semistable if and only if $\partial D^{*} \ni \phi$. Hence (1) is obviously equivalent to (2). (2) implies (3), since

$$
\partial D=\partial\left(b+V_{J^{\prime \prime}}(0)\right) \subset \phi+\partial\left(V_{J^{\prime}}(0)\right)+\partial\left(V_{J^{\prime \prime}}(0)\right)=\phi+\partial\left(V_{J}(0)\right) \text {. }
$$

Finally (3) implies (2). Indeed, we have $\delta v\left(I^{\prime}\right)=\delta_{J}, v\left(I^{\prime}\right)+$ $\sum_{j^{\prime \prime} \in J^{\prime \prime}}\left[v\left(I^{\prime}\right), \partial e_{j^{\prime \prime}}\right] e_{j^{m}}$, hence

$$
\left(\delta v\left(I^{\prime}\right), \delta v\left(I^{\prime}\right)\right)=\left(\delta_{J^{\prime}} v\left(I^{\prime}\right), \delta_{J^{\prime}} v\left(I^{\prime}\right)\right)+\sum_{j^{\prime \prime} \in J^{\prime \prime}}\left[v\left(I^{\prime}\right), \partial e_{j^{\prime \prime}}\right]^{2}
$$

Thus for $y=b+\sum_{j^{\prime \prime} \in J^{\prime \prime}} \lambda_{j^{m}} e_{j^{\prime \prime}}$ in $D$ with $\left|\lambda_{j^{\prime \prime}}\right| \leqslant 1 / 2$, we have

$$
\begin{aligned}
{\left[\partial b-\phi, v\left(I^{\prime}\right)\right]-\left(\delta_{J^{\prime}} v\left(I^{\prime}\right), \delta_{J^{\prime}} v\left(I^{\prime}\right)\right) / 2 } \\
=\left[\partial y-\phi, v\left(I^{\prime}\right)\right]-\left(\delta v\left(I^{\prime}\right), \delta v\left(I^{\prime}\right)\right) / 2 \\
\quad+\sum_{j^{\prime \prime} \in J^{\prime \prime}}\left(\left[v\left(I^{\prime}\right), \partial e_{j^{\prime \prime}}\right]^{2} / 2-\lambda_{j^{\prime \prime}}\left[v\left(I^{\prime}\right), \partial e_{j^{\prime \prime}}\right]\right) .
\end{aligned}
$$


The first half of the right-hand side is nonpositive by (3) and Corollary 6.3, and the second half attains its minimal value 0 for $\lambda_{j^{\prime \prime}}=\left[v\left(I^{\prime}\right), \partial e_{j^{n}}\right] / 2$, since $\left[v\left(I^{\prime}\right), \partial e_{j^{\prime \prime}}\right]=0$ or \pm 1 . Hence $\left[\partial b-\phi, v\left(I^{\prime}\right)\right] \leqslant\left(\delta_{J}, v\left(I^{\prime}\right), \delta_{J^{\prime}} v\left(I^{\prime}\right)\right) / 2$, and we get (2) by applying Corollary 6.3 to the graph $\left\{I, J^{\prime}\right\}$. Q.E.D.

Proposition 6.5. Let $D$ and $\phi$ be as above. Then the following are equivalent:

(1) $D$ is in $K_{\phi}^{0}(\Gamma)$.

$\left(1^{\prime}\right) \partial b(D)-\phi \in \operatorname{rel} . i n t\left(\partial V_{J}(0)\right)$ with $J^{\prime}=J-\operatorname{Supp}(D)$.

(2) For any $y$ in $D$, we have

$$
\left[\partial y-\phi, v\left(I^{\prime}\right)\right] \leqslant\left(\delta v\left(I^{\prime}\right), \delta v\left(I^{\prime}\right)\right) / 2
$$

for any subset $I^{\prime}$ of $I$, with the equality holding for some $y$ in $D$ only if $I^{\prime}$ is the set of vertices in a union of connected components in the graph $\left\{I, J^{\prime}\right\}$ with $J^{\prime}=J-\operatorname{Supp}(D)$.

ProOF. (1) and $\left(1^{\prime}\right)$ are equivalent by definition. By the remark after Corollary 6.3 applied to the graph $\left\{I, J^{\prime}\right\}$, we see that $\left(1^{\prime}\right)$ is equivalent to $\left(1^{\prime \prime}\right)$ $\left[\partial b(D)-\phi, v\left(I^{\prime}\right)\right] \leqslant\left(\delta_{J}, v\left(I^{\prime}\right), \delta_{J}, v\left(I^{\prime}\right)\right) / 2$ for any subset $I^{\prime}$ of $I$ with the equality holding (if and) only if $I^{\prime}$ is the set of vertices in a union of connected components of $\left\{I, J^{\prime}\right\}$. For $y=b+\sum_{j^{\prime \prime} \in J^{\prime \prime}} \lambda_{j^{\prime \prime}} e_{j^{\prime \prime}}$ in $D$ with $\left|\lambda_{j^{\prime \prime}}\right| \leqslant$ 1/2, we have as before $A=B(y)+C(y)$, where

$$
\begin{aligned}
A & =\left[\partial b-\phi, v\left(I^{\prime}\right)\right]-\left(\delta_{J^{\prime}} v\left(I^{\prime}\right), \delta_{J^{\prime}} v\left(I^{\prime}\right)\right) / 2 \\
B(y) & =\left[\partial y-\phi, v\left(I^{\prime}\right)\right]-\left(\delta v\left(I^{\prime}\right), \delta v\left(I^{\prime}\right)\right) / 2 \text { and } \\
C(y) & =\sum_{j^{\prime \prime} \in J^{\prime \prime}}\left(\left[v\left(I^{\prime}\right), \partial e_{j^{\prime \prime}}\right]^{2} / 2-\lambda_{j^{\prime \prime}}\left[v\left(I^{\prime}\right), \partial e_{j^{\prime \prime}}\right]\right) .
\end{aligned}
$$

We have $C(y) \geqslant 0$ with the equality attained for $y=y_{0}$ with $\lambda_{j^{\mu}}=$ $\left[v\left(I^{\prime}\right), \partial e_{j^{\prime \prime}}\right] / 2$

( $\left.1^{\prime \prime}\right)$ implies (2), since $0 \geqslant A \geqslant B(y)$ for all $y$ and since $B(y)=0$ only if $A=0$ and $C(y)=0$. On the other hand (2) implies (1"), since $A=B\left(y_{0}\right) \leqslant$ 0 , and since $A=0$ only if $B\left(y_{0}\right)=0$.

Proposition 6.6. Let $D$ and $\phi$ be as above. Then the following are equivalent:

(1) $D$ is $\phi$-stable, i.e. $D$ is in $K_{\phi-s t a b l e}(\Gamma)$.

(1') $\partial b(D)-\phi \in \operatorname{rel} . i n t\left(\partial V_{J}(0)\right)$ with $J^{\prime}=J-\operatorname{Supp}(D)$ containing the set of edges of a spanning forest of $\Gamma$.

(2) $\partial D \subset \phi+\operatorname{rel}$ int $\left(\partial V_{J}(0)\right)$.

(3) $D$ is in $K_{\phi}^{0}(\Gamma)$ and $\rho$ induces a bijection $D \stackrel{\sim}{\rightarrow} \rho(D)$.

(3') $D$ is in $K_{\phi}^{0}(\Gamma)$ and $\operatorname{dim} D=\operatorname{dim} \rho(D)$.

(4) $D$ is the unique element equivalent to it in the equivalence relation defined 
by

as in $\$ 2$.

$$
\mu: K_{\phi}(\Gamma) \rightarrow K_{\phi}^{0}(\Gamma)
$$

Proof. The equivalence of (1), (3), (3') and (4) follows from Proposition 2.1. (1) and $\left(1^{\prime}\right)$ are equivalent, since $D^{*}=b(D)+V_{J^{\prime}}(0)$ and since $\operatorname{dim} \partial V_{J}(0)=\operatorname{dim} \partial C_{1}(\Gamma, \mathbf{R})$ if and only if $J^{\prime}$ contains the set of edges of a spanning forest of $\Gamma$. On the other hand, $J^{\prime}$ contains the set of edges of a spanning forest of $\Gamma$ if and only if the connected components of $\Gamma$ remain connected in $\left\{I, J^{\prime}\right\}$. Thus in view of the previous proposition $\left(1^{\prime}\right)$ is equivalent to: (2') For any $y$ in $D$ we have

$$
\left[\partial y-\phi, v\left(I^{\prime}\right)\right]<\left(\delta v\left(I^{\prime}\right), \delta v\left(I^{\prime}\right)\right) / 2
$$

for any subset $I^{\prime}$ of $I$ with the equality holding for some $y$ in $D$ if and only if $I^{\prime}$ is the set of vertices in a union of connected components of $\Gamma$. By the remark after Corollary 6.3, (2') is obviously equivalent to (2). Q.E.D.

Remark. Proposition 6.4 (3) and Proposition 6.6 (2) are very powerful and convenient criteria for the semistability and stability of a Delony polyhedron. We cannot expect to have similar criteria in general when $E$ need not come from a graph. See, for instance, the last example in $\$ 1$. These criteria were our original definition of semistability and stability to which we were led by algebro-geometric considerations we make in the next chapter.

7. Relations among Namikawa decompositions for a graph. In this section, we study more closely the polyhedral decomposition $\operatorname{Par}\left(E^{\prime \prime}\right)$ introduced at the end of $\$ 2$, when $E$ comes from a graph. Thus as in $\$ 2$, we can compare Namikawa decompositions $\operatorname{Del}_{\phi}\left(H^{1}(T, \mathbf{R})\right)$ for different values of the parameter $\phi$. We interpret $\operatorname{Par}\left(E^{\prime \prime}\right)$ in terms of an arrangement of hyperplanes which we introduced in $\$ 3$ and which is much easier to compute.

Let $\Gamma=\{I, J\}$ be a graph and let $E, E^{\prime}, E^{\prime \prime}$ and $\Lambda$ be as in $\S 4$. Furthermore, we let

$$
F=\partial C_{1}(\Gamma, \mathbf{R}) \text { and } G=\partial C_{1}(\Gamma, \mathbf{Z}) .
$$

Note that $\partial$ induces an isomorphism $\partial: E^{\prime \prime} \rightarrow F$ which sends $\pi^{\prime \prime}(\Lambda)$ isomorphically onto $G$. We try to express $\operatorname{Par}\left(E^{\prime \prime}\right)$ in a more computable form on $F$, as an arrangement of hyperplanes.

Defintion. We call a subset $I^{\prime}$ of $I$ elementary when $\delta v\left(I^{\prime}\right)$ is an elementary cocycle (cf. Lemma 4.7). For an elementary subset $I^{\prime}$ of $I$, we denote by $f_{I^{\prime}}$ the element of $\check{G}=\operatorname{Hom}_{\mathrm{Z}}(G, Z)$ defined by $f_{I^{\prime}}(g)=\left[v\left(I^{\prime}\right), g\right]$ for $g$ in $G$, where [ , ] is the pairing, on $C_{0}(\Gamma, \mathbf{Z})$.

Definition. We denote by $\operatorname{Arr}(T)$ the arrangement of hyperplanes in $F=\partial C_{1}(\Gamma, \mathbf{R})$ defined by

$$
\operatorname{Arr}(\Gamma)=\operatorname{Arr}\left(F,\left\{f_{I^{\prime}} ; I^{\prime} \text { elementary subsets of } I\right\}\right) .
$$


THEOREM 7.1. For a graph $\Gamma$, we have

$$
\partial\left(\operatorname{Par}\left(E^{\prime \prime}\right)\right)=\partial e(J) / 2+\operatorname{Arr}(\Gamma)
$$

which is invariant under the translation action of $\partial C_{1}(\Gamma, Z)$ with the quotient finite. Moreover, $\operatorname{Sk}^{0}(\operatorname{Arr}(\Gamma)) \supset \partial C_{1}(\Gamma, \mathbf{Z})$.

COROLlaRY 7.2. (i) For $A$ in $\operatorname{Arr}(\Gamma)$, the sets $K_{\phi}(\Gamma), K_{\phi}^{0}(\Gamma)$ and $K_{\phi-s t a b l e}(\Gamma)$ stay the same as long as $\phi$ is in $\partial e(J) / 2+\operatorname{rel} \operatorname{int}(A)$.

(ii) For $A$ in $\operatorname{Arr}(\Gamma)$, the Namikawa decomposition $\operatorname{Del}_{\phi}\left(H^{1}(T, \mathbf{R})\right)$ stays the same as long as $\phi$ is in $\partial e(J) / 2+\operatorname{rel} \operatorname{int}(A)+\partial \delta C_{0}(\Gamma, \mathbf{Z})$.

(iii) For $\xi$ in $C_{1}(\Gamma, Z)$, and $\phi$ in $\partial C_{1}(\Gamma, \mathbf{R})$, we have $K_{\phi+\partial \xi}(\Gamma)=K_{\phi}(\Gamma)+\xi$, $K_{\phi+\partial \xi}^{0}(\Gamma)=K_{\phi}^{0}(\Gamma)+\xi, \quad K_{\phi+\partial \xi \text {-stable }}(\Gamma)=K_{\phi \text {-stable }}(\Gamma)+\xi$ and $\operatorname{Del}_{\phi+\partial \xi}\left(H^{1}(\Gamma, \mathbf{R})\right)=\operatorname{Del}_{\phi}\left(H^{1}(\Gamma, \mathbf{R})\right)+\rho(\xi)$.

(iv) Let $\bar{A}$ be a face of $A$ in $\operatorname{Arr}(\Gamma)$. For $\phi$ in $\partial e(J) / 2+\operatorname{rel}$.int $(A)$ and $\bar{\phi}$ in $\partial e(J) / 2+\operatorname{rel} . i n t(\bar{A})$, the Namikawa decomposition $\operatorname{Del}_{\phi}\left(H^{1}(T, \mathbf{R})\right)$ is a subdivision of $\operatorname{Del}_{\bar{\phi}}\left(H^{1}(T, \mathbf{R})\right)$.

CORÓlLARY 7.3. For a graph $\Gamma$, there are only a finite number of different Namikawa decompositions of $H^{1}(\Gamma, \mathbf{R})$, the number being bounded by the cardinality of

$$
\operatorname{Arr}(\Gamma) / \partial \delta C_{0}(\Gamma, \mathbf{Z}) \text {. }
$$

Among them there are those which differ only by the translation by elements of $H^{1}(\Gamma, \mathbf{Z})$.

These are just a re-interpretation of Proposition 2.3 and Corollary 2.4.

Proof of TheOREM 7.1. Since $\partial$ induces an isomorphism $\partial: E^{\prime \prime} \rightarrow F$, it is enough to show that

$$
\operatorname{Par}\left(E^{\prime \prime}\right)=\pi^{\prime \prime}(e(J) / 2)+\operatorname{Arr}\left(E^{\prime \prime},\left\{\omega_{1}, \ldots, \omega_{a}\right\}\right)
$$

where $\left\{\omega_{1}, \ldots, \omega_{a}\right\}$ is the set of all the elementary cocycles for $\Gamma$ and we identify $\omega_{\alpha}$ with the linear form $\xi \mapsto\left(\omega_{\alpha}, \xi\right)$ belonging to $\left(\Lambda \cap E^{\prime \prime}\right)^{-}$.

Let $\psi$ be an element of $E^{\prime \prime}$. Then by the definition given in $\S 2$, we have $P(\psi)=\cap \pi^{\prime \prime}(V)$ where $V$ runs over all the Voronoi polyhedra in $\operatorname{Vor}(\Gamma)$ with $V^{*}$ in $K_{\partial \psi}(\Gamma)$.

LEMMA 7.4. Let $V$ be in $\operatorname{Vor}(\Gamma)$ and let $V^{*}=\left\langle\xi_{0}, \ldots, \xi_{r}\right\rangle$. Then we have

$$
\pi^{\prime \prime}(V)=\pi^{\prime \prime}\left(V\left(\xi_{0}\right)\right) \cap \cdots \cap \pi^{\prime \prime}\left(V\left(\xi_{r}\right)\right) .
$$

In particular for $\psi$ in $E^{\prime \prime}$, we have

$$
P(\psi)=\bigcap \pi^{\prime \prime}(V(\xi))
$$

with $V(\xi)$ running over all the top-dimensional Voronoi polyhedra in $\operatorname{Vor}(\Gamma)$ with $\pi^{\prime \prime}(V(\xi)) \ni \psi$, i.e. $\xi$ in $\Lambda \cap K_{\partial \psi}(\Gamma)$. 
Proof of LemMa 7.4. Obviously we have $V=V\left(\xi_{0}\right) \cap \cdots \cap V\left(\xi_{r}\right)$. Thus $\pi^{\prime \prime}(V)$ is contained in the right-hand side. Let $\psi$ be an element of the right-hand side. Thus $\xi_{\alpha}=V\left(\xi_{\alpha}\right)^{*}$ is contained in $K_{\partial \psi}(\Gamma)$ for $0 \leqslant \alpha \leqslant r$. Hence by Corollary $6.4(3)$, we see that $\partial \xi_{\alpha}$ is contained in $\partial \psi+\partial\left(V_{J}(0)\right)$ for $0 \leqslant \alpha \leqslant r$. But since this set is convex, we conclude that $\partial V^{*}=$ $\left\langle\partial \xi_{0}, \ldots, \partial \xi_{r}\right\rangle$ is contained in this set. Thus again by Corollary 6.4, we see that $V^{*}$ is in $K_{\partial \psi}(\Gamma)$, i.e. $\pi^{\prime \prime}(V) \ni \psi$.

PROOF OF THEOREM 7.1 CONTINUED. From our concrete description of the Voronoi decomposition $\operatorname{Vor}(\Gamma)$ and the Delony decomposition $K(T)$ of $E=$ $C_{1}(\Gamma, \mathbf{R})$ in Proposition 5.1, we see easily that they differ only by the translation by $e(J) / 2$, i.e.

$$
\operatorname{Vor}(\Gamma)=e(J) / 2+K(\Gamma)
$$

For simplicity, let us denote $\tilde{P}(\tilde{\psi})=P(\psi)-\pi^{\prime \prime}(e(J) / 2)$, where $\tilde{\psi}=\psi-$ $\pi^{\prime \prime}(e(J) / 2)$. Then by the above lemma we see that

$$
\tilde{P}(\tilde{\psi})=\bigcap \pi^{\prime \prime}\left(D_{1}\right)=\bigcap \pi^{\prime \prime}\left(D_{2}\right),
$$

where $D_{1}$ runs over all polyhedra in $K(\Gamma)$ with $\pi^{\prime \prime}\left(D_{1}\right) \ni \psi$, and $D_{2}$ runs over all top-dimensional polyhedra in $K(\Gamma)$ with $\pi^{\prime \prime}\left(D_{2}\right) \ni \tilde{\psi}$. Consider the intersection $\cap \pi^{\prime \prime}(D)$ where $D$ runs over all polyhedra in $K(\Gamma)$ with $\pi^{\prime \prime}(D) \ni \tilde{\psi}$ and moreover, with $\operatorname{Supp}(D)$ contained in the set of edges in a spanning forest of $\Gamma$. Then obviously this intersection contains $\cap \pi^{\prime \prime}\left(D_{1}\right)$ and is contained in $\cap \pi^{\prime \prime}\left(D_{2}\right)$. Thus we conclude that

$$
\tilde{P}(\tilde{\psi})=\bigcap_{T} \bigcap_{D} \pi^{\prime \prime}(D)
$$

where $\{I, T\}$ runs over all spanning forests of $\Gamma$ and with $D$ running over all polyhedra $D$ in $K(\Gamma)$ with $\pi^{\prime \prime}(D) \ni \tilde{\psi}$ and $\operatorname{Supp}(D)=T$. Hence our theorem follows from the following whose proof is left to the reader.

LEMMA 7.5. Let $\{I, T\}$ be a spanning forest of $\Gamma$. Then

$$
\left\{\pi^{\prime \prime}(D) ; D \in K(\Gamma), \operatorname{Supp}(D) \subset T\right\}
$$

is a polyhedral decomposition of $E^{\prime \prime}$, which coincides with the arrangement of hyperplanes

$$
\operatorname{Arr}\left(E^{\prime \prime},\left\{\omega_{T, t} ; t \in T\right\}\right)
$$

where $\left\{\omega_{T, t} ; t \in T\right\}$ is the Z-basis of $\Lambda \cap E^{\prime \prime}$ consisting of elementary cocycles dual to the Z-basis $\left\{\pi^{\prime \prime}\left(e_{t}\right) ; t \in T\right\}$ of $\pi^{\prime \prime}(\Lambda)$ with respect to the pairing (, ) which we introduced before Lemma 4.6.

Recall that for $\phi$ in $\partial C_{1}(\Gamma, \mathbf{R})$ we have

$$
K_{\phi \text {-stable }}(\Gamma) \subset K_{\phi}^{0}(\Gamma) \subset K_{\phi}(\Gamma)
$$


and an isomorphism

$$
\rho: K_{\phi}^{0}(\Gamma) \stackrel{\sim}{\rightarrow} \operatorname{Del}_{\phi}\left(H^{1}(\Gamma, \mathbf{R})\right) .
$$

By Corollary 6.3, we see that $\partial^{-1}\left(\phi+\partial V_{J}(0)\right)$ is a fundamental domain of $E=C_{1}(\Gamma, \mathbf{R})$ with respect to $\Lambda \cap E^{\prime \prime}=\delta C_{0}(\Gamma, \mathrm{Z})$. By Proposition 6.4, $D$ is in $K_{\phi}(\Gamma)$ if and only if it is contained in this fundamental domain. By Proposition 6.6, $D$ is in $K_{\phi-s t a b l e}(\Gamma)$ if and only if it is in the interior of this fundamental domain.

In the geometric invariant theory, we are particularly in good shape if semistable geometric objects are automatically stable. Let us now examine such cases in our context.

Definition. An element $\phi$ in $\partial C_{1}(\Gamma, \mathbf{R})$ is called nondegenerate if $\phi$-semistable Delony polyhedra are automatically $\phi$-stable, i.e.

$$
K_{\phi}(\Gamma)=K_{\phi-\text {-stable }}(\Gamma),
$$

or equivalently $K_{\phi}(\Gamma)=K_{\phi}^{0}(\Gamma)$.

Proposition 7.6. The following (1), (2), and (3) are equivalent, and imply $\left(2^{\prime}\right)$.

(1) $\phi$ is nondegenerate.

(2) $\operatorname{Sk}^{0}\left(K_{\phi-s t a b l e}(T)\right)=\operatorname{Sk}^{0}\left(K_{\phi}(T)\right)$.

(2) $\mathrm{Sk}^{0}\left(\mathrm{Del}_{\phi}\left(H^{1}(\Gamma, \mathbf{R})\right)\right)=H^{1}(\Gamma, \mathbf{Z})$.

(3) $\phi-\partial e(J) / 2$ is in the interior of a top-dimensional polyhedron in $\operatorname{Arr}(\Gamma)$.

Proof. (1) $\Rightarrow(2) \Rightarrow\left(2^{\prime}\right)$ is immediate.

$(2) \Rightarrow(1)$. Let $D$ be in $K_{\phi}(\Gamma)$, hence $D$ is in the fundamental domain $\partial^{-1}\left(\phi+\partial V_{J}(0)\right)$. But all the vertices of $D$ are in its interior by (2). Since the interior is convex, we see that $D$ is contained in the interior. Hence by Proposition 6.6, $D$ is in $K_{\phi-\text {-stable }}(\Gamma)$.

$(1) \Rightarrow(3)$. Let $\psi$ be the unique element in $E^{\prime \prime}$ with $\partial \psi=\phi$. Then as in the proof of Theorem 7.1, we see that

$$
P(\psi)=\bigcap_{T} \bigcap_{D} \pi^{\prime \prime}\left(D^{*}\right)
$$

with $\{I, T\}$ running over all the spanning forests of $\Gamma$ and $D$ running over all polyhedra satisfying $\operatorname{Supp}(D)=J-T$ and $D \in K_{\phi}(\Gamma)$, i.e. $\pi^{\prime \prime}\left(D^{*}\right) \ni \phi$. Such $D$ is uniquely determined by $T$ up to the translation by elements of $\Lambda \cap E^{\prime \prime}$ hence $\pi^{\prime \prime}\left(D^{*}\right)$ is uniquely determined by $T$. Moreover, the dimension of $\pi^{\prime \prime}\left(D^{*}\right)$ is equal to that of $E^{\prime \prime}$. Since $\psi$ is necessarily contained in the interior of $\pi^{\prime \prime}\left(D^{*}\right)$ for such $D$ by $(1)$, we see that $P(\psi)$ is a top dimensional polyhedron in $\operatorname{Par}\left(E^{\prime \prime}\right)$.

$(3) \Rightarrow(2)$. Let $\psi$ be as above. Then by Lemma 7.4, we have

$$
P(\psi)=\bigcap \pi^{\prime \prime}(V(\xi))
$$


with $\xi$ running over $\mathrm{Sk}^{0}\left(K_{\phi}(T)\right)$. Since $P(\psi)$ is a top-dimensional polyhedron and since $\psi$ is in its interior by (3), we see that $\psi$ is in the interior of all such $\pi^{\prime \prime}(V(\xi))$. Thus $\xi$ is in $\operatorname{Sk}^{0}\left(K_{\phi-s t a b l e}(\Gamma)\right)$. Q.E.D.

Definition. Let $\phi$ be in $\partial C_{1}(\Gamma, \mathbf{R})$. For $0 \leqslant r \leqslant h(\Gamma)=\operatorname{dim} H^{1}(\Gamma, \mathbf{R})$, we denote by $B_{r}=B_{r}(\Gamma ; \phi)$ the number of $\rho\left(H_{1}(\Gamma, \mathbf{Z})\right)$-equivalence classes of $r$-dimensional polyhedra in the Namikawa decomposition $\operatorname{Del}_{\phi}\left(H^{1}(\Gamma, \mathbf{R})\right)$, i.e. the number of $r$-cells in the $h(\Gamma)$-dimensional quotient cell complex

$$
\operatorname{Del}_{\phi}\left(H^{1}(\Gamma, \mathbf{R})\right) / \rho\left(H_{1}(\Gamma, \mathbf{Z})\right) \text {. }
$$

THEOREM 7.7. Let $\phi$ in $\partial C_{1}(\Gamma, \mathbf{R})$ be nondegenerate. Then

(1) there is a canonical bijection between the set of $\rho\left(H_{1}(\Gamma, Z)\right)$-equivalence classes of $h(\Gamma)$-dimensional polyhedra in $\operatorname{Del}_{\phi}\left(H^{1}(\Gamma, \mathbf{R})\right)$ and the set of spanning forests of $\Gamma$. In particular we have $B_{h(\Gamma)}(\Gamma ; \phi)=\operatorname{complexity}(\Gamma)$, and

(2) for $0 \leqslant r \leqslant h(\Gamma)$, we have $B_{r}(\Gamma ; \phi)=\left(\begin{array}{c}h(\Gamma) \\ r\end{array}\right) \cdot$ complexity $(\Gamma)$.

Proof. Since $\phi$ is nondegenerate, $\rho$ induces an isomorphism

$$
\rho: K_{\phi \text {-stable }}(\Gamma)=K_{\phi}(\Gamma) \rightarrow \operatorname{Del}_{\phi}\left(H^{1}(\Gamma, \mathbf{R})\right) .
$$

Consider the map

$$
K_{\phi}(\Gamma) \rightarrow\{\text { subsets of } J\}
$$

sending $D$ to $J^{\prime}=J-\operatorname{Supp}(D)$. Then by Proposition $6.6\left(1^{\prime}\right)$, the image consists exactly of those subsets $J^{\prime}$ of $J$ containing the set of edges of a spanning forest of $\Gamma$. Given such $J^{\prime}$, the set of $H_{1}(\Gamma, \mathbf{Z})$-equivalence classes of $D$ in $K_{\phi}(\Gamma)$ with $J^{\prime}=J-\operatorname{Supp}(D)$ is in one-to-one correspondence, via the map $D \mapsto b(D)$, with the intersection of $\partial e\left(J-J^{\prime}\right) / 2+\partial C_{1}(\Gamma, \mathbf{Z})$ with $\phi+$ rel.int $\partial V_{J}(0)$ by Proposition 5.1 and Proposition $6.6\left(1^{\prime}\right)$. The latter set is the interior of a fundamental domain of $\partial C_{1}(\Gamma, \mathbf{R})$ with respect to the lattice $\partial \delta_{J}, C_{0}(\Gamma, \mathbf{Z})$ by Corollary 6.3 applied to the graph $\left\{I, J^{\prime}\right\}$. Thus the number of $H_{1}(\Gamma, \mathrm{Z})$-equivalence classes of $D$ in $K_{\phi}(\Gamma)$ with $J^{\prime}=J-\operatorname{Supp}(D)$ is equal to the index $\left[\partial C_{1}(\Gamma, \mathbf{Z}): \partial \delta_{J}, C_{0}(\Gamma, \mathbf{Z})\right]$, which, by Kirchhoff-Trent's theorem in $\S 4$, is equal to the number of spanning forests in $\left\{I, J^{\prime}\right\}$. Applying this result to the case where $\left\{I, J^{\prime}\right\}$ is a spanning forest of $\Gamma$, we get (1). As for (2), we have seen so far that

$$
B_{r}=\sum_{J^{\prime}} \text { complexity }\left(\left\{I, J^{\prime}\right\}\right),
$$

where $J^{\prime}$ runs over all subsets of $J$ containing the set of edges of a spanning forest of $\Gamma$. Given a spanning forest $\{I, T\}$ of $\Gamma$, we know $|J-T|=h(\Gamma)$, hence there are $\left(\begin{array}{c}h(\mathrm{D}) \\ r\end{array}\right)$ different subsets $J^{\prime}$ of $J$ with $J^{\prime} \supset T$. Thus we conclude that

$$
B_{r}=\sum_{T}\left(\begin{array}{c}
h(\Gamma) \\
r
\end{array}\right)=\left(\begin{array}{c}
h(\Gamma) \\
r
\end{array}\right) \cdot \operatorname{complexity}(\Gamma)
$$


Remark. Proposition $7.6\left(2^{\prime}\right)$ is obviously equivalent to

$$
B_{0}(\Gamma ; \phi)=\text { complexity }(\Gamma)
$$

by Kirchhoff-Trent's theorem. This is a special case of Theorem 7.7 (2). It was pointed out by S. Usui that Proposition 7.6 (2') need not imply (1), (2) and (3).

REMARK. The 1-skeleton of the cell-complex dual to

$$
\operatorname{Del}_{\phi}\left(H^{1}(\Gamma, \mathbf{R})\right) / \rho\left(H_{1}(\Gamma, \mathbf{Z})\right)
$$

for a nondegenerate $\phi$, is the "spanning tree graph" of $\Gamma$ (cf. Harary [11]).

We have seen that $\phi$ is nondegenerate if and only if $\phi-\partial e(J) / 2$ belongs to the interior of a top-dimensional polyhedron in $\operatorname{Arr}(\Gamma)$.

Let us consider the other extreme. We know by Corollary 3.2 that

$$
\partial C_{1}(\Gamma, \mathbf{Z}) \subset \operatorname{Sk}^{0}(\operatorname{Arr}(\Gamma))
$$

They may not coincide, since $\left\{f_{I^{\prime}} ; I^{\prime}\right.$ elementary subsets of $\left.I\right\}$ in the dual of $\partial C_{1}(\Gamma, Z)$ need not be totally unimodular in general. We show this below in the case where $\Gamma$ is the simple complete graph with four vertices, i.e. the 1-skeleton of a tetrahedron ( $\$ 8$, Example (5)). This is obviously the simplest example of a graph without total unimodularity. Thus the coarsest decompositions among Namikawa decompositions need not be the ones we describe below in Proposition 7.8. Hence we have a negative answer to a question raised by Namikawa concerning the characterization of stable quasi-abelian varieties by minimality.

Proposition 7.8. For $\phi$ in $\partial C_{1}(\Gamma, \mathbf{R})$, the following are equivalent:

(1) $\phi-\partial e(J) / 2$ belongs to $\partial C_{1}(T, Z)$.

(2) There is a polyhedron $D$ in $K_{\phi}(\Gamma)$ with $\operatorname{Supp}(D)=J$.

In this case the following hold.

(3) $\operatorname{Del}_{\phi}\left(H^{1}(\Gamma, \mathbf{R})\right)$ is the translation by an element of $\rho(e(J) / 2)+H^{1}(\Gamma, \mathbf{Z})$ of the image under $\rho$ of the Voronoi decomposition $\operatorname{Vor}\left(H_{1}(\Gamma, \mathbf{R}), H_{1}(\Gamma, \mathbf{Z})\right.$ ).

(4) $B_{h(\Gamma)}(\Gamma ; \phi)=1$.

This is a re-interpretation of Corollary 6.2.

REMARK. (3) and (4) are properties about the Namikawa decomposition and are insufficient to characterize (1) and (2). For instance, let $\Gamma=\{I, J\}$ be such that $|I|=|J|=2$, with one edge joining the two vertices, while the other edge is a loop at one of the vertices. For any $\phi$ the Namikawa decomposition is identical, while $K_{\phi}(\Gamma)$ depends on whether $\phi-\partial e(J) / 2$ belongs to the interior of a one-dimensional polyhedron in $\operatorname{Arr}(\Gamma)$ or in a 0-dimensional polyhedron (cf. §8. Example (2i)).

8. Examples. In this section, we apply our theory in previous sections to various graphs $\Gamma=\{I, J\}$ and compute Namikawa decompositions for them.

(1) When $|I|=1$, i.e. the graph has only one vertex and all the edges are 
loops we have $\partial C_{1}(\Gamma, \mathbf{R})=0$ and $H_{1}(\Gamma, \mathbf{R})=H^{1}(\Gamma, \mathbf{R})=C_{1}(\Gamma, \mathbf{R})$. Thus automatically $\phi=0$ and $K_{\phi \text {-stable }}(\Gamma)=K_{\phi}(\Gamma)=K(\Gamma)=\operatorname{Del}_{\phi}\left(H^{1}(\Gamma, \mathbf{R})\right.$ ) (see Figure 3).

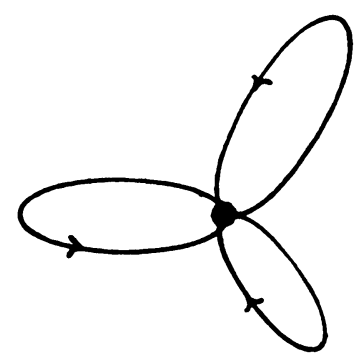

FIGURE 3

(2) When $|I|=2$ and $|J|=m$, the graph looks like Figure 4.

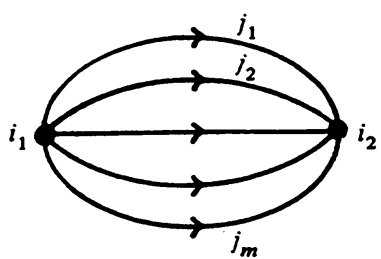

FIGURE 4

Then $\partial C_{1}(\Gamma, \mathbf{R})=\mathbf{R}\left(v_{1}-v_{2}\right) \subset C_{0}(\Gamma, \mathbf{R})=\mathbf{R} v_{1}+\mathbf{R} v_{2}, \partial \delta v_{1}=-\partial \delta v_{2}=$ $m\left(v_{1}-v_{2}\right)$ and $\partial e(J) / 2=m\left(v_{1}-v_{2}\right) / 2$. Since the elementary subsets of $I$ are $\left\{i_{1}\right\}$ and $\left\{i_{2}\right\}$, the polyhedral decomposition $\operatorname{Arr}(\Gamma)$ of $\partial C_{1}(\Gamma, \mathbf{R})$ is defined by $\left\{f_{1}, f_{2}=-f_{1}\right\}$, where $f_{1}(?)=\left[v_{1}\right.$, ? $]$ and $f_{2}(?)=\left[v_{2}\right.$, ?] are elements of $\partial C_{1}(\Gamma, \mathbf{Z})^{\swarrow}$. Arr $(\Gamma)$ looks like Figure 5.

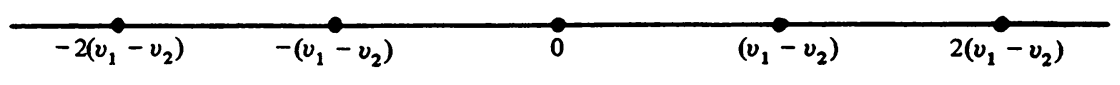

Figure 5

(2i) When $m=1$, the graph is a tree and looks like Figure 6.

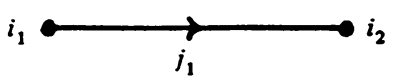

FIGURE 6

Hence $H_{1}(\Gamma, \mathbf{R})=H^{1}(\Gamma, \mathbf{R})=0$. In this case the Namikawa decomposition is obviously the same for any $\phi$, and consists of 0 . Let $\phi-\partial e(J) / 2=x\left(v_{1}-\right.$ $v_{2}$ ) with $x$ in $\mathbf{R}$. Then we have 


$$
\partial^{-1}\left(\phi+\partial V_{J}(0)\right)=\left\{y e_{1} ; x \leqslant y \leqslant x+1\right\} .
$$

By Propositions 6.4, 6.5 and 6.6, we have the following:

(2ia) When $x$ is not an integer, i.e. $x$ is nondegenerate, $K_{\phi-\text { stable }}(T)=K_{\phi}(\Gamma)$ consists of the 0 -dimensional polyhedron $D=([x]+1) e_{1}$, where $[x]$ is the Gauss symbol.

(2ib) On the other hand, if $x$ is an integer, then $K_{\phi \text {-stable }}(\Gamma)$ is empty and $K_{\phi}^{0}(\Gamma)$ consists of the 1-dimensional polyhedron

$$
D=\left\{y e_{1} ; x \leqslant y \leqslant x+1\right\} \text {. }
$$

$K_{\phi}(\Gamma)$ consists of $D$ and their faces $x e_{1}$ and $(x+1) e_{1}$.

(2ii) When $m=2$, the graph looks like Figure 7

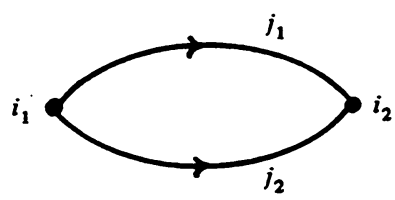

FIGURE 7

and $H_{1}(\Gamma, \mathbf{Z})=\mathbf{Z}\left(e_{1}-e_{2}\right)$. There are two spanning trees, $\left\{I,\left\{j_{1}\right\}\right\}$ and $\left\{I,\left\{j_{2}\right\}\right\}$. Let $\phi-\partial e(J) / 2=x\left(v_{1}-v_{2}\right)$ with $x$ in $\mathbf{R}$. Then we have

$$
\partial^{-1}\left(\phi+\partial V_{J}(0)\right)=\left\{y_{1} e_{1}+y_{2} e_{2} ; x \leqslant y_{1}+y_{2} \leqslant x+2\right\} \text {. }
$$

Thus again by Propositions 6.4, 6.5 and 6.6, we have the following:

(2iia) If $x=2 k$ is an even integer, then $K_{\phi}(\Gamma)$ consists of the translation by elements of $H_{1}(\Gamma, \mathbf{Z})=\mathbf{Z}\left(e_{1}-e_{2}\right)$ of

$$
D=\left\{y_{1} e_{1}+y_{2} e_{2} ; k \leqslant y_{1} \leqslant k+1, k \leqslant y_{2} \leqslant k+1\right\}
$$

and their faces. $K_{\phi}^{0}(\Gamma)$ consists of $H_{1}(\Gamma, Z)$-translates of $D$ and its vertex $(k+1) e_{1}+k e_{2}$, while $K_{\phi \text {-stable }}(\Gamma)$ consists of $H_{1}(\Gamma, \mathbf{Z})$-translates of $(k+1) e_{1}$ $+k e_{2}$.

(2iib) If $2 k<x<2 k+1$ for an integer $k$, then $\phi$ is nondegenerate and $K_{\phi \text {-stable }}(\Gamma)=K_{\phi}(\Gamma)$ consists of $H_{1}(\Gamma, \mathrm{Z})$-translates of

$$
\begin{aligned}
& D_{1}=\left\{y_{1} e_{1}+(k+1) e_{2} ; k \leqslant y_{1} \leqslant k+1\right\}, \\
& D_{2}=\left\{(k+1) e_{1}+y_{2} e_{2} ; k \leqslant y_{2} \leqslant k+1\right\},
\end{aligned}
$$

and their faces, $H_{1}(\Gamma, \mathrm{Z})$-translates of $(k+1) e_{1}+k e_{2}$ and $(k+1) e_{1}+(k+$ 1) $e_{2}$.

(2iic) If $x=2 k+1$ is an odd integer, then $K_{\phi}(\Gamma)$ consists of $H_{1}(\Gamma, \mathrm{Z})$ translates of

$$
D^{\prime}=\left\{y_{1} e_{1}+y_{2} e_{2} ; k+1 \leqslant y_{1} \leqslant k+2, k \leqslant y_{2} \leqslant k+1\right\}
$$

and their faces. $K_{\phi}^{0}(\Gamma)$ consists of $H_{1}(\Gamma, Z)$-translates of $D^{\prime}$ and its vertex 
$(k+2) e_{1}+k e_{2}$, while $K_{\phi-\text { stable }}(\Gamma)$ consists of $H_{1}(\Gamma, Z)$-translates of $(k+2) e_{1}$ $+k e_{2}$.

(2iid) Finally if $2 k+1<x<2 k+2$ for an integer $k$, $\phi$ is nondegenerate and $K_{\phi \text {-stable }}(\Gamma)=K_{\phi}(\Gamma)$ consists of $H_{1}(\Gamma, \mathrm{z})$-translates of

$$
\begin{aligned}
& D_{1}^{\prime}=\left\{y_{1} e_{1}+(k+1) e_{2} ; k+1 \leqslant y_{1} \leqslant k+2\right\}, \\
& D_{2}^{\prime}=\left\{(k+1) e_{1}+y_{2} e_{2} ; k+1 \leqslant y_{2} \leqslant k+2\right\}
\end{aligned}
$$

and their faces, $H_{1}(\Gamma, \mathrm{Z})$-translates of $(k+1) e_{1}+(k+1) e_{2}$ and $(k+1) e_{1}+$ $(k+2) e_{2}$.

Thus the Namikawa decompositions corresponding to these four cases look like Figure 8. For simplicity we denote $\rho\left(e_{1}\right)=-\rho\left(e_{2}\right)=\bar{e}_{1}$, which is a Z-base of $H^{1}(T, Z)$, while $\rho\left(H_{1}(T, Z)\right)$ is generated by $2 \bar{e}_{1}$. Note that $\operatorname{Supp}\left(D_{1}\right)=\operatorname{Supp}\left(D_{1}^{\prime}\right)=\left\{j_{1}\right\}$ and $\operatorname{Supp}\left(D_{2}\right)=\operatorname{Supp}\left(D_{2}^{\prime}\right)=\left\{j_{2}\right\}$.

(2 ii a)
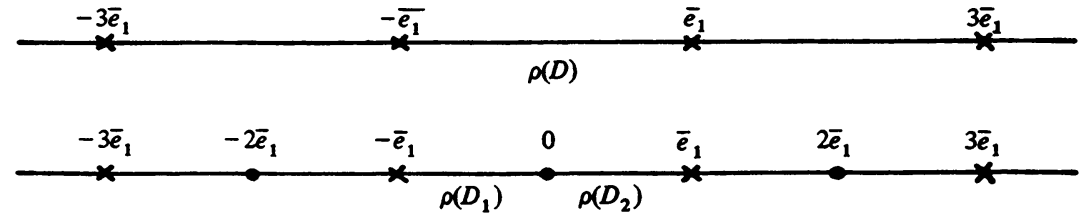

(2 ii c)

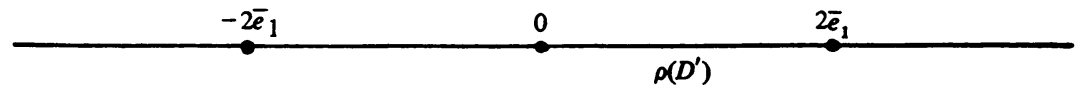

(2 ii d)

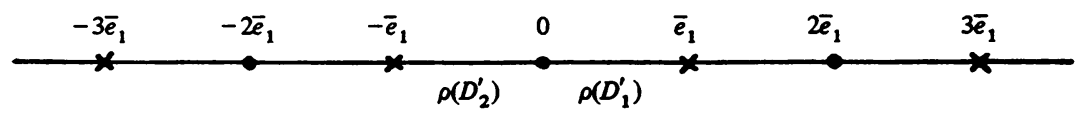

FIGURE 8

(2iii) When $m=3$, the graph looks like Figure 9 and $H_{1}(T, Z)=\mathbf{Z}\left(e_{1}-e_{2}\right)$ $\oplus \mathbf{Z}\left(e_{2}-e_{3}\right)$. There are three spanning trees, $\left\{I,\left\{j_{1}\right\}\right\},\left\{I,\left\{j_{2}\right\}\right\}$ and $\left\{I,\left\{j_{3}\right\}\right\}$. Let $\phi-\partial e(J) / 2=x\left(v_{1}-v_{2}\right)$ with $x$ in $\mathbf{R}$. Then

$$
\partial^{-1}\left(\phi+\partial V_{J}(0)\right)=\left\{y_{1} e_{1}+y_{2} e_{2}+y_{3} e_{3} ; x \leqslant y_{1}+y_{2}+y_{3} \leqslant x+3\right\} .
$$

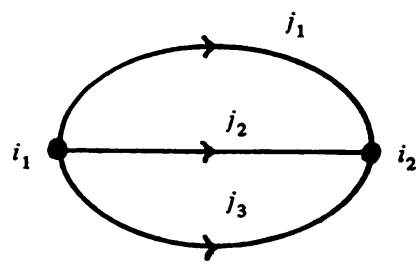

Figure 9

(2iiia) For instance, let us consider the case $\phi=0$, i.e. $x=-3 / 2$. This is a nondegenerate case. $K_{\phi-\text { stable }}(\Gamma)=K_{\phi}(\Gamma)$ consists of $H_{1}(\Gamma, \mathrm{Z})$-translates of 


$$
\begin{aligned}
& D_{12}=\left\{y_{1} e_{1}+y_{2} e_{2} ; 0 \leqslant y_{1} \leqslant 1,-1 \leqslant y_{2} \leqslant 0\right\}, \\
& D_{23}=\left\{y_{2} e_{2}+y_{3} e_{3} ; 0 \leqslant y_{2} \leqslant 1,-1 \leqslant y_{3} \leqslant 0\right\}, \\
& D_{13}=\left\{y_{1} e_{1}+y_{3} e_{3} ; 0 \leqslant y_{1} \leqslant 1,-1 \leqslant y_{3} \leqslant 0\right\}
\end{aligned}
$$

and their faces.

If we denote $\bar{e}_{1}=\rho\left(e_{1}\right)$ and $\bar{e}_{2}=\rho\left(e_{2}\right)$, then $\rho\left(e_{3}\right)=-\bar{e}_{1}-\bar{e}_{2}$, and the corresponding Namikawa decomposition looks like Figure 10 which is exactly the picture obtained by Deligne and Mumford in Mumford [22, p. 270].

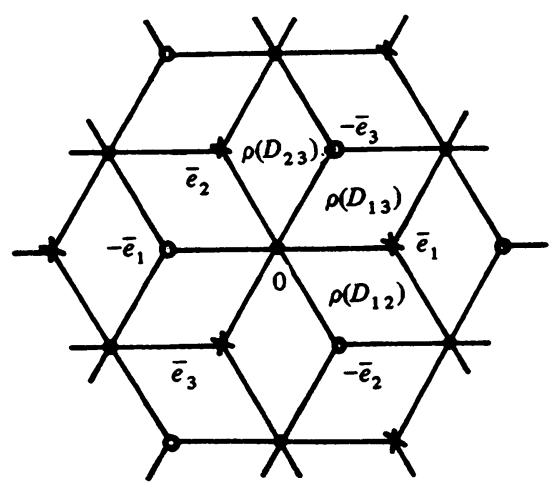

FIGURE 10

(2iiib) If $\phi=\left(v_{1}-v_{2}\right) / 2$ for instance, hence is degenerate, then we have

$$
\partial^{-1}\left(\phi+\partial V_{J}(0)\right)=\left\{y_{1} e_{1}+y_{2} e_{2}+y_{3} e_{3} ;-1 \leqslant y_{1}+y_{2}+y_{3} \leqslant 2\right\} .
$$

Thus the corresponding Namikawa decomposition $\operatorname{Del}_{\phi}\left(H^{1}(\Gamma, \mathbf{R})\right)$ of $H^{1}(\Gamma, \mathbf{R})$ consists of $\rho\left(H_{1}(\Gamma, \mathbf{Z})\right)$-translates of

$$
\rho\left(\left\{y_{1} e_{1}+y_{2} e_{2}+y_{3} e_{3}: 0 \leqslant y_{1} \leqslant 1,0 \leqslant y_{2} \leqslant 1,-1 \leqslant y_{3} \leqslant 0\right\}\right)
$$

and their faces and looks like Figure 11. Obviously the decomposition in (2iiia) is a subdivision of this.

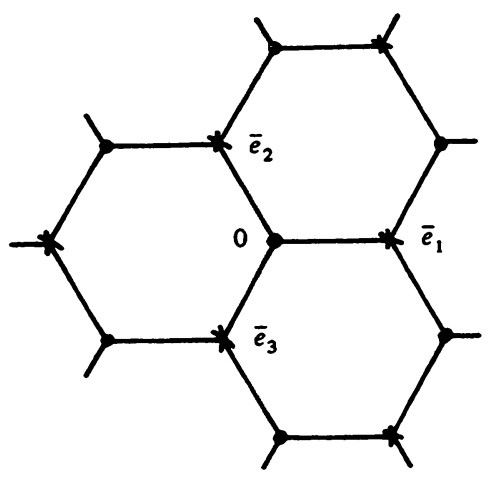

FIGURE 11 
(3) Consider the graph which looks like Figure 12 where there are $a, b$ and $c$ edges along the upper, middle and lower paths, respectively, with nonnegative integers $a, b$ and $c$. The cyclomatic number of this graph is 2 . Let $\bar{e}, \bar{e}^{\prime}$ and $\bar{e}^{\prime \prime}$ be the image under $\rho$ of one of the edges in the upper, middle and lower paths, respectively. We have $\bar{e}+\bar{e}^{\prime}+\bar{e}^{\prime \prime}=0$ and $H^{1}(\Gamma, \mathbf{Z})=\mathbf{Z} \bar{e} \oplus$ $\mathbf{Z} \bar{e}^{\prime}$, and $\rho\left(H_{1}(\Gamma, \mathbf{Z})\right)$ has a $\mathbf{Z}$-basis consisting of $(a+c) \bar{e}+c \bar{e}^{\prime}$ and $c \bar{e}+(b$ $+c) \bar{e}^{\prime}$. The number of spanning trees is

$$
a b+b c+c a=\operatorname{det}\left|\begin{array}{cc}
a+c & c \\
c & b+c
\end{array}\right| .
$$

They are obtained by deleting one edge each from two of the three paths.

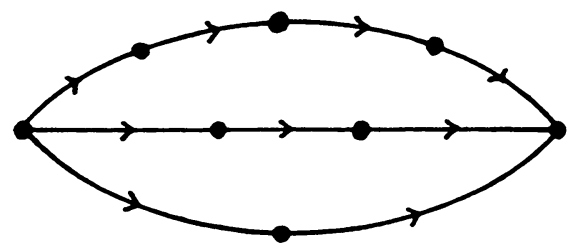

FIGURE 12

Consider tiles in $H^{1}(\Gamma, \mathbf{R})$ of the following shapes and colors: $a b$ colors of the tile of the shape

$$
\left\{y \bar{e}+y^{\prime} \bar{e}^{\prime} ; 0 \leqslant y \leqslant 1,0 \leqslant y^{\prime} \leqslant 1\right\},
$$

ac colors of the tile of the shape

$$
\left\{y \bar{e}+y^{\prime \prime} \bar{e}^{\prime \prime} ; 0 \leqslant y \leqslant 1,0 \leqslant y^{\prime \prime} \leqslant 1\right\}
$$

and $b c$ colors of the tile of the shape

$$
\left\{y^{\prime} \bar{e}^{\prime}+y^{\prime \prime} \bar{e}^{\prime \prime} ; 0 \leqslant y^{\prime} \leqslant 1,0 \leqslant y^{\prime \prime} \leqslant 1\right\} .
$$

For a nondegenerate $\phi$, the corresponding Namikawa decomposition $\operatorname{Del}_{\phi}\left(H^{1}(\Gamma, \mathbf{R})\right)$ is a colored tiling, with these tiles, of the plane $H^{1}(\Gamma, \mathbf{R})$, invariant under the translation by $\rho\left(H_{1}(\Gamma, \mathrm{Z})\right)$. The coloring corresponds to the labeling of the 2-dimensional polyhedra by spanning trees (cf. Theorem 7.7).

For simplicity, let us ignore the colors of the tiles. Even then, there are, in general, many different ways of tiling.

(i) When $b=c=1$, for instance, the tiling is always obtained by $\rho\left(H_{1}(\Gamma, \mathbf{Z})\right)$-translating the block of tiles of the shape in Figure 13.

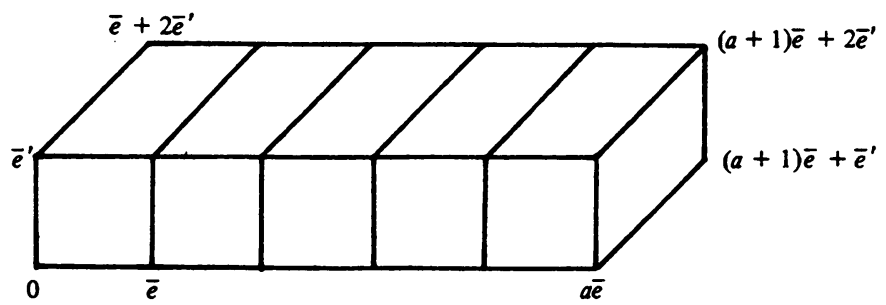

FIGURE 13 
(ii) When $a=b=2$ and $c=1$, there are exactly two completely different ways of tiling, the ones obtained by $\rho\left(H_{1}(\Gamma, Z)\right)$-translating the block of the shapes in Figure 14. We in fact get both kinds for suitable choices of nondegenerate $\phi$.

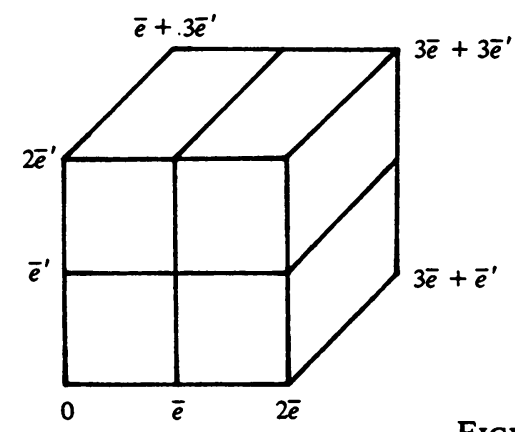

FigURE 14

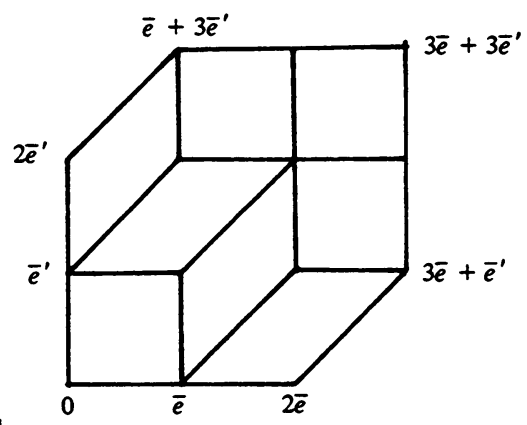

(iii) When $a=b=c=2$, we can show that there are exactly five different ways of tiling ignoring the colors.

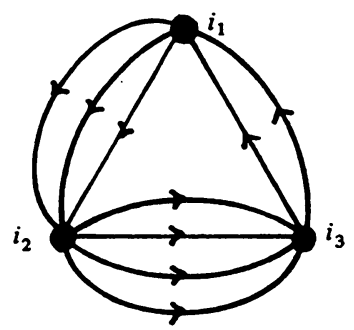

FIGURE 15

(4) When $|I|=3$, the graph looks like Figure 15 where there are $a, b$ and $c$ edges opposite to the vertices $i_{1}, i_{2}$ and $i_{3}$, respectively. Hence $\partial C_{1}(T, Z)=$ $\mathbf{Z}\left(v_{1}-v_{2}\right) \oplus \mathbf{Z}\left(v_{2}-v_{3}\right)$. The elementary subsets of $I$ are all the subsets of cardinality one or two. Thus if $f_{1}(?)=\left[v_{1}\right.$, ?] and $f_{3}(?)=\left[v_{3}, ?\right]$, then $f_{1}$ and $f_{3}$ form the base of $\partial C_{1}(\Gamma, Z)$ dual to $\left\{v_{1}-v_{2},-v_{2}+v_{3}\right\}$, and $\left\{ \pm f_{1}, \pm f_{3}, \pm\left(f_{1}+f_{3}\right)\right\}$ defines the polyhedral decomposition $\operatorname{Arr}(\Gamma)$ of the plane $\partial C_{1}(\Gamma, \mathbf{R})$, which looks like Figure 16.

(5) Finally let us consider the graph in Figure 17 which is the 1-skeleton of a tetrahedron. Its cyclomatic number is three, and $H_{1}(\Gamma, \mathbf{Z})$ is generated over $\mathrm{Z}$ by $-e_{1}+e_{3}^{\prime}+e_{2},-e_{2}+e_{1}^{\prime}+e_{3}$ and $-e_{3}+e_{2}^{\prime}+e_{1}$. The number of spanning trees is

$$
16=\operatorname{det}\left|\begin{array}{rrr}
3 & -1 & -1 \\
-1 & 3 & -1 \\
-1 & -1 & 3
\end{array}\right|
$$




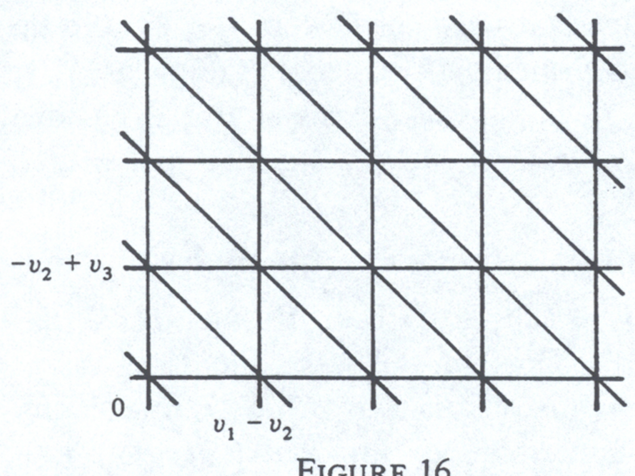

FIGURE 16

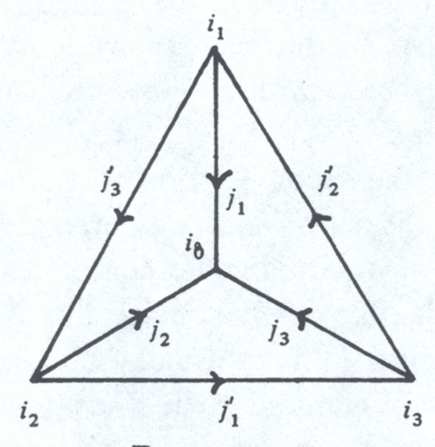

FIGURE 17

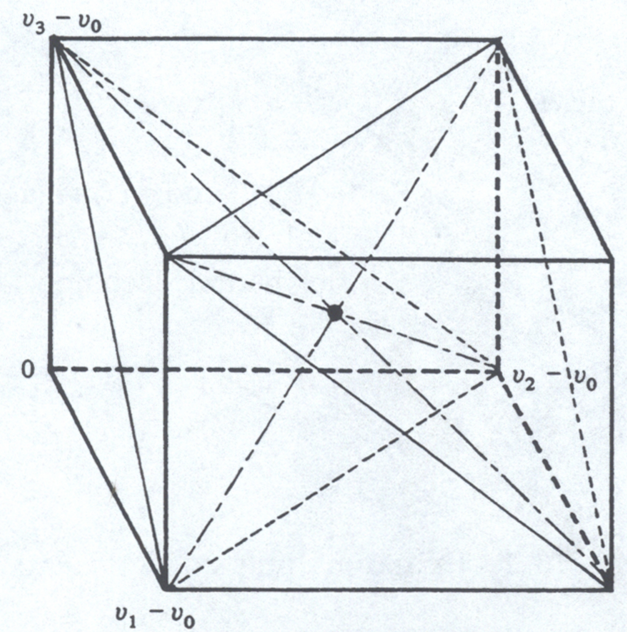

FIGURE 18 
There are 14 elementary subsets $I^{\prime}$ of $I=\{0,1,2,3\}$, and they are subsets of $I$ of one, two or three elements. If we denote $f_{1}(?)=\left[v_{1}, ?\right], f_{2}(?)=\left[v_{2}, ?\right]$ and $f_{3}(?)=\left[v_{3}, ?\right]$, then $\left\{f_{1}, f_{2}, f_{3}\right\}$ is a Z-basis of $\partial C_{1}(\Gamma, \mathbf{Z})^{-}$dual to the Z-basis $\left\{v_{1}-v_{0}, v_{2}-v_{0}, v_{3}-v_{0}\right\}$ of $\partial C_{1}(\Gamma, Z)$. The polyhedral decomposition $\operatorname{Arr}(\Gamma)$ is defined by

$$
\left\{ \pm f_{1}, \pm f_{2}, \pm f_{3}, \pm\left(f_{1}+f_{2}\right), \pm\left(f_{2}+f_{3}\right), \pm\left(f_{3}+f_{1}\right), \pm\left(f_{1}+f_{2}+f_{3}\right)\right\} \text {. }
$$

Note that $\left\{f_{1}+f_{2}, f_{2}+f_{3}, f_{3}+f_{1}\right\}$ is an $\mathbf{R}$-basis of $\partial C_{1}(T, \mathbf{R})^{-}$but is not a $\mathbf{Z}$-basis of $\partial \mathbf{C}_{1}(\Gamma, \mathbf{Z})^{-}$. Thus in this case the total unimodularity is not satisfied. Indeed, $\operatorname{Arr}(\Gamma)$ is obtained by $\partial C_{1}(\Gamma, \mathrm{Z})$-translating the subdivision in Figure 18 of the standard cube. Note that $\left\{\left(v_{1}-v_{0}\right)+\left(v_{2}-v_{0}\right)+\left(v_{3}-\right.\right.$ $\left.\left.v_{0}\right)\right\} / 2$ is a vertex of $\operatorname{Arr}(\Gamma)$, but it does not belong to $\partial C_{1}(\Gamma, \mathbf{Z})$ (cf. the paragraph before Proposition 7.8).

\section{Chapter II. CURVES OVER AN ALGEBRAICALIy CLOSED FIELD}

9. The graph of a curve. In this chapter, we let $X$ be a reduced, connected and complete algebraic curve with at most ordinary double points over an algebraically closed field $k$.

Let $X=\cup_{i \in I} X_{i}$ be the decomposition of $X$ into irreducible components. We denote by $\left\{Q_{j}\right\}_{j \in J}$ the set of double points of $X$. Then $Q_{j}$ is either (i) an ordinary double point of an irreducible component $X_{i}$, or (ii) a transversal intersection of two irreducible components $X_{i}$ and $X_{i^{\prime}}$.

DefinItion. We associate to $X$, as usual, a connected graph $\Gamma(X)=\{I, J\}$ with $I$ as the set of vertices and with $J$ as the set of edges. (i) $j$ in $J$ corresponds to a loop at the vertex $i$, if $Q_{j}$ is an ordinary double point of an irreducible component $X_{i}$. (ii) $j$ in $J$ is an edge joining vertices $i$ and $i^{\prime}$ if $Q_{j}$ is a transversal intersection of irreducible components $X_{i}$ and $X_{i^{\prime}}$ (see Figure 19).
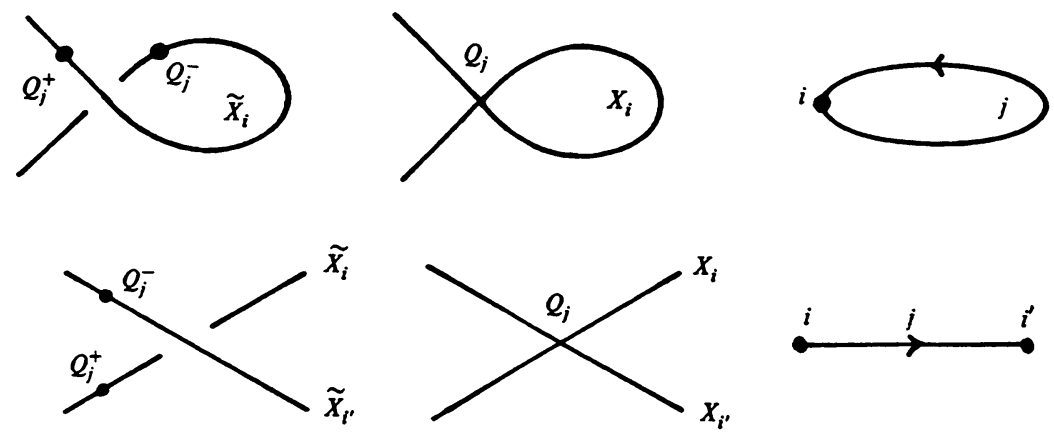

FIGURE 19

Every graph even with loops and multiple edges appears as the graph of a curve in this way.

As in Chapter I, we assign and fix an arbitrary orientation to $\Gamma(x)$. 

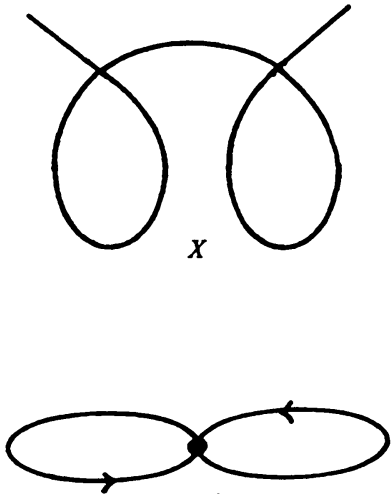

$\Gamma(X)$

FIGURE 20
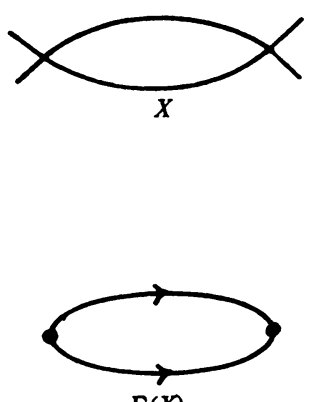

$\Gamma(X)$

FIGURE 21
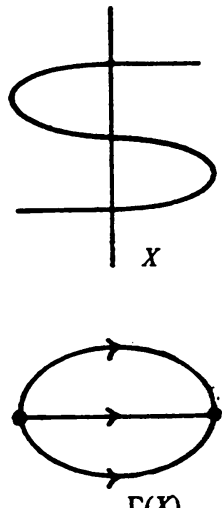

$\Gamma(X)$

FIGURE 22

Corresponding to the orientation, we name two points on the normalization

$$
\tilde{X}=\coprod_{i \in I} \tilde{X}_{i}
$$

of $X$ lying above $Q_{j}$ as $Q_{j}^{+}$and $Q_{j}^{-}$, where (i) $Q_{j}^{+}$and $Q_{j}^{-}$are on the normalization $\tilde{X}_{i}$ of $X_{i}$, if $j$ is a loop at $i$, while (ii) $Q_{j}^{+}$is on $\tilde{X}_{i}$ and $Q_{j}^{-}$is on $\tilde{X}_{i^{\prime}}$, if $j$ is an edge from $i$ to $i^{\prime}$.

DefinItION. For a subset $J^{\prime}$ of $J$, let

$$
\sigma\left(J^{\prime}\right): X\left(J^{\prime}\right) \rightarrow X
$$

be the partial normalization obtained by the blowing up along $\left\{Q_{j}\right\}_{j \in J-J^{\prime}}$. When $J^{\prime}=\varnothing, X(\varnothing)$ is the normalization of $X$, which we also denote by $\tilde{X}=\amalg \tilde{X}_{i}$, where $\tilde{X}_{i}$ is the normalization of the irreducible component $X_{i}$.

Obviously the graph $\Gamma\left(X\left(J^{\prime}\right)\right)$ is the spanning subgraph $\left\{I, J^{\prime}\right\}$. Note that $X(J)=X$.

We can apply the results of Chapter I to the connected graph $\Gamma(X)$.

EXAMPLE. (1) $X$ is an irreducible curve with two nodes. In this case $\Gamma(X)$ is a special case of $\S 8,(1)$ (Figure 20).

(2) $X$ has two nonsingular components meeting at two points. In this case $\Gamma(X)$ is the one we dealt with in $\$ 8$, (2ii) (Figure 21).

(3) $X$ has two nonsingular components meeting at three points, i.e. $X$ looks like a "dollar sign". The graph $\Gamma(X)$ is the one we dealt with in $\S 8$, (2iii) (Figure 22).

10. Line bundles. In this section we study torsion-free, everywhere rank one $\theta_{x}$-modules on a curve $X$. We eventually compactify the generalized Jacobian variety of $X$ by adding points corresponding to those which are not locally free. 
DefINITION. By a line bundle $F$ on a curve $X$, we mean a torsion-free (= depth one) everywhere rank one $\theta_{X}$-module. In particular the length of the stalk $F_{x_{i}}$ over $\theta_{X, x_{i}}$ is one for each generic point $x_{i}$ of $X$. We denote by $\mathrm{LB}(X)$ the set of isomorphism classes of line bundles on $X$.

Locally free line bundles form the Picard group $\operatorname{Pic}(X)$ of $X$. Obviously we have $\operatorname{Pic}(X) \subset \mathrm{LB}(X)$ and, moreover, $\operatorname{Pic}(X)$ acts on $\operatorname{LB}(X)$ by $F \mapsto L \otimes_{\theta_{X}} F$ for $F$ in $\operatorname{LB}(X)$ and $L$ in $\operatorname{Pic}(X)$.

It is well known that for $F$ in $\operatorname{LB}(X)$, the $\theta_{X, x}$-module $F_{x}$ is isomorphic to $\Theta_{X, x}$ at a smooth point $x$ of $X$, and for $j$ in $J$, the $\Theta_{X, Q_{j}}$-module $F_{Q_{j}}$ is either isomorphic to $\Theta_{X, Q_{j}}$ or to the maximal ideal $\mathfrak{N}_{X, Q_{j}}$, which in turn is isomorphic to the direct image of the normalization $\Theta_{\tilde{X}, Q_{j}}$.

Definition. For $F$ in $\operatorname{LB}(X)$, we denote

$$
J(F)=\left\{j \in J ; F \text { is locally free at } Q_{j}\right\} .
$$

In particular, we have $J(F)=J$, if $F$ is in $\operatorname{Pic}(X)$.

CONVEnTION. We always identify modules on partial normalizations $X\left(J^{\prime}\right)$ for $J^{\prime} \subset J$ with their direct images onto $X$ by $\sigma\left(J^{\prime}\right): X\left(J^{\prime}\right) \rightarrow X$.

From what we remarked above, we obviously get the following:

Proposition 10.1. For a subset $J^{\prime}$ of $J$, the set $\left\{F \in \operatorname{LB}(X) ; J(F)=J^{\prime}\right\}$ can be identified with the set $\operatorname{Pic}\left(X\left(J^{\prime}\right)\right)$ of (direct images onto $\left.X\right)$ of locally free line bundles on $X\left(J^{\prime}\right)$. In particular we have

$$
\mathrm{LB}(X)=\coprod_{J^{\prime} \subset J} \operatorname{Pic}\left(X\left(J^{\prime}\right)\right) \text {. }
$$

The pull-back

$$
\sigma^{*} F=\theta_{\tilde{X}} \otimes_{\theta_{x}} F
$$

of $F$ in $\operatorname{LB}(X)$ by $\sigma: \tilde{X} \rightarrow X$ is a coherent sheaf on $\tilde{X}$ of rank one on each component, hence $\sigma^{*} F$ modulo its $\theta_{\tilde{x}}$-torsion is a line bundle on $\tilde{X}$. If $F$ belongs to $\operatorname{Pic}\left(X\left(J^{\prime}\right)\right)$ as in Proposition 10.1, then $\sigma^{*} F / \theta_{\tilde{x}}$-torsion coincides with the pull-back of $F$ by $\tilde{X} \rightarrow X\left(J^{\prime}\right)$. Since

$$
\operatorname{LB}(\tilde{X})=\operatorname{Pic}(\tilde{X})=\prod_{i \in I} \operatorname{Pic}\left(\tilde{X}_{i}\right)
$$

a line bundle $L$ on $\tilde{X}$ can be identified with a set $\left(L_{i}\right)_{i \in I}$ of line bundles, one on each component $\tilde{X}_{i}$. Thus we have the following:

DEFINITION. We denote by

$$
\mathcal{E}: \operatorname{LB}(X) \rightarrow \operatorname{Pic}(\tilde{X})
$$

the map defined by

$$
\mathcal{L}(F)=\left(\mathcal{E}_{i}(F)\right)_{i \in I}=\Theta_{\tilde{X}} \otimes_{\theta_{x}} F / \Theta_{\tilde{x}} \text {-torsion }
$$

for $F$ in $\operatorname{LB}(X)$. 
We are now ready to compute $\operatorname{LB}(X)$.

Proposition 10.2. For a subset $J^{\prime}$ of $J$, we have an exact sequence of abelian groups

$$
0 \rightarrow H^{1}\left(\left\{I, J^{\prime}\right\}, k^{*}\right) \rightarrow \operatorname{Pic}\left(X\left(J^{\prime}\right)\right) \stackrel{\mathfrak{e}}{\rightarrow} \operatorname{Pic}(\tilde{X}) \rightarrow 0 .
$$

In particular, we have

$$
0 \rightarrow H^{1}\left(\Gamma(X), k^{*}\right) \rightarrow \operatorname{Pic}(X) \stackrel{\mathfrak{L}}{\rightarrow} \operatorname{Pic}(\tilde{X}) \rightarrow 0 .
$$

COROLLARY 10.3. The arithmetic genus of a connected curve $X$ is given by

$$
\sum_{i \in I} \operatorname{genus}\left(\tilde{X}_{i}\right)+h(\Gamma(X))
$$

where $h(\Gamma(X))=|J|-|I|+1$ is the cyclomatic number of the graph $\Gamma(X)$ defined before Proposition 4.1.

REMARK. When $k=\mathrm{C}$ is the field of complex numbers, we get similarly an exact sequence

$$
0 \rightarrow H^{1}(\Gamma(X), \mathbf{Z}) \rightarrow H^{1}(X, \mathbf{Z}) \rightarrow H^{1}(\tilde{X}, \mathbf{Z}) \rightarrow 0 .
$$

Proof of Proposition 10.2. Replacing $J^{\prime}$ by $J$, we may assume without loss of generality that $J^{\prime}=J$. We have an exact sequence of abelian sheaves on $X$

$$
1 \rightarrow \theta_{X}^{*} \rightarrow \theta_{\bar{X}}^{*} \stackrel{\alpha}{\rightarrow} \prod_{j \in J} k\left(Q_{j}\right)^{*} \rightarrow 1
$$

where $k\left(Q_{j}\right)^{*}$ is the sheaf whose stalk at $Q_{j}$ is the set of nonzero elements of the residue field of $Q_{j}$ and 1 at other points. $\theta_{\tilde{X}}^{*}=\Pi_{i \in I} \theta_{\tilde{X}_{i}}^{*}$ and $\alpha$ is the map defined as follows: (i) At smooth points of $X$, the homomorphism $\alpha$ sends every element of the stalk to 1 , (ii) at $Q_{j}$ with $j \in J$ a loop at $i \in I, \alpha$ sends an element $u$ of the stalk $\theta_{\tilde{X}, Q_{j}}=\left(\theta_{\tilde{X}_{i}, Q_{j}} \cap \theta_{\tilde{x}_{i}, Q_{j}^{-}}\right)^{*}$ to $u\left(Q_{j}^{+}\right) / u\left(Q_{j}^{-}\right)$, and (iii) at $Q_{j}$ with $j \in J$ an edge from $i$ to $i^{\prime}, \alpha$ sends an element $u=\left(u^{+}, u^{-}\right)$of the stalk $\theta_{\bar{X}, Q_{j}}^{*}=\theta_{\dot{X}_{i}, Q_{j}^{+}}^{*} \times \theta_{\bar{X}_{i}, Q_{j}^{-}}^{*}$ to $u^{+}\left(Q_{j}^{+}\right) / u^{-}\left(Q_{j}^{-}\right)$. Here $Q_{j}^{+}$and $Q_{j}^{-}$denote the points of $\tilde{X}$ lying above $Q_{j}$ as in the beginning of this section, and $u\left(Q_{j}^{+}\right), u^{+}\left(Q_{j}^{+}\right)$etc. denote the evaluation of the sections $u, u^{+}$etc. at those points, i.e. the image in the residue field. We thus have an exact sequence

$$
1 \rightarrow H^{0}\left(\theta_{X}^{*}\right) \rightarrow H^{0}\left(\theta_{\tilde{X}}^{*}\right) \rightarrow \prod_{j \in J} k^{*} \rightarrow \operatorname{Pic}(X) \rightarrow \operatorname{Pic}(\tilde{X}) \rightarrow 1 .
$$

It is immediate to see that the map $H^{0}\left(\theta_{\tilde{X}}^{*}\right) \stackrel{\alpha}{\rightarrow} \Pi_{j \in J} k^{*}$ coincides with the coboundary map $\delta: C_{0}\left(\Gamma(X), k^{*}\right) \rightarrow C_{1}\left(\Gamma(X), k^{*}\right)$.

REMARK. By the definition of $J(F)$ and $\mathcal{L}(F)$ for a line bundle $F \in \mathrm{LB}(X)$, we have an exact sequence of $\theta_{x}$-modules

$$
0 \rightarrow F \rightarrow \mathcal{L}(F) \rightarrow \bigoplus_{j \in J(F)} k\left(Q_{j}\right) \rightarrow 0
$$


where $k\left(Q_{j}\right)$ is the sheaf on $X$ concentrated at $Q_{j}$ with the residue field as the stalk there. We analyze how the surjection $\mathcal{L}(F) \rightarrow \bigoplus_{j \in J(F)} k\left(Q_{j}\right)$ depends on a cochain in $C_{1}\left(\{I, J(F)\}, k^{*}\right)$ later in $\$ 12$ where we introduce the notion of presentation.

DEFINITION. We define a surjective homomorphism

$$
\text { deg: } \operatorname{Pic}(\tilde{X}) \rightarrow C_{0}(\Gamma(X), Z)
$$

by $\operatorname{deg} L=\sum_{i \in I} \operatorname{deg}_{\tilde{X}_{i}}\left(L_{i}\right) v_{i}$, where $L=\left(L_{i}\right)_{i \in I}$ is $\operatorname{in} \operatorname{Pic}(\tilde{X})=\Pi_{i \in I} \operatorname{Pic}\left(\tilde{X}_{i}\right)$ and $\operatorname{deg}_{\tilde{X}_{i}}\left(L_{i}\right)$ is the degree of the invertible sheaf $L_{i}$ on $\tilde{X}_{i}$.

Proposmion 10.4. For a line bundle $F$ in $\mathrm{LB}(X)$, its Euler-Poincaré characteristic is given by

$$
\chi(F)=[v(I), \operatorname{deg} \mathcal{L}(F)+d(J-J(F)) / 2]+\chi\left(\theta_{x}\right),
$$

where $d\left(J^{\prime}\right)$ is the graph-theoretical degree defined immediately after Proposition 4.1.

Proof. From the exact sequences

$$
\begin{aligned}
& 0 \rightarrow F \rightarrow \mathscr{L}(F) \rightarrow \bigoplus_{j \in J(F)} k\left(Q_{j}\right) \rightarrow 0 \text { and } \\
& 0 \rightarrow \theta_{X} \rightarrow \theta_{\bar{X}} \rightarrow \bigoplus_{j \in J} k\left(Q_{j}\right) \rightarrow 0
\end{aligned}
$$

we get $\chi(F)=\chi(\mathscr{L}(F))-|J(F)|$ and $\chi\left(\theta_{X}\right)=\chi\left(\theta_{\tilde{X}}\right)-|J|=\sum_{i \in I} \chi\left(\theta_{\tilde{X}_{i}}\right)-$ $|J|$. But by the Riemann-Roch theorem, we see that

$$
\chi(\mathcal{L}(F))=\sum_{i \in I} \chi\left(\mathscr{L}_{i}(F)\right)=\sum_{i \in I}\left(\operatorname{deg}_{\tilde{x}_{i}}\left(\mathscr{E}_{i}(F)\right)+\chi\left(\Theta_{\tilde{X}_{i}}\right)\right) .
$$

Hence we conclude $\chi(F)-\chi\left(\theta_{X}\right)=[v(I), \operatorname{deg} \mathcal{L}(F)]+|J-J(F)|$, which is equal to $[v(I), \operatorname{deg} \mathcal{L}(F)+d(J-J(F)) / 2]$ by Lemma 4.2.

Definimion. We denote by $L B^{0}(X)$ the subset of $\operatorname{LB}(X)$ consisting of line bundles $F$ with $\chi(F)=\chi\left(\theta_{X}\right)$, i.e.

$$
[v(I), \operatorname{deg} \mathcal{L}(F)+d(J-J(F)) / 2]=0 .
$$

Obviously $\mathrm{LB}^{0}(X)$ contains the subgroups $\mathrm{LB}^{0}(X) \cap \operatorname{Pic}(X) \supset \operatorname{Pic}^{0}(X)$ of $\operatorname{Pic}(X)$ consisting of locally free line bundles $F$ of total degree $[v(I)$, $\operatorname{deg} \mathcal{L}(F)]=0$ and $\operatorname{deg} \mathcal{L}(F)=0$, respectively. $\operatorname{Pic}^{0}(X)$ acts on $\mathrm{LB}^{0}(X)$ by tensor product $F \mapsto L \otimes_{\theta_{X}} F$ for $F$ in $\operatorname{LB}^{0}(X)$ and $L$ in $\operatorname{Pic}^{0}(X)$.

We now consider the set $K(\Gamma(X))$ of Delony polyhedra for the graph $\Gamma(X)$ we introduced in $\$ 5$. Its 0-skeleton is

$$
\mathrm{Sk}^{0}\left(K(\Gamma(X))=C_{1}(\mathrm{~F}(X), \mathrm{Z}),\right.
$$

which acts on $K(\Gamma(X))$ by translation. We consider the action of the subgroup $H_{1}(\Gamma(X), \mathrm{Z})$. 
DEFINITION. We denote

$$
\bar{K}(\Gamma(X))=K(\Gamma(X)) / H_{1}(\Gamma(X), \mathbf{Z}),
$$

the set of equivalence classes of Delony polyhedra for $\Gamma(X)$ with respect to the translation action of $H_{1}(\Gamma(X), Z)$.

It is easy to see that the face relation in $\bar{K}(\Gamma(X))$ can be induced from that of $K(\Gamma(X))$, i.e. for $\bar{D}^{\prime}$ and $\bar{D}$ in $\bar{K}(\Gamma(X)), \bar{D}^{\prime}$ is a face of $\bar{D}$ if there exist $D^{\prime}$ in $\bar{D}^{\prime}$ and $D$ in $\bar{D}$ such that $D^{\prime}$ is a face of $D$. Thus $\bar{K}(\Gamma(X))$ is a complex, whose 0-skeleton is

$$
\operatorname{Sk}^{0}(\bar{K}(\Gamma(X)))=C_{1}(\Gamma(X), \mathbf{Z}) / H_{1}(\Gamma(X), \mathbf{Z}) \stackrel{\partial}{\rightarrow} \partial C_{1}(\Gamma(X), \mathbf{Z}) .
$$

$\operatorname{dim} \bar{D}$ and Supp $\bar{D}$ can be well defined as $\operatorname{dim} D$ and Supp $D$, respectively, for a $D$ in $\bar{D}$. Although $b(D)$ depends on a particular choice of $D$ in $\bar{D}$, we see that $\partial b(D)$ is uniquely determined, which we denote by $\partial b(\bar{D})$. Note also that the subset $\partial \bar{D}$ of $\partial C_{1}(\Gamma(X), \mathbf{R})$ makes sense.

The motivation for the introduction of this quotient complex is the following:

THEOREM 10.5. There exists a canonical surjective map

$$
\bar{D}: \mathrm{LB}^{0}(X) \rightarrow \bar{K}(\Gamma(X))
$$

which

(1) induces a bijection from the set of $\operatorname{Pic}^{0}(X)$-orbits in $\mathrm{LB}^{0}(X)$ onto $\bar{K}(\Gamma(X))$, i.e.

$$
\bar{D}: \mathrm{LB}^{0}(X) / \operatorname{Pic}^{0}(X) \stackrel{\sim}{\rightarrow} \bar{K}(\Gamma(X)),
$$

(2) is compatible with the order, i.e. for $F$ and $F^{\prime}$ in $\operatorname{LB}^{0}(X), F$ is in the "closure" of the $\operatorname{Pic}^{0}(X)$-orbit of $F^{\prime}$ if and only if $\bar{D}\left(F^{\prime}\right)$ is a face of $\bar{D}(F)$, satisfies

(3) Supp $\bar{D}(F)=J-J(F)$,

(4) $\operatorname{deg} \mathcal{E}(F)+d(J-J(F)) / 2=\partial b(\bar{D}(F))$, for all $F$ in $\mathrm{LB}^{0}(X)$, and

(5) fits into the following commutative diagram:

$$
\begin{aligned}
& \operatorname{LB}^{0}(X) \stackrel{\bar{D}}{\longrightarrow} \bar{K}(\Gamma(X))
\end{aligned}
$$

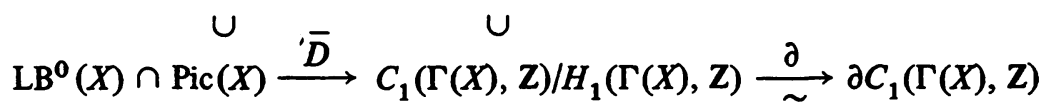

$$
\begin{aligned}
& \text { ก } \\
& \operatorname{Pic}(X) \stackrel{\mathfrak{E}}{\longrightarrow} \operatorname{Pic}(\tilde{X}) \longrightarrow C_{0}(\Gamma(X), Z) .
\end{aligned}
$$


Proof. If $F$ is in $\operatorname{LB}^{0}(X)$, we have

$$
[v(I), \operatorname{deg} \mathcal{L}(F)+d(J-J(F)) / 2]=0
$$

by Proposition 10.4. For simplicity let us denote $J^{\prime \prime}=J-J(F)$. Since $\Gamma(X)$ is connected, deg $\mathcal{E}(F)+d\left(J^{\prime \prime}\right) / 2$ is necessarily contained in $\partial C_{1}(\Gamma(X), \mathbf{Z})$ by what we remarked at the beginning of \$4. Consider the expression $d\left(J^{\prime \prime}\right) / 2-\partial e\left(J^{\prime \prime}\right) / 2$. By definition, this is equal to $\sum_{i \in I} a_{i} v_{i} / 2$, where

$a_{i}=\mid\left\{j \in J^{\prime \prime}\right.$; one end of $j$ is at $i$ with loops at $i$ counted twice $\} \mid$

$$
\begin{aligned}
& -\sum_{j \in J^{\prime \prime}}\left[v_{i}, \partial e_{j}\right] \\
= & 2 \mid\left\{j \in J^{\prime \prime} ; j \text { loop at } i\right\} \mid+\sum_{j \in J^{\prime \prime}}\left(\left[v_{i}, \partial e_{j}\right]^{2}-\left[v_{i}, \partial e_{j}\right]\right),
\end{aligned}
$$

which is an even integer, since $\left[v_{i}, \partial e_{j}\right]=0$ or \pm 1 . Thus $d\left(J^{\prime \prime}\right) / 2-\partial e\left(J^{\prime \prime}\right) / 2$ is contained in $C_{0}(T(X), Z)$. Hence $\operatorname{deg} \mathcal{L}(F)+d\left(J^{\prime \prime}\right) / 2-\partial e\left(J^{\prime \prime}\right) / 2$ is in $C_{0}(\Gamma(X), \mathbf{Z}) \cap \partial C_{1}(\Gamma(X), \mathbf{R})=\partial C_{1}(\Gamma(X), \mathbf{Z})$. We conclude that there exists $\xi$ in $C_{1}(\Gamma(X), \mathbf{Z})$, unique up to translation by $H_{1}(\Gamma(X), \mathbf{Z})$, such that

$$
\operatorname{deg} \mathcal{L}(F)=\partial\left(\xi+e\left(J^{\prime \prime}\right) / 2\right)-d\left(J^{\prime \prime}\right) / 2 \text {. }
$$

Consider the Delony polyhedron $D$ in $K(\Gamma(X))$ defined by

$$
D=\left\{\xi+\sum_{j \in J^{\prime \prime}} t_{j} e_{j} ; 0 \leqslant t_{j} \leqslant 1 \text { for } j \in J^{\prime \prime}\right\} .
$$

Then $D$ is determined by $F$ uniquely up to the translation by $H_{1}(\Gamma(X), \mathrm{Z})$ and we have $b(D)=\xi+e\left(J^{\prime \prime}\right) / 2, \operatorname{deg} \mathcal{L}(F)=\partial b(D)-d\left(J^{\prime \prime}\right) / 2$ and Supp $D=J^{\prime \prime}$. Thus we get (3), (4) and (5). Since $\{F \in \operatorname{LB}(X)$; Supp $\bar{D}(F)=$ $\left.J^{\prime \prime}\right\}=\operatorname{Pic}\left(X\left(J-J^{\prime \prime}\right)\right)$ by Proposition 10.1, we get (1) by applying known results for the Picard group to the curve $X\left(J-J^{\prime \prime}\right)$. We postpone the proof of (2) until we come to the precise definition of "closure" in terms of a functor (cf. §12, Proposition 12.10).

11. Stable and semistable line bundles. Let $\phi$ be an element of $\partial C_{1}(\Gamma(X), \mathbf{R})$. We defined in $\$ 6$ the notions of $\phi$-semistable and $\phi$-stable Delony polyhedra, and defined subsets $K_{\phi}(\Gamma(X)) \supset K_{\phi}^{0}(\Gamma(X)) \supset K_{\phi \text {-stable }}(\Gamma(X))$ which are invariant under the translation by $H_{1}(\Gamma(X), Z)$. Except for the middle set, they are subcomplexes of $K(\Gamma(X))$.

Definimon. For $\phi$ in $\partial C_{1}(\Gamma(X), \mathbf{R})$, we denote by $\bar{K}_{\phi}(\Gamma(X)), \bar{K}_{\phi}^{0}(\Gamma(X))$ and $\bar{K}_{\phi \text {-stable }}(\Gamma(X))$ the quotient of these subsets with respect to the translation action of $H_{1}(\Gamma(X), \mathrm{Z})$.

REMARK. $\bar{K}_{\phi}(\Gamma(X)), \bar{K}_{\phi}^{0}(\Gamma(X))$ and $\bar{K}_{\phi \text {-stable }}(\Gamma(X))$ are finite sets. Indeed first of all $\bar{K}^{0}(\Gamma(X))$ is in one to one correspondence with the quotient of the Namikawa decomposition $\operatorname{Del}_{\phi}\left(H^{1}(\Gamma(X), \mathbf{R})\right)$ by the translation action of $\rho\left(H_{1}(\Gamma(X), \mathbf{Z})\right)$, secondly $\bar{K}_{\phi-s t a b l e}(\Gamma(X))$ is a subset of this, and finally there is a finite-to-one map $\mu: K_{\phi}(\Gamma(X)) \rightarrow K_{\phi}^{0}(\Gamma(X))$. 
By means of the surjective map $\bar{D}: \mathrm{LB}^{0}(X) \rightarrow \bar{K}(T(X))$ we defined in Theorem 10.5, we now introduce the notions of semistability and stability for line bundles.

Defintion. Let $\phi$ be in $\partial C_{1}(\Gamma(X), \mathbf{R})$. A line bundle $F$ in $\mathrm{LB}^{0}(X)$ is called $\phi$-semistable (resp. $\phi$-stable) if $\bar{D}(F)$ is in $\bar{K}_{\phi}(\Gamma(X))$ (resp. $\bar{K}_{\phi \text {-stable }}(\Gamma(X))$ ). We denote by $\mathrm{LB}_{\phi}^{0}(X), \mathrm{LB}_{\phi}^{00}(X)$ and $\mathrm{LB}_{\phi-\text { stable }}^{0}(X)$ the set of isomorphism classes of $\phi$-semistable line bundles, those line bundles with $\bar{D}(F)$ in $\bar{K}_{\phi}^{0}(T(X))$, and $\phi$-stable line bundles, respectively.

By Propositions 6.4 and 6.6, we have:

Proposition 11.1. Let $\phi$ be in $\partial C_{1}(\Gamma(X), \mathbf{R})$. Then a line bundle $F$ in $\operatorname{LB}^{0}(X)$ is $\phi$-semistable (resp. $\phi$-stable) if and only if $\partial \bar{D}(F) \subset \phi+\partial V_{J}(0)$ (resp. $\partial \bar{D}(F) \subset \phi+$ rel.int $\left.\partial V_{J}(0)\right)$.

By Corollary 7.2, we have:

Proposition 11.2. (i) For $A$ in $\operatorname{Arr}(\Gamma(X))$, the sets $\bar{K}_{\phi}(\Gamma(X))$, $\bar{K}_{\phi}^{0}(\Gamma(X))$, $\bar{K}_{\phi-\text { stable }}(T(X)), \mathrm{LB}_{\phi}^{0}(X), \mathrm{LB}_{\phi}^{00}(X)$ and $\mathrm{LB}_{\phi-\text { stable }}^{0}(X)$ stay the same as long as $\phi$ is in $\partial e(J) / 2+\operatorname{rel}$.int $A$.

(ii) For $\xi$ in $C_{1}(\Gamma(X), Z)$ and $\phi$ in $\partial C_{1}(\Gamma(X), \mathbf{R})$, the translation by $\xi$ induces bijections

$$
\bar{K}_{\phi}(\Gamma(X)) \stackrel{\sim}{\rightarrow} \bar{K}_{\phi+\partial \xi}(\Gamma(X)), \quad \bar{K}_{\phi}^{0}(\Gamma(X)) \stackrel{\sim}{\rightarrow} K_{\phi+\partial \xi}^{0}(\Gamma(X)) .
$$

and

$$
\bar{K}_{\phi-\text { stable }}(\Gamma(X)) \stackrel{\sim}{\rightarrow} \bar{K}_{\phi+\partial \xi \text { stable }}(\Gamma(X)) .
$$

By Proposition 7.6, we have:

Proposition 11.3.

$$
\operatorname{LB}_{\phi}^{0}(X)=\operatorname{LB}_{\phi-s t a b l e}^{0}(X)
$$

if and only if $\phi$ is nondegenerate, i.e. $\phi-\partial e(J) / 2$ is in the interior of a top-dimensional polyhedron in $\operatorname{Arr}(\Gamma(X))$.

We now construct algebraic schemes $\mathrm{Jac}_{\phi \text {-stable }}(X)$ and $\mathrm{Jac}_{\phi}(X)$, the closed point sets of which are $\mathrm{LB}_{\phi-\text { stable }}^{0}(X)$ and a quotient set of $\mathrm{LB}_{\phi}^{0}(X)$, respectively. The technique we employ is the geometric invariant theory of Mumford [20]. The situation we encounter is surprisingly similar to that encountered by Seshadri [32] in the case of vector bundles on a nonsingular curve. We postpone until a later section the analysis of the equivalence relation defined by the quotient map $\mathrm{LB}_{\phi}^{0}(X) \rightarrow \mathrm{Jac}_{\phi}(X)(\operatorname{Spec} k)$.

Let us choose locally free line bundles $\left\{M_{i}\right\}_{i \in I}$ on $X$ such that

$$
\operatorname{deg}_{\tilde{X}_{i}}\left(\theta_{\tilde{X}_{i}} \otimes_{\theta_{X}} M_{i^{\prime}}\right)=\delta_{i i^{\prime}}
$$


For $n=\sum_{i \in I} n_{i} v_{i}$ in $C_{0}(\Gamma(X), \mathrm{Z})$, we define a locally free line bundle on $X$ by

$$
\mathcal{O}_{X}(n)=\bigotimes_{i \in I} M^{\otimes n_{i}}
$$

In particular, $\theta_{X}(n)$ is ample if $n_{i}>0$ for all $i$ in $I$. For a coherent $\theta_{X}$-module $G$, we have

$$
\chi(G(n))=\sum_{i \in I} \operatorname{rank}_{X_{i}}(G) n_{i}+\chi(G) .
$$
G.

Definition. We call $P(n)=\chi(G(n))$ the generalized Hilbert polynomial of

Let $\phi$ be in $\partial C_{1}(\Gamma(X), \mathbf{R})$. We have seen in the remark at the beginning of this section that the complex $\bar{K}_{\phi}(\Gamma(X))$ is finite. Moreover, $F$ in $\operatorname{LB}_{\phi}^{0}(X)$ has the following properties: $\bar{D}(F)$ is in $\bar{K}_{\phi}(\Gamma(X))$ and the sequence

$$
0 \rightarrow F \rightarrow \mathcal{L}(F) \rightarrow \bigoplus_{j \in J(F)} k\left(Q_{j}\right) \rightarrow 0
$$

is exact, where $\mathcal{L}(F)=\left(\mathcal{E}_{i}(F)\right)_{i \in I}$ is in $\operatorname{Pic}(\tilde{X})$ with $\operatorname{deg} \mathcal{L}(F)=\partial b(\bar{D}(F))$ $d(\operatorname{Supp} \bar{D}(F)) / 2$ and $J(F)=J-\operatorname{Supp} \bar{D}(F)$. We easily see from these facts that there exists a positive integer $\theta$ such that $\operatorname{deg}_{\tilde{X}_{i}}\left(\mathcal{L}_{i}(F)\right) \geqslant-\theta$ for all $F$ in $\operatorname{LB}_{\phi}^{0}(X)$ and all $i$ in $I$.

Thus $\mathrm{LB}_{\phi}^{0}(X)$ is contained in the family $B_{\theta}$ consisting of isomorphism classes of $\theta_{X}$-modules $F^{\prime}$ which have an exact sequence of the form

$$
0 \rightarrow F^{\prime} \rightarrow \bigoplus_{i \in I} L_{i} \rightarrow \bigoplus_{j \in J^{\prime}} k\left(Q_{j}\right) \rightarrow 0
$$

for a subset $J^{\prime}$ of $J$ and for $L_{i}$ in $\operatorname{Pic}\left(\tilde{X}_{i}\right)$ with $\operatorname{deg}_{\tilde{X}_{i}}\left(L_{i}\right) \geqslant-\theta$ for all $i$ in $I$.

Obviously $B_{\theta}$ is a bounded family, hence for $\tilde{n}=\sum \tilde{n}_{i} v_{i}$ in $C_{0}(\Gamma(X), \mathbf{Z})$ with $\tilde{n}_{i}$ large enough for all $i$ in $I, H^{0}\left(F^{\prime}(n)\right)$ generates $F^{\prime}(n)$ and $H^{1}\left(F^{\prime}(n)\right)=0$ for all $F^{\prime}$ in $B_{\theta}$. For details see Ishida [36].

We fix such $\tilde{n}$. Then for $F$ in $\operatorname{LB}^{0}(X)$, the generalized Hilbert polynomial of $F(\tilde{n})$ is given by

$$
P(n)=\chi(F(\tilde{n})(n))=[v(I), n+\tilde{n}]+\chi\left(\theta_{X}\right) .
$$

Take a vector space $E$ over $k$ with

$$
\operatorname{dim} E=P(0)=[v(I), \tilde{n}]+\chi\left(\theta_{X}\right) .
$$

Then $F(\tilde{n})$ is a quotient $\theta_{X}$-module of $\theta_{X} \otimes_{k} E$ for all $F$ in $\operatorname{LB}_{\phi}^{0}(X)$.

Consider $Q(E / P)=\operatorname{Quot}\left(\theta_{X} \otimes_{k} E / P\right)$, Grothendieck's scheme parametrizing all quotient $\theta_{X}$-modules $G$ of $\theta_{X} \otimes_{k} E$ with $\chi(G(n))=P(n)$. It is easy to modify the proof in [FGA, Exposé 221] to show that $Q(E / P)$ is a projective algebraic scheme over $k$. For a closed point $q$ in $Q(E / P)$, we denote by $G_{q}$ the corresponding quotient

$$
\vartheta_{x} \otimes_{k} E \rightarrow G_{q} \rightarrow 0
$$


Let $R(E / P) \subset Q(E / P)$ be the subset consisting of $q$ with

$$
\left\{\begin{array}{l}
G_{q} \text { torsion-free, } \\
E \stackrel{\sim}{\rightarrow} H^{0}\left(G_{q}\right) \text { is an isomorphism, } \\
H^{1}\left(G_{q}\right)=0 .
\end{array}\right.
$$

Then $R(E / P)$ is obviously a GL(E)-invariant open subset (cf. Corollary in the Appendiix) of $Q(E / P)$ with respect to the canonical action of $G L(E)$. Moreover, $G_{q}$ is isomorphic to $G_{q^{\prime}}$ if and only if $q$ and $q^{\prime}$ are in the same $\mathrm{GL}(E)$-orbit.

For $q$ in $R(E / P)$, there exists a unique $F$ in $\operatorname{LB}^{0}(X)$ such that $G_{q}=$ $F(\tilde{n})$. Let

$$
R_{\phi}(E / P) \supset R_{\phi \text {-stable }}(E / P)
$$

be the open subsets of $R(E / P)$ consisting of $q$ with $G_{q}=F(\tilde{n})$ for $F$ in $\mathrm{LB}_{\phi}^{0}(X)$ and $\mathrm{LB}_{\phi-\text { stable }}^{0}(X)$, respectively. We now come to our main existence theorem.

Theorem 11.4. (1) $A$ good quotient $\mathrm{Jac}_{\phi}(X)=R_{\phi}(E / P) / \mathrm{GL}(E)$ exists. Two points $q$ and $q^{\prime}$ in $R_{\phi}(E / P)$ go into the same point of $\mathrm{Jac}_{\phi}(X)$ if and only if the closures of $\mathrm{GL}(E)$-orbits of $q$ and $q^{\prime}$ intersect.

(2) $\mathrm{Jac}_{\phi}(X)$ is a projective algebraic scheme.

(3) $\mathrm{Jac}_{\phi}(X)$ is reduced.

(4) The restriction of the above quotient induces a geometric quotient

$$
R_{\phi-\text { stable }}(E / P) \rightarrow \mathrm{Jac}_{\phi-\text { stable }}(X)
$$

which is a principal PGL $(E)$-bundle, locally trivial in the Zariski topology.

(5) The universal quotient sheaf $G$ on $X \times R_{\phi-s t a b l e}(E / P)$ satisfies the following property: there exists an invertible sheaf $M^{\prime}$ on $R_{\phi-s a b l e}(E / P)$ such that $P_{2}^{*} M^{\prime} \otimes G$ descends to $X \times \mathrm{Jac}_{\phi-\text { stable }}(X)$.

Proof. By Proposition 11.2 (i), we may assume that $\phi$ has rational coefficients, i.e. is in $\partial C_{1}(\Gamma(X), \mathbf{Q})$. For $\tilde{n}$ in $C_{0}(T(X), \mathbf{Z})$, we define $\lambda=$ $\lambda(\tilde{n}, \phi)=\Sigma_{i \in I} \lambda_{i} v_{i}$ by

$$
\lambda_{i}=\left\{\left[v_{i}, \tilde{n}-d(J) / 2+\phi\right]+\chi\left(\theta_{\tilde{X}_{i}}\right)\right\} / P(0)
$$

where

$$
P(0)=[v(I), \tilde{n}]+\chi\left(\theta_{X}\right)=\sum_{i \in I} \chi\left(\theta_{\tilde{x}_{i}}\right)+[v(I), \tilde{n}-d(J) / 2]
$$

by Lemma 4.2. If $\tilde{n}_{i}=\left[v_{i}, \tilde{n}\right]$ is large enough, then $\lambda_{i}$ is a positive rational number for all $i$ in $I$. Moreover, we see that $\sum_{i \in I} \lambda_{i}=[v(I), \lambda]=1$, since $[v(I), \phi]=0$.

We now choose a positive integer $N$ such that $N_{i}=\lambda_{i} N$ are integers for all $i$. Then let us choose $N$ smooth points $x_{1}, \ldots, x_{N}$ of $X$ so that $N_{i}$ of them are 
on the component $X_{i}$ for all $i$ in $I$. Then we have a GL(E)-morphism

$$
\tau: R(E / P) \rightarrow \mathbf{P}(E)^{N}
$$

by assigning to $q$ in $R(E / P)$ the point $\tau(q)=\left(\tau_{\alpha}(q)\right)_{1<\alpha<N}$ where $\tau_{\alpha}(q)$ is the point of the projective space $\mathbf{P}(E)$ defined by the one-dimensional quotient

$$
\tau_{\alpha}(q): E \rightarrow G_{q}\left(x_{\alpha}\right)=\text { the fiber of } G_{q} \text { at } x_{\alpha} .
$$

Let $Z=\mathbf{P}(E)^{N}$, and we consider the stability and semistability of its points with respect to the standard action of $\operatorname{SL}(E)$ introduced by Mumford [20]. According to his theory, a point $z=\left(z_{\alpha}\right)_{1<\alpha<N}$ of $Z$ with the one-dimensional quotient $z_{\alpha}: E \rightarrow E_{\alpha}$ is semistable (resp. stable) if and only if for all nonzero proper subspaces $E^{\prime}$ of $E$, we have

$$
\sum_{1<\alpha<N} \operatorname{dim} z_{\alpha}\left(E^{\prime}\right) / N \geqslant \operatorname{dim} E^{\prime} / \operatorname{dim} E \quad(\text { resp. }>)
$$

(see also Seshadri [32]).

We denote by $Z^{s s}$ and $Z^{s}$ the open subsets of $Z$ consisting of semistable and stable points, respectively. Then we have the following basic existence theorem in the geometric invariant theory (Mumford [20] and Seshadri [32], [33]):

THEOREM. Regardless of the characteristic of $k, a$ good quotient $Z^{\text {ss }} / \mathrm{GL}(E)$ and a geometric quotient $Z^{s} / \mathrm{GL}(E)$ exist. Moreover, $Z^{s s} / \mathrm{GL}(E)$ is projective, $Z^{s} / \mathrm{GL}(E)$ is its open subscheme and $Z^{s} \rightarrow Z^{s} / \mathrm{GL}(E)$ is a principal PGL(E)-bundle, locally trivial in the Zariski topology.

In general, if there is a proper injective $\mathrm{GL}(E)$-morphism from a scheme $Y$ to $Z^{s s}$ which sends an open subscheme $Y^{\prime}$ of $Y$ to $Z^{s}$, and if a good quotient $Z^{s s} / \mathrm{GL}(E)$ and a geometric quotient $Z^{s} / \mathrm{GL}(E)$ exist, then a good quotient $Y / \mathrm{GL}(E)$ and a geometric quotient $Y^{\prime} / \mathrm{GL}(E)$ exist. This is the so-called method of covariant. See Mumford [20] and Ramanathan [35, Lemma 4.1].

Thus (1), (2) and (4) of Theorem 11.4 are consequences of the following:

Proposition 11.5. Given $\phi$ in $\partial C_{1}(T(X), Q)$, we can find $\tilde{n}$ in $C_{0}(\Gamma(X), Z)$ with $\left[v_{i}, \tilde{n}\right]$ sufficiently large for all $i$, a large enough positive integer $N$ so that $\lambda_{i}(\tilde{n}, \phi) N=N_{i}$ are positive integers for all $i$, and an ordered set $\left(x_{\alpha}\right)_{1<\alpha<N}$ of $N$ smooth points on $X$ with $N_{i}$ of them on the component $X_{i}$ for all $i$ in $I$ such that

(i) the morphism $\tau: R(E / P) \rightarrow Z=P(E)^{N}$ is injective,

(ii) $\tau\left(R_{\phi}(E / P)\right)$ is contained in $Z^{s s}$, and for $q$ in $R_{\phi}(E / P)$, the point $\tau(q)$ is in $Z^{s}$ if and only if $q$ is in $R_{\phi-s t a b l e}(E / P)$, and

(iii) the induced morphism $\tau: R_{\phi}(E / P) \rightarrow Z^{s s}$ is proper (even a closed immersion). 
Proof. (i) can be proved by the "diagonal" argument as in Seshadri [32]. For the proof of (ii), we need the following lemmas, the first of which is obvious:

LEMMA 11.6. For a nonempty proper subset $I^{\prime}$ of $I$ and $F$ in $\mathrm{LB}(X)$, we denote by $S_{I}(F)$ the subsheaf of germs of sections of $F$ which vanish completely on the subcurve $\cup_{i \in I^{\prime}} X_{i}$ of $X$. Then there exists an exact sequence of $\theta_{X}$-modules

$$
0 \rightarrow S_{I^{\prime}}(F) \rightarrow F \rightarrow \bigoplus_{i \in I^{\prime}} \mathcal{E}_{i}(F) \rightarrow \underset{j \in J(F)\left(I^{\prime}\right)}{\bigoplus^{\prime}} k\left(Q_{j}\right) \rightarrow 0,
$$

where $J(F)\left(I^{\prime}\right)$ is the set of edges $j$ in $J(F)$ both of whose end points are in $I^{\prime}$. In particular, we have

$$
\chi\left(S_{I^{\prime}}(F)\right)=\chi(F)-\sum_{i \in I^{\prime}} \chi\left(\mathcal{E}_{i}(F)\right)+\left|J(F)\left(I^{\prime}\right)\right| .
$$

LEMMA 11.7. Let $F$ be in $\operatorname{LB}^{0}(X)$. Then $F$ is $\phi$-semistable (resp. $\phi$-stable) if and only if for every nonempty proper subset $I^{\prime}$ of $I$, we have

$$
\left[v\left(I-I^{\prime}\right), \lambda(\tilde{n}, \phi)\right] \chi(F(\tilde{n})) \geqslant \chi\left(S_{I^{\prime}}(F(\tilde{n}))\right) \quad(\text { resp. }>) \text {. }
$$

Proof. We know that $\chi(F(\tilde{n}))=P(0)$. Hence by the definition of $\lambda(\tilde{n}, \phi)$, the left-hand side is equal to $\left[v\left(I-I^{\prime}\right), \tilde{n}-d(J) / 2+\phi\right]+\sum_{i \in I-I^{\prime}} \chi\left(\theta_{\tilde{x}_{i}}\right)$. Let us now compute the right-hand side using Lemma 11.6. First of all, we have

$$
\begin{aligned}
\left|J(F(\tilde{n}))\left(I^{\prime}\right)\right| & =\left|J(F)\left(I^{\prime}\right)\right| \\
& =\left[v\left(I^{\prime}\right), d(J(F)) / 2\right]-\left(\delta_{J(F)} v\left(I^{\prime}\right), \delta_{J(F)} v\left(I^{\prime}\right)\right) / 2
\end{aligned}
$$

by Corollary 4.4. Secondly by the Riemann-Roch theorem and Theorem 10.5 (4), we have

$$
\begin{aligned}
-\sum_{i \in I^{\prime}} \chi\left(\mathcal{E}_{i}(F(\tilde{n}))\right)= & {\left[v\left(I^{\prime}\right),-\partial b(\bar{D}(F))+d(J-J(F)) / 2-\tilde{n}\right] } \\
& -\sum_{i \in I^{\prime}} \chi\left(\Theta_{\tilde{X}_{i}}\right) .
\end{aligned}
$$

Since $\chi(F(\tilde{n}))=P(0)=[v(I), \tilde{n}-d(J) / 2]+\sum_{i \in I} \chi\left(\theta_{\tilde{X}_{i}}\right)$, we conclude that

$$
\begin{aligned}
\chi\left(S_{I^{\prime}}(F(\tilde{n}))\right)=[ & \left.v\left(I-I^{\prime}\right), \tilde{n}-d(J) / 2\right]-\left[v\left(I^{\prime}\right), \partial b(\bar{D}(F))\right] \\
& +\sum_{i \in I-I^{\prime}} \chi\left(\theta_{\tilde{X_{i}}}\right)-\left(\delta_{J(F)} v\left(I^{\prime}\right), \delta_{J(F)} v\left(I^{\prime}\right)\right) / 2 .
\end{aligned}
$$

Note that $v(I)=v\left(I^{\prime}\right)+v\left(I-I^{\prime}\right)$ and $\delta_{J(F)} v(I)=0$. Hence the left-hand side of the lemma is not less than (resp. greater than) the right-hand side if and only if

$$
\left[v\left(I-I^{\prime}\right), \partial b(\bar{D}(F))-\phi\right] \leqslant\left(\delta_{J(F)} v\left(I-I^{\prime}\right), \delta_{J(F)} v\left(I-I^{\prime}\right)\right) / 2
$$

(resp. <). Thus by Corollary 6.3 and the remark after that applied to the graph $\{I, J(F)\}$, we see that the inequalities of the lemma are satisfied for all 
nonempty proper subsets $I^{\prime}$ of $I$ if and only if $\partial b(\bar{D}(F))-\phi$ is contained in $\partial V_{J(F)}(0)$ (resp. in rel.int $\partial V_{J(F)}(0)$ ). By Propositions 6.4 and 6.6 we are done.

We now continue the proof of Proposition 11.5. We have chosen $\tilde{n}$ so that $E \stackrel{\sim}{\rightarrow} H^{0}\left(G_{q}\right)$ and $H^{1}\left(G_{q}\right)=0$ for all $q$ in $R(E / P)$. Thus $\chi\left(G_{q}\right)=\operatorname{dim} E$ and $\chi\left(S_{I^{\prime}}\left(G_{q}\right)\right)=\operatorname{dim} E_{I^{\prime}}$, where $E_{I^{\prime}}$ is the subspace of $E=H^{0}\left(G_{q}\right)$ consisting of sections which vanish completely on the subcurve $\cup_{i \in I^{\prime}} X_{i}$. If $\tilde{n}_{i}$ is large enough for all $i$, we see easily that $E_{I^{\prime}}$ generates $S_{I^{\prime}}\left(G_{q}\right)$.

For a subspace $E^{\prime}$ of $E$ and a smooth point $x$ of $X$, let us denote by $E^{\prime}(x)$ the image of $E^{\prime}$ by the quotient linear map $E \rightarrow G_{q}(x)=$ the fiber of $G_{q}$ at $x$, hence its dimension is either 0 or 1 .

Then by definition, $\operatorname{dim} E_{I^{\prime}}\left(x_{\alpha}\right)=0$ or 1 , according as $x_{\alpha}$ is in $\cup_{i \in I^{\prime}} X_{i}$ or not. Since $N_{i}=\lambda_{i} N$ is the number of points $x_{\alpha}$ on the component $X_{i}$, we get $\left[v\left(I-I^{\prime}\right), \lambda(\tilde{n}, \phi)\right]=\sum_{i \in I-I^{\prime}} N_{i} / N=\sum_{1 \leqslant \alpha \leqslant N} \operatorname{dim} E_{I^{\prime}}\left(x_{\alpha}\right) / N$. Thus by Lemma 11.7, $q$ is contained in $R_{\phi}(E / P)$ (resp. $R_{\phi \text {-stable }}(E / P)$ ), if and only if

$$
\sum_{1<\alpha<N} \operatorname{dim} E_{I^{\prime}}\left(x_{\alpha}\right) / N \geqslant \operatorname{dim} E_{I^{\prime}} / \operatorname{dim} E \quad \text { (resp. }>\text { ) }
$$

is satisfied for all nonempty proper subsets $I^{\prime}$ of $I$.

To prove (ii) of Proposition 11.5, we need to show that for $\tilde{n}$ and $N$ large and properly chosen, the inequality

$$
\left.\sum_{1<\alpha<N} \operatorname{dim} E^{\prime}\left(x_{\alpha}\right) / N \geqslant \operatorname{dim} E^{\prime} / \operatorname{dim} E \quad \text { (resp. }>\right)
$$

is satisfied for any nonzero proper subspace $E^{\prime}$ of $E$, if the same inequality is satisfied for all subspaces of the form $E^{\prime}=E_{I^{\prime}}$ for a nonempty proper subset $I^{\prime}$ of $I$.

For $F$ in $\mathrm{LB}^{0}(X)$ with $G_{q}=F(\tilde{n})$, we have an exact sequence

$$
0 \rightarrow G_{q} \rightarrow \bigoplus_{i \in I} L_{i} \rightarrow \bigoplus_{j \in J(F)} k\left(Q_{j}\right) \rightarrow 0
$$

where $L_{i}=\mathcal{L}_{i}(F)\left(\tilde{n}_{i}\right) \in \operatorname{Pic}\left(\tilde{X}_{i}\right)$. Given a nonzero proper subspace $E^{\prime}$ of $E$, let $I^{\prime}$ be the subset of $I$ consisting of $i$ for which the composed map $E^{\prime} \rightarrow \theta_{\tilde{X}_{i}}$ $\otimes_{k} E^{\prime} \rightarrow L_{i}$ is zero. Thus $E^{\prime}$ is a subspace of $E_{I^{\prime}}$. If $E^{\prime}=E_{I^{\prime}}$, then automatically $I^{\prime}$ is a nonempty proper subset, and there is nothing to prove. We now suppose $E^{\prime} \neq E_{I^{\prime}}$. Then for $i$ in $I-I^{\prime}$, we have a nonzero map $\theta_{\tilde{X}_{i}} \otimes_{k} E^{\prime} \rightarrow$ $L_{i}$. Let $T_{i}$ be the cokernel, which is a torsion $\theta_{\tilde{X}}$-module. Obviously $\left|\operatorname{Supp}\left(T_{i}\right)\right|$ $\leqslant \operatorname{deg}_{\tilde{X}_{i}}\left(L_{i}\right)$. But $\sum_{i \in I} \operatorname{deg}_{\tilde{X}_{i}}\left(L_{i}\right) v_{i}=\operatorname{deg} \mathcal{L}(F(\tilde{n}))=\tilde{n}+\operatorname{deg} \mathcal{L}(F)$ assumes only a finite number of admissible values for $q$ in $R_{\phi}(E / P)$. Hence $\operatorname{deg}_{\tilde{X}_{i}}\left(L_{i}\right)$ is bounded above by a constant $c$ depending on $\tilde{n}$. Thus for $i$ in $I-I^{\prime}$, we get

$$
\begin{aligned}
\sum_{x_{\alpha} \in X_{i}} \operatorname{dim} E^{\prime}\left(x_{\alpha}\right) & \geqslant N_{i}-\left|\operatorname{Supp}\left(T_{i}\right)\right| \geqslant N_{i}-\operatorname{deg}_{\tilde{X}_{i}}\left(L_{i}\right) \\
& \geqslant N_{i}-c .
\end{aligned}
$$


Hence

$$
\begin{aligned}
& \sum_{1<\alpha<N} \operatorname{dim} E^{\prime}\left(x_{\alpha}\right) / N-\operatorname{dim} E^{\prime} / \operatorname{dim} E \\
& \geqslant \sum_{i \in I-I^{\prime}}\left(N_{i}-c\right) / N-\operatorname{dim} E^{\prime} / \operatorname{dim} E \\
&=\sum_{1<\alpha<N} \operatorname{dim} E_{I^{\prime}}\left(x_{\alpha}\right) / N-c\left|I-I^{\prime}\right| / N-\operatorname{dim} E^{\prime} / \operatorname{dim} E \\
& \geqslant\left(\operatorname{dim} E_{I^{\prime}}-\operatorname{dim} E^{\prime}\right) / \operatorname{dim} E-c\left|I-I^{\prime}\right| / N,
\end{aligned}
$$

which is positive if $N$ is large enough, since $\operatorname{dim} E_{I^{\prime}} \geqslant \operatorname{dim} E^{\prime}$ by assumption.

For the proof of Proposition 11.5 (iii), we proceed as on p. 363 of Seshadri [32]. For $q$ in $Q(E / P), G_{q}$ is a quotient of $\theta_{X} \otimes_{k} E$ with the generalized Hilbert polynomial $\chi\left(G_{q}(n)\right)=P(n)=[v(I), n]+P(0)$. In particular, $G_{q}$ has rank one on each component of $X$. Let $T_{q}$ be the torsion part of $G_{q}$. Let $\tilde{G}_{q}=G_{q} / T_{q}$, which is in $\operatorname{LB}(X)$. We extend $\tau: R(E / P) \rightarrow Z$ to a multivalued map

$$
\tilde{\tau}: Q(E / P) \rightarrow Z=\mathbf{P}(E)^{N}
$$

by setting $\tilde{\tau}(q)=\left(\tilde{\tau}_{\alpha}(q)\right)_{1<\alpha<N}$ with

$$
\tilde{\tau}_{\alpha}(q)= \begin{cases}E \rightarrow G_{q}\left(x_{\alpha}\right) & \text { if } x_{\alpha} \notin \operatorname{Supp}\left(T_{q}\right), \\ \text { arbitrary one-dimensional quotient of } E & \text { if } x_{\alpha} \in \operatorname{Supp}\left(T_{q}\right) .\end{cases}
$$

We now show that $\tilde{n}$ and $N$ can be so chosen that they are large enough and that for $q$ in $Q(E / P)$ not in $R_{\phi}(E / P), \tilde{\tau}(q)$ is not in $Z^{s}$. Note first that $\operatorname{dim} H^{0}\left(T_{q}\right)$ is absolutely bounded. Indeed, $\left\{G_{q} ; q \in Q(E / P)\right\}$ is a bounded family. Hence $n_{0}$ can be so chosen that $H^{0}\left(G_{q}\left(n_{0}\right)\right)$ generates $G_{q}\left(n_{0}\right)$ and $H^{1}\left(G_{q}\left(n_{0}\right)\right)=0$. Then $\operatorname{dim} H^{0}\left(T_{q}\right) \leqslant \operatorname{dim} H^{0}\left(G_{q}\left(n_{0}\right)\right)=P\left(n_{0}\right)$.

When $q$ is not in $R_{\phi}(E / P)$, we need to show that there exists a nonzero proper subspace $E^{\prime}$ of $E$ such that

$$
\sum_{1<\alpha<N} \operatorname{dim} E^{\prime}\left(x_{\alpha}\right) / N<\operatorname{dim} E^{\prime} / \operatorname{dim} E
$$

if $\tilde{n}$ and $N$ are properly chosen, where $E^{\prime}\left(x_{\alpha}\right)$ is again the image of $E^{\prime}$ by the quotient map $\tilde{\tau}_{\alpha}(q)$. Consider

$$
K=\operatorname{ker}\left[E \rightarrow H^{0}\left(G_{q}\right) \rightarrow H^{0}\left(\tilde{G}_{q}\right)\right] .
$$

Case (1). If $K \neq 0$, we take $E^{\prime}=K$. Then obviously $\sum_{1<\alpha<N} \operatorname{dim} E^{\prime}\left(x_{\alpha}\right) \leqslant$ $\left|\operatorname{Supp}\left(T_{q}\right)\right|<\operatorname{dim} H^{0}\left(T_{q}\right)$ is absolutely bounded above. Hence

$$
\sum_{1<\alpha<N} \operatorname{dim} E^{\prime}\left(x_{\alpha}\right) / N \leqslant \operatorname{Supp}\left(T_{q}\right) / N,
$$

which is less than $\operatorname{dim} E^{\prime} / \operatorname{dim} E$ if $N$ is large enough.

Case (2). If $K=0$, the composed map $E \rightarrow H^{0}\left(G_{q}\right) \rightarrow H^{0}\left(\tilde{G}_{q}\right)$ is injective. 
In particular, $E \rightarrow H^{0}\left(G_{q}\right)$ is injective. Since $\tilde{G}_{q}$ is a line bundle, we have an exact sequence

$$
0 \rightarrow \tilde{G}_{q}(-\tilde{n}) \rightarrow \bigoplus_{i \in I} L_{i}^{\prime} \rightarrow \bigoplus_{j \in J^{\prime}} k\left(Q_{j}\right) \rightarrow 0
$$

for $L_{i}^{\prime}$ in $\operatorname{Pic}\left(\tilde{X}_{i}\right)$ and a subset $J^{\prime}$ of $J$.

Case (2a). If $\operatorname{deg}_{\tilde{X}_{i}}\left(L_{i}^{\prime}\right) \geqslant-\theta$ for all $i$, then, by what we said immediately after Proposition 11.3, $\tilde{G}_{q}(-\tilde{n})$ is contained in $B_{\theta}$. By our choice of $\tilde{n}$, we thus have $H^{1}\left(\tilde{G}_{q}\right)=H^{1}\left(G_{q}\right)=0$. Hence $\operatorname{dim} H^{0}\left(G_{q}\right)=\chi\left(G_{q}\right)=P(0)=\operatorname{dim} E$ and $E \stackrel{\sim}{\rightarrow} H^{0}\left(G_{q}\right) \supset H^{0}\left(T_{q}\right)$. If $H^{0}\left(T_{q}\right) \neq 0$, we let $E^{\prime}$ be the inverse image of $H^{0}\left(T_{q}\right)$ in $E$. We then proceed as in Case (1). Thus we may assume $H^{0}\left(T_{q}\right)=0$, i.e. $T_{q}=0$ and $\tilde{G}_{q}=G_{q}$. This means that $q$ is in $R(E / P)$. Since $q$ is not in $R_{\phi}(E / P)$ by assumption, there exists, by what we saw in the proof of (ii), a nonempty proper subset $I^{\prime}$ of $I$ such that $\Sigma_{1<\alpha<N} \operatorname{dim} E_{I}\left(x_{\alpha}\right) / N<$ $\operatorname{dim} E_{I^{\prime}} / \operatorname{dim} E$. Hence $\tilde{\tau}(q)$ is not semistable in $Z$.

Case (2b). Let $I^{\prime}$ be the subset of $I$ defined by

$$
I^{\prime}=\left\{i \in I ; \operatorname{deg}_{X_{i}}\left(L_{i}^{\prime}\right)<-\theta\right\} .
$$

We may assume $I^{\prime}$ to be nonempty. Since $E$ generates $G_{q}$, hence $\tilde{G}_{q}, E$ generates $L_{i}^{\prime}\left(\tilde{n}_{i}\right)$ generically for all $i$. Thus we get

$$
0 \leqslant \operatorname{deg}_{\tilde{X}_{i}}\left(L_{i}^{\prime}\left(\tilde{n}_{i}\right)\right) \leqslant \tilde{n}_{i}-\theta
$$

for $i$ in $I^{\prime}$. Let $E^{\prime}$ be the subspace of $E$ consisting of elements which go, by the composed map $E \rightarrow H^{0}\left(G_{q}\right) \rightarrow H^{0}\left(\tilde{G}_{q}\right)$, to the subspace of sections of $\tilde{G}_{q}$ vanishing completely on the subcurve $\cup_{i \in I^{\prime}} X_{i}$. Thus $E^{\prime}$ is the kernel of the composed map $E \rightarrow H^{0}\left(\tilde{G}_{q}\right) \rightarrow \bigoplus_{i \in I^{\prime}} H^{0}\left(L_{i}^{\prime}\left(\tilde{n}_{i}\right)\right)$. But the dimension of the image of this composed map is bounded above by $\Sigma_{i \in I^{\prime}} \operatorname{dim} H^{0}\left(L_{i}^{\prime}\left(\tilde{n}_{i}\right)\right)$, which is bounded above by

$$
\begin{aligned}
\sum_{i \in I^{\prime}} \operatorname{deg}_{\tilde{X}_{i}}\left(L_{i}^{\prime}\left(\tilde{n}_{i}\right)\right)+c^{\prime} & <\sum_{i \in I^{\prime}}\left(\tilde{n}_{i}-\theta\right)+c^{\prime} \\
& =\left[v\left(I^{\prime}\right), \tilde{n}\right]-\left|I^{\prime}\right| \theta+c^{\prime},
\end{aligned}
$$

for an absolute constant $c^{\prime}$. Since $\operatorname{dim} E=P(0)=[v(I), \tilde{n}]+\chi\left(\theta_{X}\right)$, we get $\operatorname{dim} E^{\prime}>\left[v\left(I-I^{\prime}\right), \tilde{n}\right]+\chi\left(\theta_{X}\right)+\left|I^{\prime}\right| \theta-c^{\prime}$. On the other hand since $E^{\prime}\left(x_{\alpha}\right)=\mathrm{C}$ unless $x_{\alpha}$ is in $\cup_{i \in I-I^{\prime}} X_{i}$ or in $\operatorname{Supp}\left(T_{q}\right)$, we have

$$
\begin{aligned}
\sum_{1<\alpha<N} \operatorname{dim} E^{\prime}\left(x_{\alpha}\right) & \leqslant \sum_{i \in I-I^{\prime}} N_{i}+\left|\operatorname{Supp}\left(T_{q}\right)\right| \\
= & \sum_{i \in I-I^{\prime}} \lambda_{i}(\tilde{n}, \phi) N+\left|\operatorname{Supp}\left(T_{q}\right)\right| \\
= & \left\{\left[v\left(I-I^{\prime}\right), \tilde{n}-d(J) / 2+\phi\right]+\sum_{i \in I-I^{\prime}} \chi\left(\theta_{\tilde{x}_{i}}\right)\right\} N / P(0) \\
& +\left|\operatorname{Supp}\left(T_{q}\right)\right| .
\end{aligned}
$$


Suppose for a moment that $\tilde{\tau}(q)$ is semistable. Then we should have

$$
\sum_{1<\alpha<N} \operatorname{dim} E^{\prime}\left(x_{\alpha}\right) / N \geqslant \operatorname{dim} E^{\prime} / \operatorname{dim} E .
$$

Hence from what we saw above, we get

$$
\begin{aligned}
\left|\operatorname{Supp}\left(T_{q}\right)\right| / N+\{ & {\left.\left[v\left(I-I^{\prime}\right), \tilde{n}-d(J) / 2+\phi\right]+\sum_{i \in I-I^{\prime}} \chi\left(\theta_{\bar{X}_{i}}\right)\right\} / P(0) } \\
& \geqslant \sum_{1<\alpha<N} \operatorname{dim} E^{\prime}\left(x_{\alpha}\right) / N \geqslant \operatorname{dim} E^{\prime} / \operatorname{dim} E \\
& >\left\{\left[v\left(I-I^{\prime}\right), \tilde{n}\right]+\chi\left(\theta_{X}\right)+\left|I^{\prime}\right| \theta-c^{\prime}\right\} / P(0) .
\end{aligned}
$$

Then we have

$$
\begin{aligned}
P(0)\left|\operatorname{Supp}\left(T_{q}\right)\right| / N+[v(I & \left.\left.-I^{\prime}\right),-d(J) / 2+\phi\right] \\
& +\sum_{i \in I-I^{\prime}} \chi\left(\theta_{\tilde{X}_{i}}\right)-\chi\left(\theta_{X}\right)+c^{\prime}>\left|I^{\prime}\right| \theta .
\end{aligned}
$$

Since $P(0)=[v(I), \tilde{n}]+\chi\left(\theta_{X}\right)$ and $\left|\operatorname{Supp}\left(T_{q}\right)\right|$ is absolutely bounded above, we may choose $N$ and $\tilde{n}$ so that they are large enough and moreover the inequality

$$
P(0) \cdot\left|\operatorname{Supp}\left(T_{q}\right)\right| / N<1
$$

is satisfied. If we choose $\theta$, as we may, to be greater than $1+[v(I-$ $\left.\left.I^{\prime}\right),-d(J) / 2+\phi\right]+c^{\prime}+\sum_{i \in I-I^{\prime}} \chi\left(\theta_{\tilde{X}_{i}}\right)-\chi\left(\theta_{X}\right)$, we have a contradiction, since $\left|I^{\prime}\right| \geqslant 1$.

See Ishida [36] for the proof of the fact that $\tau$ can even be made a closed immersion.

For the proof of Theorem 11.4 (5), we imitate Mumford-Newstead [25]. Since we deal with rank one sheaves, our proof is much simpler than theirs. For simplicity, we denote $R_{\phi \text {-stable }}(E / P)=R$ and $\operatorname{Jac}_{\phi \text {-stable }}(X)=J$ in this proof, since there is no confusion of them with our previous notations here. Fix a smooth point $x$ of $X$. Then the restriction $G \mid x \times R$ of the universal quotient sheaf $G$ on $X \times R$ to $x \times R$ can be identified with an invertible sheaf on $R$, since $G \mid X \times q$ is locally free at $x \times q$ for all $q$ in $R$. Let $M^{\prime}$ be the invertible sheaf on $R$ dual to $G \mid x \times R$. GL(E) acts on $X \times R$ trivially on the first factor and in the standard way on the second factor. Then $G$ has a natural GL(E)-linearization compatible with the GL(E)-action on $X \times R$. The center $G_{m}$ of $\mathrm{GL}(E)$ acts trivially on $X \times R$ and by scalar multiplication on $G$. On the other hand, $M^{\prime}$ has the induced $G L(E)$-linearization, in which $G_{m}$ acts by the scalar multiplication of the inverse. Therefore, $G^{\prime}=P_{2}^{*} M^{\prime} \otimes$ $G$ has a $G L(E)$-linearization in which $G_{m}$ acts trivially. We thus conclude that $G^{\prime}$ has a $\operatorname{PGL}(E)$-linearization. Since we know that $R \rightarrow J$ is a principal PGL(E)-bundle by (4), this linearization is equivalent to descent data on $G^{\prime}$ 
with respect to $X \times R \rightarrow X \times J$. By the fundamental theorem of the descent theory in [FGA, Expose 190], $G^{\prime}$ descends to a sheaf on $X \times J$.

It remains to show Theorem $11.4(3)$, i.e. $\operatorname{Jac}_{\phi}(X)$ is reduced. Since it is a good quotient of $R_{\phi}(E / P)$, it is enough to show that $R_{\phi}(E / P)$ is reduced.

For simplicity, let us denote $R=R_{\phi}(E / P)$ in this proof. Consider the product $R \times \mathbf{P}\left(E^{*}\right)$. Let $Y$ be its subset consisting of points $(q, u)$ with

$$
q: \mathcal{O}_{X} \otimes_{k} E \rightarrow G_{q} \text { surjective, } u: E^{*} \rightarrow k \text { surjective, }
$$

such that the composite map

$$
r: \Theta_{X} \stackrel{1 \otimes u^{*}}{\rightarrow} \theta_{X} \otimes_{k} E \stackrel{q}{\rightarrow} G_{q}
$$

is injective with Coker $(r)$ having support consisting of $d=[v(I), \tilde{n}]$ distinct points of $X$. Consider the projection $P_{1}: Y \rightarrow R$. By definition, $q$ induces an isomorphism $E \stackrel{\sim}{\rightarrow} H^{0}\left(G_{q}\right), H^{1}\left(G_{q}\right)=0$ and $G_{q}$ is in $\operatorname{LB}_{\phi}^{0}(X)(\tilde{n})$. Since $\tilde{n}_{i}$ was chosen large enough for all $i$ in $I$, we see immediately that $P_{1}(Y)=R$.

Let Hilb ${ }_{X}^{d}$ be the Hilbert scheme of $X$ parametrizing 0-dimensional subschemes $D$ of $X$ with $\chi\left(\theta_{D}\right)=d=[v(I), \tilde{n}]$. Consider the map

$$
w: Y \rightarrow \text { Hilb }_{X}^{d}
$$

which assigns to $(q, u)$ in $Y$ the dual of $r$

$$
r^{*}=(1 \otimes u) \circ q^{*}: G_{q}^{*}=\mathcal{J}_{\operatorname{Com}_{\theta_{X}}}\left(G_{q}, \theta_{X}\right) \rightarrow \theta_{X} .
$$

Since Coker $(r)$ has support consisting of $d$ distinct points, we see that $r^{*}$ is injective with $\operatorname{Coker}\left(r^{*}\right)$ consisting of $d$ distinct points. Obviously, the image $w(Y)=H$ is the open subset of Hilb ${ }_{X}^{d}$ parametrizing reduced 0-cycles $D$ on $X$ of degree $d$, whose ideal sheaf $I_{D}$ has the property $I_{D}^{*} \in \mathrm{LB}_{\phi}^{0}(X)(-\tilde{n})$.

LEMMA 11.8. (i) $Y$ is an open subscheme of $R \times \mathbf{P}\left(E^{*}\right)$.

(ii) $w: Y \rightarrow H$ is a formally smooth morphism.

(iii) $H$ is reduced.

From this lemma, we conclude that $R=R_{\phi}(E / P)$ is reduced. Indeed, we have

$$
\begin{aligned}
& R \times \mathrm{P}\left(E^{*}\right) \\
& \cup \text { open } \\
& R \stackrel{P_{1}}{\longleftarrow} Y \stackrel{w}{\longrightarrow} H \subset \operatorname{Hilb}_{X}^{d}
\end{aligned}
$$

with $P_{1} \mid Y$ and $w$ formally smooth and with $H$ reduced. Hence $R$ is necessarily reduced.

ProOf OF Lemma 11.8. Let $S$ be a noetherian $k$-scheme and let $(q, u)$ be an $S$-valued point of $R \times \mathbf{P}\left(E^{*}\right)$, i.e. $q: \theta_{x \times S} \otimes_{k} E \rightarrow G$ is a surjection with an 
$S$-flat $\theta_{X \times S}$-module $G$ and $u: \theta_{S} \otimes_{k} E^{*} \rightarrow L$ is a surjection with an invertible sheaf $L$ on $S$. Consider the composite map

$$
r: \theta_{X \times S} \stackrel{1 \otimes u^{*}}{\rightarrow} L^{-1} \otimes_{\theta_{S}} \theta_{X \times S} \otimes_{k} E \stackrel{q}{\rightarrow} G
$$

and its dual

$$
r^{*}: G^{*}=\mathcal{H}_{\operatorname{Com}_{\theta_{x} s}}\left(G, \theta_{X \times s}\right) \rightarrow \theta_{x \times s} .
$$

we know by the Lemma in the Appendix that the images of closed points of $S$ by this $S$-valued point are contained in $Y$ if and only if $r$ is injective with $S$-flat cokernel whose support has $d$ components each of which projects isomorphically onto $S$. Hence $Y$ is obviously open, and we get (i).

We identify $Y$ with the open subscheme of $R \times \mathbf{P}\left(E^{*}\right)$ whose $S$-valued points have the property that $r$ is injective with $S$-flat cokernel whose support has $d$ components each projecting isomorphically onto $S$. In this case the restriction of the dual $r^{*}$ to each fiber is injective. Hence again by the Lemma in the Appendix we conclude that $r^{*}$ is injective with $S$-flat cokernel whose support has $d$ components each projecting isomorphically onto $S$. Thus $r^{*}$ defines an $S$-valued point of the open subscheme $H$ of Hilb ${ }_{X}^{d}$, hence $w$ is a morphism. We now show (ii). Let $S^{\prime}$ be a noetherian $k$-scheme and let $S$ be a closed subscheme of $S^{\prime}$ defined by a nilpotent ideal sheaf. Let $D^{\prime}$ be an $S^{\prime}$-valued point of $H$, hence a subscheme of $X \times S^{\prime}$ finite and flat over $S^{\prime}$, each of its $d$ components projecting isomorphically onto $S^{\prime}$, and, moreover, its defining ideal $I^{\prime}$ having the property that its restriction to each fiber $X \times s^{\prime}$ belongs to $\operatorname{LB}_{\phi}^{0}(X)(-\tilde{n})$. On the other hand, let $(q, u)$ be an $S$-valued point of $Y$, i.e. a surjection $q: \theta_{X \times S} \otimes_{k} E \rightarrow G$ with $S$-flat $G$ and a surjection $u: \theta_{S} \otimes_{k} E^{*} \rightarrow L$ with an invertible sheaf $L$ on $S$ such that $r^{*}: G^{*} \rightarrow \theta_{X \times S}$ coincides with the restriction of $I^{\prime} \hookrightarrow \theta_{X \times S^{\prime}}$ to $X \times S$. By the Corollary in

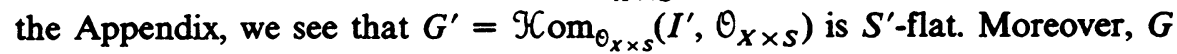
is the restriction of $G^{\prime}$ to $X \times S$. Consider the projection

$$
P_{2}: X \times S^{\prime} \rightarrow S^{\prime} \text {. }
$$

Since $H^{1}\left(G^{\prime} \mid X \times s^{\prime}\right)=0$ for all closed points $s^{\prime}$ of $S^{\prime}$, we see that $R^{1} P_{2}^{*} G^{\prime}=$ 0 , and $P_{2}^{*} G^{\prime}$ commutes with base change (cf. Mumford [21]). Thus the restriction of $P_{2}^{*} G^{\prime}$ to $S$ coincides with $P_{2}^{*} G$. Since we may assume $S^{\prime}$ to be local, it is easy to see that the isomorphism

$$
\vartheta_{X} \otimes_{k} E \stackrel{\sim}{\rightarrow} P_{2}^{*} G
$$

extends to an isomorphism

$$
\Theta_{S^{\prime}} \otimes_{k} E \stackrel{\sim}{\rightarrow} P_{2}^{*} G^{\prime}
$$

Obviously, we have thus a surjective homomorphism $q^{\prime}: \theta_{X \times s^{\prime}} \otimes_{k} E \rightarrow G^{\prime}$ which lifts $q$, and a surjective homomorphism $u^{\prime}: \theta_{S^{\prime}} \otimes_{k} E \rightarrow L^{\prime}$ with an 
invertible sheaf $L^{\prime}$ on $S^{\prime}$ which lifts $u$. Thus we have (ii).

It remains to show (iii), i.e. $H$ is reduced. But by definition, $H$ parametrizes subschemes $D$ of $X$ consisting of $d$ distinct points of $X$. Thus there exists a surjective map $\sigma: U \rightarrow H$ from the open subset $U$ of the $d$ th Cartesian product $X^{d}=X \times \cdots \times X$ consisting of points $x=\left(x_{1}, \ldots, x_{d}\right)$ with $x_{i} \neq$ $x_{j}$ for $i \neq j$. Since $\sigma$ is actually the quotient map with respect to the action of the symmetric group of degree $d$, and since $U$ is reduced, we conclude that $H$ is reduced.

Thus we conclude the proof of Lemma 11.8, hence Theorem 11.4.

REMARK. It is much easier to show that $\operatorname{Jac}_{\phi \text {-stable }}(X)$ is reduced. In fact, the completion of the local rings at its closed points are of the form

$$
k\left[\left[x_{1}, x_{1}^{\prime}, \ldots, x_{r}, x_{r}^{\prime}, y_{1}, \ldots, y_{s}\right]\right] /\left(x_{1} x_{1}^{\prime}, \ldots, x_{r} x_{r}^{\prime}\right)
$$

Indeed, since $\operatorname{Jac}_{\phi \text {-stable }}(X)$ represents a functor, as we see below, we can easily show that there is a formally smooth surjective morphism $H^{\prime} \rightarrow$ $\operatorname{Jac}_{\phi \text {-stable }}(X)$ for an open subscheme $H^{\prime}$ of $H$, which is reduced by Lemma 11.8 (iii). This is the method employed by D'Souza [7], when $X$ is irreducible, or more generally when $\phi$ is nondegenerate, $\operatorname{since} \operatorname{Jac}_{\phi}(X)=\operatorname{Jac}_{\phi \text {-stable }}(X)$ in this case by Proposition 11.3.

12. Families of line bundles and presentations. In this section, we consider flat families of line bundles on $X$ parametrized by a $k$-scheme $S$. Then we will be able to interpret schemes $\operatorname{Jac}_{\phi}(X)$ and $\mathrm{Jac}_{\phi \text {-stable }}(X)$ in the previous section in terms of the functors they represent.

We note first the following: If $S$ is the spectrum of a field, then we define line bundles on $X \times S$ as torsion-free everywhere rank one $\theta_{X \times S}$-modules. Then for a line bundle $F$ on $X \times S$, we can define, as in $\S 10$, the subset $J(F)$ consisting of those $j$ in $J$ for which $F$ is locally free at $Q_{j} \times S$. The invertible sheaf $\mathcal{L}(F)$ on $\tilde{X} \times S$ is defined as $\theta_{\tilde{X}} \otimes_{\theta_{X}} F / \theta_{\tilde{X} \times S}$-torsion. Then the definition of $\bar{D}(F), \mathrm{LB}(X \times S), \mathrm{LB}_{\phi}^{0}(X \times S), \mathrm{LB}_{\phi}^{00}(X \times S)$ and $\mathrm{LB}_{\phi-\text { stable }}^{0}(X$ $X S)$ is an immediate consequence of these observations.

Let $\phi$ be an element of $\partial C_{1}(\Gamma(X), \mathbf{R})$, which will be fixed throughout this section.

DEFINITION. We denote by $W^{*}$ the contravariant functor from the category of $k$-schemes to that of sets defined as follows: for a $k$-scheme $S, W^{*}(S)$ is the set of isomorphism classes of coherent $\theta_{X \times S}$-modules $F$, which are $S$-flat and whose restrictions $F_{s}$ to $X \times s$, for closed points $s$ of $S$, are line bundles, i.e. $F_{s} \in \mathrm{LB}(X \times s)$. For a $k$-morphism $S^{\prime} \rightarrow S$, the map $W^{*}(S) \rightarrow W^{*}\left(S^{\prime}\right)$ is defined by the pull-back $\theta_{S^{\prime}} \otimes_{\theta_{S}} F$. We denote by $W_{\phi}^{*}$ (resp. $W_{\phi \text {-stable }}^{*}$ ) the subfunctors for which $W_{\phi}^{*}(S)$ (resp. $W_{\phi \text {-stabie }}^{*}(S)$ ) consists of $F$ with $F_{s} \in$ $\mathrm{LB}_{\phi}^{0}(X \times s)\left(\right.$ resp. $\operatorname{LB}_{\phi \text {-stable }}^{0}(X \times s)$ ) for all closed points $s$ of $S$. 
$W^{*} \supset W_{\phi}^{*} \supset W_{\phi-s t a b l e}^{*}$ are presheaves of sets in the Zariski topology of the category of $k$-schemes.

DEFINITION. We denote by $W, W_{\phi}$ and $W_{\phi \text {-stable }}$ the sheafification of $W^{*}$, $W_{\phi}^{*}$ and $W_{\phi \text {-stable, }}^{*}$ respectively, in the Zariski topology.

For a $k$-scheme $S$, we can easily see that

$$
\begin{aligned}
W(S)= & W^{*}(S) / \operatorname{Pic}(S), \quad W_{\phi}(S)=W_{\phi}^{*}(S) / \operatorname{Pic}(S), \\
& W_{\phi \text {-stable }}(S)=W_{\phi \text {-stable }}^{*}(S) / \operatorname{Pic}(S),
\end{aligned}
$$

where $\operatorname{Pic}(S)$ acts on $W^{*}(S)$ by tensor product $P_{2}^{*} M \otimes F$ for an invertible sheaf $M$ on $S$ and $F$ in $W^{*}(S)$. Alternatively, for a fixed smooth point $x$ of $X$, we can think of $W(S)$ as the set of elements $F$ in $W^{*}(S)$ endowed with a rigidification $F \mid x \times S \stackrel{\sim}{\rightarrow} \Theta_{S}$.

Note that $W(\operatorname{Spec} k)=\mathrm{LB}(X), \quad W_{\phi}(\operatorname{Spec} k)=\mathrm{LB}_{\phi}^{0}(X)$ and $W_{\phi \text {-stable }}$ by tensor product $F \mapsto P_{2}^{*} L \otimes F$ for $L$ in $\operatorname{Pic}_{X}^{0}(S)$ and $F$ in $W(S)$. We see easily that we have bijections

$$
W_{\phi} / \mathrm{Pic}_{X}^{0} \stackrel{\sim}{\rightarrow} \bar{K}_{\phi}(\Gamma(X)), \quad W_{\phi-\text { stable }} / \mathrm{Pic}_{X}^{0} \stackrel{\sim}{\rightarrow} \bar{K}_{\phi \text {-stable }}(\Gamma(X)) .
$$

Convention. As in the beginning of $\S 10$, we identify a module on $\tilde{X} \times S$ with its direct image by $\tilde{X} \times S \rightarrow X \times S$. For a closed point $Q$ on $\tilde{X}$, we denote by $[Q]$ the divisor on $\tilde{X}$ defined by the point. $\theta_{\tilde{X}}([Q])$ is the invertible sheaf on $\tilde{X}$ defined by the divisor $[Q]$. For an $\theta_{X}$-module $F$ and $j$ in $J$, we denote by $F\left(Q_{j}\right)$ the restriction of $F$ to $Q_{j} \times S \leftarrow S$. If $L$ is an $\theta_{\tilde{X}}$-module, then we have a canonical decomposition

$$
L\left(Q_{j}\right)=L\left(Q_{j}^{+}\right) \oplus L\left(Q_{j}^{-}\right),
$$

where $L\left(Q_{j}^{+}\right)$and $L\left(Q_{j}^{-}\right)$are the restrictions of $L$ to $Q_{j}^{+} \times S \leftarrow S$ and $Q_{j}^{-} \times S \leftarrow S$, respectively. When $S=\operatorname{Spec} R$ is affine, we denote by $R\left(Q_{j}\right)$ the structure sheaf on $Q_{j} \times S \leftarrow S$.

Definition. Let $S$ be a $k$-scheme. A presentation $a: L \rightarrow N=\bigoplus_{j \in J^{\prime}} N_{j}$ over $S$ is a surjective $\theta_{X \times S}$-homomorphism from an invertible sheaf $L$ on $\tilde{X} \times S$ to a direct $\operatorname{sum} N=\bigoplus_{j \in J^{\prime}} N_{j}$ of invertible sheaves $N_{j}$ on $Q_{j} \times S$ for a subset $J^{\prime}$ of $J$. A morphism from a presentation $a: L \rightarrow N$ to another $a^{\prime}$ :

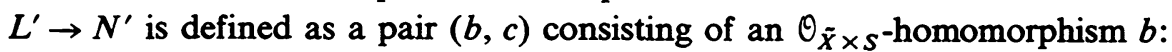

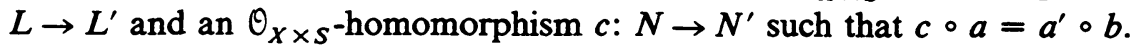

Definition. We denote by Pres*(?) the contravariant functor from the category of $k$-schemes to that of sets defined as follows: for a $k$-scheme $S$, $\operatorname{Pres}^{*}(S)$ is the set of isomorphism classes of presentations over $S$. For a $k$-morphism $S^{\prime} \rightarrow S$, the map $\operatorname{Pres}^{*}(S) \rightarrow \operatorname{Pres}^{*}\left(S^{\prime}\right)$ is defined by the pullback under $X \times S^{\prime} \rightarrow X \times S$ in an obvious way. For a subset $J^{\prime}$ of $J$ and $m$ in $C_{0}(\Gamma(x), \mathbf{Z})$, we denote by $\operatorname{Pres}^{*}\left(m, J^{\prime} ;\right.$ ?) the subfunctor of Pres*(?) defined as follows: for a $k$-scheme $S$, $\operatorname{Pres}^{*}\left(m, J^{\prime} ; S\right)$ is the set of isomorphism classes of presentations $a: L \rightarrow N$ such that deg $L_{s}=m$ for all closed 
points $s$ of $S$ and that $N$ is the direct sum $N=\bigoplus_{j \in J^{\prime}} N_{j}$ of invertible sheaves $N_{j}$ on $Q_{j} \times S$.

It is immediate to see that $F=\operatorname{ker}(a)$ for a presentation $a: L \rightarrow N$ is a flat family of line bundles on $X$ parametrized by $S$, i.e. $F \in W^{*}(S)$. Thus we have a functorial morphism

$$
\text { ker: } \operatorname{Pres}^{*}(S) \rightarrow W^{*}(S) \text {. }
$$

Definition. We denote by $\operatorname{Pres}(?)$ and $\operatorname{Pres}\left(m, J^{\prime} ;\right.$ ?) the sheafifications defined by

$$
\begin{aligned}
\operatorname{Pres}(S) & =\operatorname{Pres}^{*}(S) / \operatorname{Pic}(S), \\
\operatorname{Pres}\left(m, J^{\prime} ; S\right) & =\operatorname{Pres}^{*}\left(m, J^{\prime} ; S\right) / \operatorname{Pic}(S),
\end{aligned}
$$

where $\operatorname{Pic}(S)$ acts on $\operatorname{Pres}{ }^{*}(S)$ by the tensor product

$$
1 \otimes a: P_{2}^{*} M \otimes_{\Theta_{\tilde{x} \times s}} L \rightarrow P_{2}^{*} M \otimes_{\theta_{x \times s}} N
$$

for $M$ in $\operatorname{Pic}(S)$ and $a: L \rightarrow N$ in $\operatorname{Pres}^{*}(S)$.

Thus there is a canonical morphism of functors

$$
\text { ker: Pres(?) } \rightarrow W(?) \text {. }
$$

Definition. For $m$ in $C_{0}(\Gamma(X), \mathbf{Z})$, we denote by Pic $_{\tilde{X}}^{m}$ the connected component of the Picard scheme Pic $\tilde{X}$ of $\tilde{X}$ consisting of those invertible sheaves $L$ on $\tilde{X}$ with $\operatorname{deg} L=m$. We denote by $\mathscr{P}_{m}$ the universal Poincaré invertible sheaf on $\tilde{X} \times \operatorname{Pic}_{\tilde{X}}^{m}$ determined up to the tensor product of an invertible sheaf on $\mathrm{Pic}_{\tilde{X}}^{m}$.

Proposition 12.1. The functor Pres $\left(m, J^{\prime} ;\right.$ ?) is represented by the $\Pi_{j \in J^{\prime}} \mathbf{P}_{1^{-}}$ bundle over Pic $\bar{X}_{\bar{X}}^{m}$, obtained as the fiber product over $\mathbf{P i c}_{\bar{X}}^{m}$ of $\mathbf{P}_{1}$-bundles $\mathbf{P}\left(\mathscr{P}_{m}\left(Q_{j}^{+}\right) \oplus \mathscr{P}_{m}\left(Q_{j}^{-}\right)\right)$over $\operatorname{Pic}_{X}^{m}$ for $j$ in $J^{\prime}$.

Henceforth, we identify $\operatorname{Pres}\left(m, J^{\prime} ;\right.$ ?) with this fiber product.

Proof. Let $a: L \rightarrow N=\bigoplus_{j \in J^{\prime}} N_{j}$ be a presentation over $S$. Then by restriction onto $Q_{j} \times S \leftarrow S$, we have a surjective $\theta_{S}$-homomorphism

$$
a\left(Q_{j}\right): L\left(Q_{j}\right)=L\left(Q_{j}^{+}\right) \oplus L\left(Q_{j}^{-}\right) \rightarrow N_{j},
$$

which gives rise to a section of the $\mathbf{P}_{1}$-bundle $\mathbf{P}\left(L\left(Q_{j}^{+}\right) \oplus L\left(Q_{j}^{-}\right)\right)$over $S$ for each $j$ in $J^{\prime}$. The rest of the proof is an immediate consequence of the universality of $\mathscr{P}_{m}$.

Definition. We denote by $\operatorname{StPres}(?)$ and $\operatorname{StPres}\left(m, J^{\prime} ;\right.$ ?) the subfunctors of Pres(?) and Pres( $m, J^{\prime} ;$ ?), respectively, defined as follows: for a $k$-scheme $S$, $\operatorname{StPres}(S)$ consists of strict presentations, i.e. presentations $a: L \rightarrow N=$ $\bigoplus_{j \in J^{\prime}} N_{j}$ for which the restriction of $a\left(Q_{j}\right)$ to $L\left(Q_{j}^{+}\right)$and $L\left(Q_{j}^{-}\right)$are both surjective onto $N_{j}$ for each $j$ in $J^{\prime}$.

Definition. For a free Z-module $A$ of finite rank, we denote by $G_{m} \otimes A$ the algebraic torus over $k$ defined as the spectrum of the group algebra over $k$ 
of the dual Z-module of $A$, i.e. the algebraic torus whose character group is dual to $A$.

Corollary 12.2. StPres $\left(m, J^{\prime} ;\right.$ ?) is represented by the $G_{m} \otimes C_{1}\left(\left\{I, J^{\prime}\right\}\right.$, Z)-bundle over $\mathrm{Pic}_{\bar{X}}^{m}$ obtained as the fiber product over $\mathrm{Pic}_{\tilde{X}}^{m}$ of the $G_{m}$-bundles

$$
\delta \operatorname{pec}\left(\bigoplus_{\nu \in \mathbf{Z}}\left(\mathscr{P}_{m}\left(Q_{j}^{+}\right) \otimes_{\Theta_{s}} \mathscr{P}_{m}\left(Q_{j}^{-}\right)^{-1}\right)^{\otimes_{\nu}}\right) \text {, }
$$

which is obtained by deleting the zero and infinite sections from the $\mathbf{P}_{1}$-bundle $\mathbf{P}\left(\mathscr{P}_{m}\left(Q_{j}^{+}\right) \oplus \mathscr{P}_{m}\left(Q_{j}^{-}\right)\right)$, for $j$ in $J^{\prime}$.

The proof of this corollary is immediate from the definition of strictness.

Henceforth, we also identify $\operatorname{StPres}\left(m, J^{\prime} ;\right.$ ?) with this fiber product.

We have an exact sequence of $\mathbf{Z}$-modules

$$
C_{0}\left(\left\{I, J^{\prime}\right\}, \mathbf{Z}\right) \stackrel{\delta_{J^{\prime}}}{\rightarrow} C_{1}\left(\left\{I, J^{\prime}\right\}, \mathbf{Z}\right) \rightarrow H^{1}\left(\left\{I, J^{\prime}\right\}, \mathbf{Z}\right) \rightarrow 0,
$$

thus we have an exact sequence of algebraic tori

$$
G_{m} \otimes C_{0}\left(\left\{I, J^{\prime}\right\}, \mathbf{Z}\right) \stackrel{\delta_{J^{\prime}}}{\rightarrow} G_{m} \otimes C_{1}\left(\left\{I, J^{\prime}\right\}, \mathbf{Z}\right) \rightarrow G_{m} \otimes H^{1}\left(\left\{I, J^{\prime}\right\}, \mathbf{Z}\right) \rightarrow 1 .
$$

On the other hand, $G_{m} \otimes C_{0}\left(\left\{I, J^{\prime}\right\}, \mathbf{Z}\right)$ can be thought of as the automorphism scheme $\operatorname{Aut}_{\theta_{\tilde{X}}}(L)$ of an invertible sheaf $L$ on $\tilde{X}$, where for $i$ in $I$, the $i$ th factor $G_{m}$ acts as the scalar multiplication on the restriction $L \mid \tilde{X}_{i}$ of $L$ on the irreducible component $\tilde{X}_{i}$ of $\tilde{X}$. Then obviously the induced action of $G_{m} \otimes$ $C_{0}\left(\left\{I, J^{\prime}\right\}, Z\right)$ on $\operatorname{StPres}\left(m, J^{\prime} ; ?\right)$ is through the coboundary map

$$
\delta_{J^{\prime}}: G_{m} \otimes C_{0}(\Gamma(X), \mathbf{Z})=G_{m} \otimes C_{0}\left(\left\{I, J^{\prime}\right\}, \mathbf{Z}\right) \rightarrow G_{m} \otimes \delta_{J^{\prime}} C_{0}\left(\left\{I, J^{\prime}\right\}, \mathbf{Z}\right) .
$$

As in Proposition 10.1, we can identify the Picard scheme Pic ${ }_{X\left(J^{\prime}\right)}$ of the partial normalization $\sigma\left(J^{\prime}\right): X\left(J^{\prime}\right) \rightarrow X$ as the universal scheme parametrizing flat families of line bundles $F$ whose restriction $F_{s}$ to each closed fiber satisfies $J\left(F_{s}\right)=J^{\prime}$. For $m$ in $C_{0}\left(\left\{I, J^{\prime}\right\}, \mathbf{Z}\right)=C_{0}(\Gamma(X), \mathbf{Z})$ we denote by $\mathrm{Pic}_{X\left(J^{\prime}\right)}^{\boldsymbol{m}}$ the connected component of $\mathrm{Pic}_{X\left(J^{\prime}\right)}$ consisting of invertible sheaves $L$ on $X\left(J^{\prime}\right)$ whose pull-back by $\tilde{X} \rightarrow X\left(J^{\prime}\right)$ have degree $m$.

Proposition 12.3. The map which assigns $\operatorname{ker}(a)$ to a presentation $a: L \rightarrow N$ gives rise to a morphism

$$
\text { ker: } \operatorname{StPres}\left(m, J^{\prime} ; ?\right) \rightarrow \operatorname{Pic}_{X\left(J^{\prime}\right)}^{m},
$$

by which we can identify the right-hand side as the quotient of the left-hand side by the canonical action of $\operatorname{Aut}_{\theta_{\bar{x}}}\left(\Theta_{\bar{X}}\right)$, or equivalently, as the $G_{m} \otimes$ $H^{1}\left(\left\{I, J^{\prime}\right\}, \mathrm{Z}\right)$-bundle over $\mathrm{Pic}_{\tilde{X}}^{m}$ associated to the left-hand side with respect to the surjective homomorphism $G_{m} \otimes C_{1}\left(\left\{I, J^{\prime}\right\}, \mathbf{Z}\right) \rightarrow G_{m} \otimes H^{1}\left(\left\{I, J^{\prime}\right\}, \mathbf{Z}\right)$.

REMARK. This is a more precise version of Proposition 10.2.

Proof. Let $F$ be a flat family of line bundles on $X$ parametrized by a $k$-scheme $S$ such that $J\left(F_{s}\right)=J^{\prime}$ for all closed points $s$ of $S$, i.e. $F$ is an 
invertible sheaf on $X\left(J^{\prime}\right) \times S$. We denote by $\tilde{F}$ the pull-back of $F$ by $\tilde{X} \times S \rightarrow X\left(J^{\prime}\right) \times S$. Assume further that $\operatorname{deg} \tilde{F}_{s}=m$ for all closed points $s$ of $S$. There is a canonical injective $\theta_{X \times S}$-homomorphism $F \rightarrow \tilde{F}$. Let $\tilde{a}$ be the projection $\tilde{F} \rightarrow \tilde{F} / F$. Then obviously $\tilde{a}$ is a strict presentation. It is easy to see that the map $F \mapsto \tilde{a}$ defines a functorial morphism

$$
\operatorname{Pic}_{X\left(J^{\prime}\right)}^{m}(S) \rightarrow \operatorname{StPres}\left(m, J^{\prime} ; S\right)
$$

which is the right inverse of ker. Let $a: L \rightarrow N$ be a strict presentation over $S$ such that $\operatorname{ker}(a)$ is $\theta_{X \times S}$-isomorphic to $F$. Then the injective $\theta_{X \times S}$-homomorphism $F \stackrel{\sim}{\rightarrow} \operatorname{ker}(a) \rightarrow L$ induces an $\theta_{\tilde{X} \times S}$-isomorphism $\tilde{F} \rightarrow L$. Thus we conclude that the set of isomorphism classes of strict presentations $a$ over $S$ for which $\operatorname{ker}(a) \cong F$ is in one-to-one correspondence with the orbit of $\tilde{a}$ under the action of $\mathrm{Aut}_{\theta_{\tilde{x} \times S}}(\tilde{F})=\left(G_{m} \otimes C_{0}\left(\left\{I, J^{\prime}\right\}, \mathbf{Z}\right)\right)(S)$.

In particular for $J^{\prime}=J$, we get

Corollary 12.4. Let $m$ be in $C_{0}(\Gamma(X), Z)$. Then $\operatorname{Pic}_{X}^{m}$ is the $G_{m} \otimes$ $H^{1}(\Gamma(X), \mathbf{Z})$-bundle over Pic $\bar{X}_{X}^{m}$ associated to the $G_{m} \otimes C_{1}(\Gamma(X), \mathbf{Z})$-bundle $\operatorname{StPres}\left(m, J ;\right.$ ?) with respect to the surjective homomorphism $G_{m} \otimes C_{1}(\Gamma(X), Z)$ $\rightarrow G_{m} \otimes H^{1}(T(X), Z)$. Furthermore, $\operatorname{StPres}(m, J ; ?)$ is obtained as the fiber product over $\mathrm{Pic}_{\tilde{X}}^{m}$ of $G_{m}$-bundles

$$
\operatorname{Spec}\left(\bigoplus_{\nu \in \mathbf{Z}}\left(\mathscr{P}_{m}\left(Q_{j}^{+}\right) \otimes_{\Theta_{x}} \mathscr{P}_{m}\left(Q_{j}^{-}\right)^{-1}\right)^{\otimes_{\nu}}\right)
$$

over $\operatorname{Pic}_{\tilde{X}}^{m}$ for $j$ in $J$.

For $J^{\prime}=J$ and $m=0$, we get:

COROLLARY 12.5. The generalized Jacobian variety $\mathrm{Pic}_{X}^{0}$ is an extension

$$
0 \rightarrow G_{m} \otimes H^{1}(\Gamma(X), \mathbf{Z}) \rightarrow \mathrm{Pic}_{X}^{0} \rightarrow \mathrm{Pic}_{X}^{0} \rightarrow 0
$$

and is obtained as the quotient of $\operatorname{StPres}\left(0, J ;\right.$ ?) by $G_{m} \otimes \delta C_{0}(T(X), Z)$, where the extension

$$
0 \rightarrow G_{m} \otimes C_{1}(\Gamma(X), \mathbf{Z}) \rightarrow \operatorname{StPres}(0, J ; ?) \rightarrow \operatorname{Pic}_{X}^{Q} \rightarrow 0
$$

is obtained as the fiber product over $\mathrm{Pic}_{\tilde{X}}^{0}$ of the $G_{m}$-extensions of $\mathrm{Pic}_{\tilde{X}}^{0}$ corresponding to the invertible sheaves

$$
\mathscr{P}_{0}\left(Q_{j}^{+}\right) \otimes_{\hat{Q}_{\mathrm{Pic} P}} \mathscr{P}_{0}\left(Q_{j}^{-}\right)^{-1}
$$

Proposition 12.3 enables us to describe flat families $F$ of line bundles parametrized by $S$ for which $J\left(F_{s}\right)$ remains constant for closed points $s$ of $S$. To study our functor $W(S)$, however, we need to know also flat families $F$ for which $J\left(F_{s}\right)$ varies from point to point. For this purpose, we next study presentations which need not be strict. 
LEMMA 12.6. Let $S$ be the spectrum of a field. For a presentation a: $L \rightarrow N=\bigoplus_{j \in J^{\prime}} N_{j}$ over $S$, let $F=\operatorname{ker}(a)$. Then we have $J(F) \subset J^{\prime}$ and the

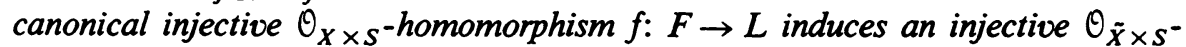
homomorphism $g: \mathcal{L}(F) \rightarrow L$. There are disjoint subsets $J_{+}^{\prime}$ and $J_{-}^{\prime}$ of $J^{\prime}$ such

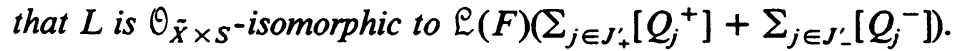

Moreover, $a$ is strict if and only if $L \cong \mathscr{L}(F)$ and $J^{\prime}=J(F)$.

Proof. Without loss of generality, we may assume $S=\operatorname{Spec} k$. Let $j$ be in $J$ and consider the restriction of $f: F \rightarrow L$ to the fiber at $Q_{j}$

$$
f\left(Q_{j}\right): F\left(Q_{j}\right) \rightarrow L\left(Q_{j}\right)=L\left(Q_{j}^{+}\right) \oplus L\left(Q_{j}^{-}\right)
$$

whose cokernel $N\left(Q_{j}\right)$ is either 0 - or 1-dimensional over $k$. In particular $f\left(Q_{j}\right) \neq 0$. If $j$ is in $J(F)$, i.e. $F$ is locally free at $Q_{j}$, then $F\left(Q_{j}\right)$ is 1-dimensional, hence $\operatorname{dim} N\left(Q_{j}\right)=1$, i.e. $j$ is in $J^{\prime}$. Thus we have $J(F) \subset J^{\prime} . f$ induces an $\theta_{\tilde{X}}$-homomorphism $\theta_{\tilde{X}} \otimes_{\theta_{X}} F \rightarrow L$, hence an injective $\theta_{\tilde{X}}$-homomorphism $g$ : $\mathcal{L}(F) \rightarrow L$ by the definition of $\mathcal{L}(F)$ as $\theta_{\tilde{X}} \otimes_{\theta_{X}} F / \theta_{\tilde{X}}$-torsion. Since $g$ is $\Theta_{\tilde{X}}$-linear, we get $g\left(Q_{j}\right)=g\left(Q_{j}^{+}\right) \oplus g\left(Q_{j}^{-}\right)$for $j$ in $J$. The cokernel of $g\left(Q_{j}\right)$ is 0 - or 1-dimensional. In the former case, $g$ is an isomorphism near $Q_{j}$. In the latter case, $j$ is in $J^{\prime}$ and either $g\left(Q_{j}^{+}\right)$is an isomorphism and $g\left(Q_{j}^{-}\right)=0$ or vice versa. Since coker $(f)$, hence coker $(g)$, have 1-dimensional stalk at $Q_{j}$ in this case, we conclude that $L$ is $\theta_{\tilde{X}^{-}}$-isomorphic near $Q_{j}$ to $\mathscr{L}(F)\left(\left[Q_{j}^{-}\right]\right)$or $\mathcal{L}(F)\left(\left[Q_{j}^{+}\right]\right)$, respectively. Thus we are done.

We next study presentations over the spectrum of a discrete valuation ring.

Let $S=\operatorname{Spec} R=\{\eta, s\}$, where $R$ is a discrete valuation ring over $k$ with quotient field $k(\eta)$ and the residue field $k(s)$. Let $\pi$ be a uniformizing parameter for $R$.

Definition. For a flat family of line bundles $F$ in $W(S)$ parametrized by a discrete valuation ring, we denote by $\mathcal{E}(F)$ the double $\theta_{\tilde{X} \times S}$-dual of the pull-back $\theta_{\tilde{X}} \otimes_{\theta_{X}} F$ of $F$ by $\tilde{X} \times S \rightarrow X \times S$.

Since $\tilde{X} \times S$ is a 2-dimnesional regular scheme, it is well known that $\mathcal{L}(F)$ is an invertible sheaf on $\tilde{X} \times S$. There is obviously a canonical injective $\theta_{X \times S}$-homomorphism $f: F \rightarrow \mathcal{L}(F)$.

Proposition 12.7. For a flat family of line bundles $F$ in $W(S)$ parametrized by a discrete valuation ring, the canonical $\Theta_{X \times s}$-homomorphism $\tilde{a}: \mathcal{E}(F) \rightarrow$ $\mathcal{E}(F) / F$ is a presentation with

$$
\mathcal{L}(F) / F \cong \underset{j \in J\left(F_{\eta}\right)}{\bigoplus} R\left(Q_{j}\right)
$$

$\mathcal{E}(F)$ is uniquely determined, up to isomorphism, as the invertible sheaf on $\tilde{X} \times S$ whose restriction to $\tilde{X} \times \eta$ is $\mathcal{L}\left(F_{\eta}\right)$. Moreover, given a presentation a: $L \rightarrow N=\bigoplus_{j \in J^{\prime}} N_{j}$ with $\operatorname{ker}(a) \cong F$, we necessarily have $J\left(F_{\eta}\right) \subset J^{\prime}$ and 


$$
L \cong \mathscr{L}(F)\left(\sum_{j \in J_{+}^{\prime}}\left[Q_{j}^{+}\right]+\sum_{j \in J_{-}^{\prime}}\left[Q_{j}^{-}\right]\right)
$$

for disjoint subsets $J_{+}^{\prime}$ and $J_{-}^{\prime}$ of $J^{\prime}$. The restriction $a_{\eta}$ of a to $X \times \eta$ is strict if and only if $J\left(F_{\eta}\right)=J^{\prime}$ and $L=\mathcal{E}(F)$.

Proof. Our proof is a modification to our situation of Langton's proof in [15] for vector bundles. By the definition of $\mathcal{L}(F)$ as the double dual, we see immediately that $\mathcal{L}(F)_{\eta}$ is isomorphic to $\mathcal{L}\left(F_{\eta}\right)$, which we defined as the quotient modulo torsion of the pull-back of $F_{\eta}$ by $\tilde{X} \times \eta \rightarrow X \times \eta$. Since $\tilde{X}$ is proper and smooth over $k$, a connected component of its Picard scheme $\mathrm{Pic}_{\tilde{X}}$ is proper and separated over $k$. Hence by the valuative criterion, we see that $\mathcal{E}(F)$ is the unique invertible sheaf on $\tilde{X} \times S$ whose restriction to $\tilde{X} \times \eta$ is isomorphic to $\mathcal{L}\left(F_{\eta}\right)$. Let us now consider the restriction $\tilde{a}_{\eta}$ of $\tilde{a}$ to $X \times \eta$. Then we have an exact sequence

$$
0 \rightarrow F_{\eta} \rightarrow \mathcal{L}\left(F_{\eta}\right) \stackrel{\dot{a}_{\eta}}{\rightarrow} \underset{j \in J\left(F_{\eta}\right)}{\bigoplus} k(\eta)\left(Q_{j}\right) \rightarrow 0 .
$$

From what we have seen above, $\mathcal{L}(F)$ can be thought of as an $R$-submodule of the middle term. The image of $\mathcal{L}(F)$ by $\tilde{a}_{\eta}$ is obviously isomorphic to $\bigoplus_{j \in J\left(F_{\eta}\right)} R\left(Q_{j}\right)$. Let

$$
a^{\prime}: \mathcal{L}(F) \rightarrow \bigoplus_{j \in J\left(F_{\eta}\right)} R\left(Q_{j}\right)
$$

be the restriction of $\tilde{a}_{\eta}$ to $\mathcal{L}(F)$. Then $a^{\prime}$ is a presentation over $S$. Let $F^{\prime}=\operatorname{ker}\left(a^{\prime}\right)$, which is $S$-flat and which obviously contains $F$.

We now claim $F=F^{\prime}$. Let $C=F^{\prime} / F$. Then the support of $C$ is obviously contained in $\left\{Q_{j} \times s\right\}_{j \in J}$, hence in particular $C$ is $R$-torsion and $\theta_{X \times s}$-torsion. By tensoring $F^{\prime}$ with the exact sequence

$$
0 \rightarrow \theta_{X \times S} \stackrel{\pi}{\rightarrow} \theta_{X \times S} \rightarrow \theta_{X \times s} \rightarrow 0,
$$

we have an exact sequence

$$
0 \rightarrow \operatorname{Tor}_{1}^{\mathcal{E}_{x \times s}}\left(F^{\prime}, \Theta_{X \times s}\right) \rightarrow F^{\prime} \stackrel{\pi}{\rightarrow} F^{\prime} \rightarrow F_{s}^{\prime} \rightarrow 0 .
$$

Since $F^{\prime}$ is $S$-flat, the multiplication by $\pi$ is an injection on $F^{\prime}$. Hence

$$
\operatorname{Tor}_{1}^{\theta_{x \times s}}\left(F^{\prime}, \theta_{X \times s}\right)=0 .
$$

Then by tensoring $\theta_{X \times s}$ with the exact sequence $0 \rightarrow F \rightarrow F^{\prime} \rightarrow C \rightarrow 0$, we have an exact sequence

$$
0 \rightarrow \operatorname{Tor}_{1}^{\theta_{x \times s}}\left(C, \theta_{X \times s}\right) \rightarrow F_{s} \rightarrow F_{s}^{\prime} \rightarrow C_{s} \rightarrow 0 .
$$

The first term vanishes, since it is obviously $\theta_{X \times s}$-torsion and is contained in $F_{s}$ which is $\theta_{X \times s}$-torsion-free by definition. Thus again by tensoring $\theta_{X \times s}$ with (*), we have an exact sequence $0 \rightarrow C \stackrel{\pi}{\rightarrow} C \rightarrow C_{s} \rightarrow 0$. On the other 
hand, $C$ is $R$-torsion. Thus we have $C=0$. The rest of the proof is similar to that of Lemma 12.6 and is immediate.

From the proof of Proposition 12.7, we get:

COROLlaRY 12.8. Let $S$ be the spectrum of a discrete valuation ring. Then there is a surjective map

ker: $\left\{\right.$ isomorphism classes of presentations a over $S$ with $a_{\eta}$ strict $\} \rightarrow W(S)$ which identifies $W(S)$ as the quotient of the left-hand side with respect to the canonical action of $\delta C_{0}\left(\Gamma(X), R^{*}\right)$, where $R^{*}$ is the multiplicative group of units of $R$.

Again by the proof of Proposition 12.7, the restriction map $a \mapsto a_{\eta}$ from $X \times S$ to $X \times \eta$ gives rise to a bijection

\{isomorphism classes of presentation $a$ over $S$ with $a_{\eta}$ strict $\} \stackrel{\sim}{\rightarrow} \operatorname{StPres}(\eta)$. $W(S)$ is the quotient of the left-hand side by the canonical action of $\delta C_{0}\left(\Gamma(X), R^{*}\right)$, while $W(\eta)$ is the quotient of the right-hand side by the canonical action of $\delta C_{0}\left(\Gamma(X), k(\eta)^{*}\right)$ by Proposition 12.3. The discrete valuation of $R$ gives rise to an exact sequence

$$
1 \rightarrow R^{*} \rightarrow k(\eta)^{*} \stackrel{\text { ord }}{\rightarrow} \mathrm{Z} \rightarrow 0
$$

Thus we have an isomorphism

$$
\text { ord: } \delta C_{0}\left(\Gamma(X), k(\eta)^{*}\right) / \delta C_{0}\left(\Gamma(X), R^{*}\right) \stackrel{\sim}{\rightarrow} \delta C_{0}(\Gamma(X), \mathbf{Z}) \text {. }
$$

Thus we get:

COROLlaRY 12.9. Let $S$ be the spectrum of a discrete valuation ring. Then there is a canonical action of $\delta C_{0}(\Gamma(X), Z)$ on $W(S)$ such that the canonical map $W(S) \rightarrow W(\eta)$ induced by the inclusion $\eta \rightarrow S$ gives rise to a bijection

$$
W(S) / \delta C_{0}(\Gamma(X), \mathbf{Z}) \stackrel{\sim}{\rightarrow} W(\eta) .
$$

REMARK. This corollary shows that the functor $W$ is far from being separated.

Proposition 12.10. Let $S$ be the spectrum of a discrete valuation ring and let $F^{\prime}$ and $F^{\prime \prime}$ be in $\mathrm{LB}^{0}(X \times \eta)$ and $\mathrm{LB}^{0}(X \times s)$, respectively. Then there exist $M^{\prime} \in \operatorname{Pic}^{0}(X \times \eta), M^{\prime \prime} \in \operatorname{Pic}^{0}(X \times s)$ and $F \in W(S)$, such that $F_{\eta} \cong M^{\prime}$ $\otimes_{\Theta_{X \times \eta}} F^{\prime}$ and $F_{s}=M^{\prime \prime} \otimes_{\Theta_{x \times s}} F^{\prime \prime}$ if and only if $\bar{D}\left(F^{\prime}\right)<\bar{D}\left(F^{\prime \prime}\right)$, i.e. the map

$$
\bar{D}: \mathrm{LB}^{0}(X) \rightarrow \bar{K}(\Gamma(X))
$$

defined in Theorem 10.5 is order reversing: $F^{\prime \prime}$ is in the "closure" of the $\mathrm{Pic}^{0}(X)$-orbit of $F^{\prime}$ if and only if $\bar{D}\left(F^{\prime}\right)$ is a face of $\bar{D}\left(F^{\prime \prime}\right)$.

Remark. This is nothing but Theorem 10.5 (2), whose proof we postponed until now. 
Proof. Let $F$ be in $W(S)$. Then by Proposition 12.7, $a: \mathfrak{L}(F) \rightarrow \mathcal{L}(F) / F$ $=\bigoplus_{j \in J\left(F_{\eta}\right)} R\left(Q_{j}\right)$ is a presentation over $S$ with $\operatorname{ker}(a)=F$. Consider its restriction to $X \times s$

$$
a_{s}: \mathcal{L}(F)_{s} \rightarrow \bigoplus_{j \in J\left(F_{\eta}\right)} k(s)\left(Q_{j}\right)
$$

with $\operatorname{ker}\left(a_{s}\right)=F_{s}$. $a_{s}$ need not be strict over $s$. Thus by Lemma 12.6, there exist disjoint subsets $J_{+}^{\prime}$ and $J_{-}^{\prime}$ of $J\left(F_{\eta}\right)$ such that $\mathcal{L}\left(F_{s}\right)$ is $\theta_{\tilde{X} \times s}$-isomorphic to

$$
\mathcal{L}\left(F_{s}\right)\left(\sum_{j \in J_{+}^{\prime}}\left[Q_{j}^{+}\right]+\sum_{j \in J_{-}^{\prime}}\left[Q_{j}^{-}\right]\right) .
$$

In particular, we have $\operatorname{deg} \mathcal{L}\left(F_{s}\right)=\operatorname{deg} \mathfrak{L}(F)_{s}-\Sigma_{i \in I} \mid\left\{j \in J_{+}^{\prime} \amalg J_{-}^{\prime}\right.$; at least one end of $j$ is at $i\} \mid v_{i}$. Moreover, we obviously have $J\left(F_{s}\right)=J\left(F_{\eta}\right)-J_{+}^{\prime}-$ $J_{-}^{\prime}$, hence by Theorem 10.5 (3), we get $\operatorname{Supp} \bar{D}\left(F_{s}\right)=\operatorname{Supp} \bar{D}\left(F_{\eta}\right) \amalg J_{+}^{\prime} \amalg J_{-}^{\prime}$. By Theorem $10.5(4)$, we have $\partial b\left(\bar{D}\left(F_{s}\right)\right)=\operatorname{deg} \mathcal{L}\left(F_{s}\right)+d\left(\operatorname{Supp} \bar{D}\left(F_{s}\right)\right) / 2$ and $\partial b\left(\bar{D}\left(F_{\eta}\right)\right)=\operatorname{deg} \mathcal{L}\left(F_{\eta}\right)+d\left(\operatorname{Supp} \bar{D}\left(F_{\eta}\right)\right) / 2$. Since $\operatorname{deg} \mathcal{L}(F)_{\eta}=$ $\operatorname{deg} \mathcal{L}(F)_{s}$, we see that $\partial b\left(\bar{D}\left(F_{s}\right)\right)-\partial b\left(\bar{D}\left(F_{\eta}\right)\right)=d\left(J_{+}^{\prime} \amalg J_{-}^{\prime}\right) / 2-\Sigma_{i \in I} \mid\{j \in$ $J_{+}^{\prime} \amalg J_{-}^{\prime}$; at least one end of $j$ is at $\left.i\right\}\left|v_{i}=-(1 / 2) \Sigma_{i \in I}\right|\left\{j \in J_{+}^{\prime} \amalg J_{-}^{\prime} ; j\right.$ not a loop and at least one end of $j$ is at $i\} \mid v_{i}$, which is equal to $-\partial\left(e\left(J_{+}^{\prime}\right)-\right.$ $\left.e\left(J_{-}^{\prime}\right)\right) / 2$, since $Q_{j}^{+}$is on $\tilde{X}_{i}$ and $Q_{j}^{-}$is on $\tilde{X}_{i}$, if $j$ is an edge from $i$ to $i^{\prime}$.

Let $D^{\prime}$ be a Delony polyhedron in the $H_{1}(\Gamma(X)$, Z)-equivalence class $\bar{D}\left(F_{\eta}\right)$. Then the polyhedron defined by

$$
D^{\prime \prime}=D^{\prime}+\left\{\sum_{j \in J_{+}^{\prime}} t_{j}\left(-e_{j}\right)+\sum_{j \in J_{-}^{\prime}} t_{j} e_{j} ; 0 \leqslant t_{j} \leqslant 1\right\}
$$

satisfies $D^{\prime}<D^{\prime \prime}$ and $b\left(D^{\prime \prime}\right)=b\left(D^{\prime}\right)-e\left(J_{+}^{\prime}\right) / 2+e\left(J_{-}^{\prime}\right) / 2$. Thus $D^{\prime \prime}$ is in $\bar{D}\left(F_{s}\right)$ and $\bar{D}\left(F_{\eta}\right)<\bar{D}\left(F_{s}\right)$. Moreover, it is not hard to see that by replacing $F$ by an element of $W(S)$ whose image in $W(\eta)$ is in the $\operatorname{Pic}^{0}(X \times \eta)$-orbit of $F_{\eta}$, we can get arbitrary disjoint subsets $J_{+}^{\prime}$ and $J_{-}^{\prime}$ of $J\left(F_{\eta}\right)$ in this way. Thus by Theorem 10.5 (1), we are done.

Definition. Let $F$ and $F^{\prime}$ be line bundles in $\operatorname{LB}_{\phi}^{0}(X) . F^{\prime}$ is said to be in the $\phi$-closure of $F$ if the following conditions are satisfied:

(i) There exist Delony polyhedra $D \in \bar{D}(F)$ and $D^{\prime} \in \bar{D}\left(F^{\prime}\right)$ with $D<D^{\prime}$ and $\rho($ rel.int $D) \subset \rho\left(\right.$ rel.int $\left.D^{\prime}\right)$. Let $D^{\prime}$ be of the form

$$
D^{\prime}=D+\left\{\sum_{j \in J_{+}^{\prime}} t_{j}\left(-e_{j}\right)+\sum_{j \in J_{-}^{\prime}} t_{j} e_{j} ; 0 \leqslant t_{j} \leqslant 1\right\}
$$

for a decomposition Supp $D^{\prime}=\operatorname{Supp} D \amalg J_{+}^{\prime} \amalg J_{-}^{\prime}$. Then

(ii) $F^{\prime}$ is $\theta_{X}$-isomorphic to the kernel of the composition 


$$
\begin{aligned}
& \mathfrak{L}(F)\left(-\sum_{j \in J_{+}^{\prime}}\left[Q_{j}^{+}\right]-\sum_{j \in J_{-}^{-}}\left[Q_{j}^{-}\right]\right) \hookrightarrow \mathfrak{L}(F) \rightarrow \mathfrak{L}(F) / F \\
& \rightarrow \underset{j \in J-\text { Supp } D}{\bigoplus_{j}} k\left(Q_{j}\right) \otimes_{\theta_{x}} \mathcal{L}(F) / F
\end{aligned}
$$

of the canonical inciusion and the canonical projections.

Proposition 12.11. Let $q$ and $q^{\prime}$ be closed points of $R_{\phi}(E / P)$ defined in $\S 11$. Then $q^{\prime}$ is in the closure of the $\mathrm{GL}(E)$-orbit of $q$ if and only if $G_{q^{\prime}}(-\tilde{n})$ is in the $\phi$-closure of $G_{q}(-\tilde{n})$ as elements of $\mathrm{LB}_{\phi}^{0}(X)$.

Proof. By the choice of $\tilde{n}$ in the definition of $R_{\phi}(E / P)$, a point $q^{\prime \prime}$ is in the $\mathrm{GL}(E)$-orbit of $q$ if and only if $G_{q^{\prime \prime}} \cong G_{q}$. Thus $q^{\prime}$ is in the closure of the $\mathrm{GL}(E)$-orbit of $q$ if and only if there exist the spectrum $S=\{\eta, s\}$ of a discrete valuation ring with $k(s)=k$ and a flat family of line bundles $\tilde{G} \in W(S)$ such that $\tilde{G}_{\eta} \cong k(\eta) \otimes_{k} G_{q}$ and $\tilde{G}_{s} \cong G_{q^{\prime}}$ and that there is a surjective homomorphism $\theta_{X \times S} \otimes_{k} E \rightarrow \tilde{G}$. Note that this last condition is superfluous, since other conditions imply that $R^{1} P_{2_{*}} \tilde{G}=0$ hence $P_{2_{*}} \tilde{G}$ is a free $R$-module of rank $P(0)$, which is noncanonically isomorphic to $R^{*} \otimes_{k} E$. Let $\tilde{F}=\tilde{G} \otimes P_{1}^{*} \theta_{X}(-\tilde{n}), F=G_{q}(-\tilde{n})$ and $F^{\prime}=G_{q^{\prime}}(-\tilde{n})$. Then we should have $\tilde{F}_{\eta} \cong k(\eta) \otimes_{k} F$ and $\tilde{F}_{s} \cong F^{\prime}$. Let $a: \mathcal{L}(F) \rightarrow \mathcal{L}(F) / F \cong \bigoplus_{j \in J(F)} k\left(Q_{j}\right)$ be the canonical projection. Then $a$ is a presentation over $k$ with $\operatorname{ker}(a)=F$. Its base extension $a^{*}$ to $R$

$$
a^{*}: R \otimes_{k} \mathcal{L}(F) \rightarrow R \otimes_{k} \mathcal{L}(F) / R \otimes_{k} F \cong \bigoplus_{j \in J(F)} R\left(Q_{j}\right)
$$

is a presentation over $S$ with $\operatorname{ker}\left(a^{*}\right)=R \otimes_{k} F$. Since both $R \otimes_{k} F$ and $\tilde{F}$ have the same image $k(\eta) \otimes_{k} F$ in $W(\eta)$, we see by Proposition 12.7 that $\tilde{F}$ is obtained as the kernel of the restriction $a^{\prime}$ to $R \otimes_{k} E(F)$ of $\left(a^{*}\right)_{\eta} \circ b$ for an element $b$ in $C_{0}\left(\Gamma(X), k(\eta)^{*}\right)$ acting by the multiplication of $b_{i}$ on the restriction of $k(\eta) \otimes_{k} \mathcal{L}(F)$ to $\tilde{X}_{i}$. Thus we have an exact sequence

$$
0 \rightarrow \tilde{F} \rightarrow R \otimes_{k} \mathcal{L}(F) \stackrel{a^{\prime}}{\rightarrow} \underset{j \in J(F)}{\bigoplus_{j}} R\left(Q_{j}\right) \rightarrow 0 .
$$

The restriction $a_{s}^{\prime}$ of $a^{\prime}$ to $X \times s$ is a presentation over $k$ with $\operatorname{ker}\left(a_{s}^{\prime}\right)=\tilde{F}_{s}$ but need not be strict. Let $\beta=$ ord $b \in C_{0}(\Gamma(X), Z)$ and let

$$
\begin{gathered}
J_{+}^{\prime}=\left\{j \in J(F) ;\left(\delta \beta, e_{j}\right)>0\right\}, \\
J_{-}^{\prime}=\left\{j \in J(F) ;\left(\delta \beta, e_{j}\right)<0\right\}, \\
J_{0}^{\prime}=\left\{j \in J(F) ;\left(\delta \beta, e_{j}\right)=0\right\} .
\end{gathered}
$$

Then we see easily that

$$
\mathfrak{L}\left(\tilde{F}_{s}\right)=\mathfrak{L}(F)\left(-\sum_{j \in J_{+}^{\prime}}\left[Q_{j}^{+}\right]-\sum_{j \in J_{-}^{\prime}}\left[Q_{j}^{-}\right]\right)
$$

and that $\tilde{F}_{s}$ is the kernel of the strict presentation over $k$ obtained by 
composing the canonical injection $\mathcal{L}\left(\tilde{F}_{s}\right) \rightarrow \mathcal{L}(F)$, the canonical projection $a$ : $\mathcal{L}(F) \rightarrow \mathfrak{L}(F) / F \cong \bigoplus_{j \in J(F)} k\left(Q_{j}\right)$ and the canonical projection $\bigoplus_{j \in J(F)} k\left(Q_{j}\right) \rightarrow \bigoplus_{j \in J_{0}^{\prime}} k\left(Q_{j}\right)$. As in the proof of Proposition 12.10, we see that for $D$ in $\bar{D}(F)$, the Delony polyhedron defined by

$$
D^{\prime}=D+\left\{\sum_{j \in J_{+}^{\prime}} t_{j}\left(-e_{j}\right)+\sum_{j \in J_{-}^{\prime}} t_{j} e_{j} ; 0 \leqslant t_{j} \leqslant 1\right\}
$$

is in $\bar{D}\left(\tilde{F}_{s}\right)$. Thus the rest of the proof is an immediate consequence of the following:

Lemma 12.12. Let $D<D^{\prime}$ be in $K(\Gamma)$. Then

$$
\rho(\text { rel.int } D) \subset \rho\left(\text { rel.int } D^{\prime}\right)
$$

if and only if there exists $\delta \beta \in \delta C_{0}(\Gamma, Z)$ such that

$$
D^{\prime}=D+\left\{\sum_{j \in J_{+}^{\prime}} t_{j}\left(-e_{j}\right)+\sum_{j \in J_{-}^{\prime}} t_{j} e_{j} ; 0 \leqslant t_{j} \leqslant 1\right\}
$$

where

$$
\begin{aligned}
& J_{+}^{\prime}=\left\{j \in J-\operatorname{Supp} D ;\left(\delta \beta, e_{j}\right)>0\right\}, \\
& J_{-}^{\prime}=\left\{j \in J-\operatorname{Supp} D ;\left(\delta \beta, e_{j}\right)<0\right\} .
\end{aligned}
$$

Proof. We first prove the "if" part. Suppose there exists $\delta \beta$ as in the lemma. There exists $c$ in $C_{1}(\Gamma, Z)$ such that $D=c+\left\{\Sigma_{j \in \operatorname{Supp} D} t_{j} e_{j} ; 0 \leqslant t_{j} \leqslant\right.$ $1\}$. Thus $D^{\prime}=c+\left\{\Sigma_{j \in J} t_{j} \varepsilon_{j} e_{j} ; 0 \leqslant t_{j} \leqslant 1\right\}$, where

$$
\varepsilon_{j}= \begin{cases}1 & \text { if } j \in \operatorname{Supp} D \amalg J_{-}^{\prime}, \\ -1 & \text { if } j \in J_{+}^{\prime}, \\ 0 & \text { otherwise. }\end{cases}
$$

Let $y$ be in rel.int $D$, i.e. $0<\left(y-c, e_{j}\right)<1$ for $j \in \operatorname{Supp} D$ and $\left(y-c, e_{j}\right)$ $=0$ for $j \notin \operatorname{Supp} D$. If we choose sufficiently small positive number $r$, then $y+r \delta \beta$ is in rel.int $D^{\prime}$. Indeed, $\left(y+r \delta \beta-c, e_{j}\right)=\left(y-c, e_{j}\right)+r\left(\delta \beta, e_{j}\right)$ is strictly between 0 and $\varepsilon_{j}$ for $j \in \operatorname{Supp} D \amalg J_{+}^{\prime} \amalg J_{-}^{\prime}$ and equals 0 otherwise. Thus $\rho(y)=\rho(y+r \delta \beta) \in \rho\left(\right.$ rel.int $\left.D^{\prime}\right)$. We next prove the "only if" part. As before, let

$$
\begin{aligned}
& D=c+\left\{\sum_{j \in \operatorname{Supp} D} t_{j} e_{j} ; 0 \leqslant t_{j} \leqslant 1\right\}, \\
& D^{\prime}=c+\left\{\sum_{j \in J} t_{j} \varepsilon_{j} e_{j} ; 0 \leqslant t_{j} \leqslant 1\right\},
\end{aligned}
$$

where $\varepsilon_{j}=1,-1$ or 0 , according as $j$ is in Supp $D \amalg J_{-}^{\prime}$, in $J_{+}^{\prime}$ or otherwise. Let $y$ be in rel.int $D$. Since $\rho(y)$ is in $\rho\left(\right.$ rel.int $\left.D^{\prime}\right)$, by assumption, there exists $\delta \beta^{\prime}$ in $\delta C_{0}(\Gamma, \mathbf{R})$ such that $y+\delta \beta^{\prime} \in$ rel.int $D^{\prime}$, i.e. $\left(y-c+\delta \beta^{\prime}, e_{j}\right)$ is strictly between 0 and $\varepsilon_{j}$ if $j \in \operatorname{Supp} D \amalg J_{+}^{\prime} \amalg J_{-}^{\prime}$ and equals 0 otherwise. But 
since $\left(y-c, e_{j}\right)=0$ if $j \notin \operatorname{Supp} D$, we see that $\left(\delta \beta^{\prime}, e_{j}\right)$ is strictly between 0 and $\varepsilon_{j}$ if $j \in J_{+}^{\prime} \amalg J_{-}^{\prime}$ and equals 0 if $j \notin \operatorname{Supp} D \amalg J_{+}^{\prime} \amalg J_{-}^{\prime}$. Consider the set of coboundaries $\delta \beta \in \delta C_{0}(\Gamma, \mathbf{R})$ with $\left(\delta \beta, e_{j}\right)$ positive, negative or 0 , according as $j \in J_{+}^{\prime}, j \in J_{-}^{\prime}$ or $j \notin \operatorname{Supp} D \amalg J_{+}^{\prime} \amalg J_{-}^{\prime}$. This set is not empty, since $\delta \beta^{\prime}$ belongs to it. Then there certainly exists a rational element $\delta \beta \in$ $\delta C_{0}(\Gamma, \mathbf{Q})$ in this set. Multiplying $\delta \beta$ by a suitable positive integer, we conclude that there exists an integral element $\delta \beta \in \delta C_{0}(\Gamma, Z)$ in this set, and we are done.

Definition. Line bundles $F_{1}$ and $F_{2}$ in $\operatorname{LB}_{\phi}^{0}(X)$ are said to be $\phi$-equivalent if there exists $F_{3}$ in $\mathrm{LB}_{\phi}^{0}(X)$ which is in the $\phi$-closure of both $F_{1}$ and $F_{2}$.

From this definition, we immediately see the following:

COROLlaRY 12.13. Let $q$ and $q^{\prime}$ be closed points of $R_{\phi}(E / P)$. Then the closures of their $\mathrm{GL}(E)$-orbits intersect if and only if $G_{q}(-\tilde{n})$ and $G_{q}(-\tilde{n})$ are $\phi$-equivalent as elements of $\mathrm{LB}_{\phi}^{0}(X)$.

We are now ready to state the main theorem of this section.

THEOREM 12.14. Let $\phi$ be in $\partial C_{1}(\Gamma(X), \mathbf{R})$. Then the reduced projective algebraic scheme $\mathrm{Jac}_{\phi}(X)$ and its open subscheme $\mathrm{Jac}_{\phi-s t a b l e}(X)$ admit an action of the generalized Jacobian variety $\mathrm{Pic}_{X}^{0}$ and satisfy the following properties: there is a Pic $_{X}^{0}$-admissible morphism of set functors

$$
w_{\phi}: W_{\phi} \rightarrow \operatorname{Jac}_{\phi}(X)
$$

(i) which induces an isomorphism of functors

$$
w_{\phi-s t a b l e}: W_{\phi-s t a b l e} \rightarrow \mathrm{Jac}_{\phi-\text { stable }}(X)
$$

i.e. $W_{\phi-s t a b l e}$ is represented by $\mathrm{Jac}_{\phi-s t a b l e}(X)$.

(ii) The map

$$
w_{\phi}(\operatorname{Spec} k): \operatorname{LB}_{\phi}^{0}(X)=W_{\phi}(\operatorname{Spec} k) \rightarrow \operatorname{Jac}_{\phi}(X)(\operatorname{Spec} k)
$$

is surjective and induces a bijection from the set of $\phi$-equivalence classes in $\mathrm{LB}_{\phi}^{0}(X)$ to $\mathrm{Jac}_{\phi}(X)(\operatorname{Spec} k)$.

(iii) $w_{\phi}$ is universal among morphisms from $W_{\phi}$ to representable functors, i.e. if $w^{\prime}: W_{\phi} \rightarrow Y$ is a morphism of set functors with $Y$ representable, then there is a unique morphism of schemes $u$ : $\operatorname{Jac}_{\phi}(X) \rightarrow Y$ such that $u \circ w_{\phi}=w^{\prime}$.

COROLLARY 12.15. $\operatorname{Jac}_{\phi}(X)$ represents the functor $W_{\phi}$ if and only if $\phi$ is nondegenerate, i.e. $\phi-\partial e(J) / 2$ is in the interior of a top-dimensional polyhedron in $\operatorname{Arr}(\Gamma(X))$.

Proof OF THEOREM 12.14. It is now standard in the geometric invariant theory to deduce this theorem from Theorem 11.4.

From what we saw in $\$ 11$, the universal quotient sheaf $G$ on $X \times$ $R_{\phi}(E / P)$ satisfies the property that $G_{q}(-\tilde{n}) \in \mathrm{LB}_{\phi}^{0}(X)$ for all closed points $q$ 
of $R_{\phi}(E / P)$. Thus $P_{1}{ }^{*} \theta_{X}(-\tilde{n}) \otimes G$ determines an element of $W_{\phi}\left(R_{\phi}(E / P)\right)$ by the definition of $W_{\phi}$ at the beginning of $\S 12$. Thus we have a morphism of functors

$$
u: R_{\phi}(E / P) \rightarrow W_{\phi} .
$$

It assigns to a closed point $q \in R_{\phi}(E / P)$ the element $G_{q}(-\tilde{n}) \in$ $W_{\phi}(\operatorname{Spec} k) . u$ is obviously invariant under the action of $\mathrm{GL}(E)$ on $R_{\phi}(E / P)$, since $q$ and $q^{\prime}$ are in the same $\mathrm{GL}(E)$-orbit if and only if $G_{q} \cong G_{q^{\prime}}$ by $\$ 11$.

Let $S$ be a $k$-scheme and let $F \in W_{\phi}^{*}(S)$ be a flat family of line bundles on $X$ parametrized by $S$, which defines an element of $W_{\phi}(S)$. Then by the choice of $\tilde{n}$, we see that $R^{1} P_{2_{*}}\left(F \otimes P_{1}^{*} \theta_{X}(\tilde{n})\right)=0, P_{2_{*}}\left(F \otimes P_{1}^{*} \theta_{X}(\tilde{n})\right)$ is a locally free sheaf on $S$ of rank $P(0)$ and the canonical homomorphism

$$
P_{2}^{*} P_{2_{*}}\left(F \otimes P_{1}^{*} \vartheta_{X}(\tilde{n})\right) \rightarrow F \otimes P_{1}^{*} \vartheta_{X}(\tilde{n})
$$

is surjective. There exists an open covering $S=\cup_{v} S_{v}$ such that the restriction of $P_{2_{*}}\left(F \otimes P_{1}^{*} \theta_{X}(\tilde{n})\right)$ to $S_{\nu}$ is free. Then there exists a surjective homomorphism from $\theta_{X \times S}, \otimes_{k} E$ to the restriction of $F \otimes P_{1}^{*} \theta_{X}(\tilde{n})$ to $X \times S_{\nu}$. Thus by the universality of $R_{\phi}(E / P)$, there exist morphisms $S_{\nu} \rightarrow$ $R_{\phi}(E / P)$, determined up to $\mathrm{GL}(E)$-action, which induces the restriction of $F \otimes P_{1}^{*} \Theta_{X}(\tilde{n})$ on $X \times S_{v}$ by pull-back. Composing these morphisms with the projection $R_{\phi}(E / P) \rightarrow \mathrm{Jac}_{\phi}(X)$, we have morphisms $S_{\nu} \rightarrow \mathrm{Jac}_{\phi}(X)$. By the $\mathrm{GL}(E)$-invariance of the quotient, they patch up to be a morphism $S \rightarrow$ $\mathrm{Jac}_{\phi}(X)$, which is uniquely determined by the image of $F \in W_{\phi}^{*}(S)$ in $W_{\phi}(S)=W_{\phi}^{*}(S) / \operatorname{Pic}(S)$. We thus get a map

$$
W_{\phi}(S) \rightarrow \mathrm{Jac}_{\phi}(X)(S),
$$

which is obviously functorial in $S$. Thus we obtain a morphism of functors

$$
w_{\phi}: W_{\phi} \rightarrow \operatorname{Jac}_{\phi}(X)
$$

which obviously induces

$$
w_{\phi \text {-stable }}: W_{\phi \text {-stable }} \rightarrow \mathrm{Jac}_{\phi \text {-stable }}(X)
$$

$w_{\phi}$ is universal among morphisms from $W_{\phi}$ to representable functors. Indeed, if $w^{\prime}: W_{\phi} \rightarrow Y$ is a morphism to a representable functor, we have a morphism $w^{\prime} \circ u: R_{\phi}(E / P) \rightarrow Y$, which is obviously $\mathrm{GL}(E)$-invariant. Since $\mathrm{Jac}_{\phi}(X)$ is a good quotient, hence a categorical quotient, by Theorem 11.4, $w^{\prime} \circ u$ factors through a morphism $\mathrm{Jac}_{\phi}(X) \rightarrow Y$. Since closed points $q$ and $q^{\prime}$ of $R_{\phi}(E / P)$ go into the same point of $\operatorname{Jac}_{\phi}(X)$ if and only if the closures of their $\mathrm{GL}(E)$-orbits intersect by Theorem $11.4(1)$, we see that

$$
w_{\phi}(\operatorname{Spec} k): W_{\phi}(\operatorname{Spec} k)=\operatorname{LB}_{\phi}^{0}(X) \rightarrow \operatorname{Jac}_{\phi}(X)(\operatorname{Spec} k)
$$

induces a bijection from the set of $\phi$-equivalence classes in $\operatorname{LB}_{\phi}^{0}(X)$ to $\mathrm{Jac}_{\phi}(X)(\operatorname{Spec} k)$ by Corollary 12.13 . Since a modification $G^{\prime}$ by an invertible 
sheaf on $R_{\phi-\text { stable }}(E / P)$ of the restriction of the universal quotient sheaf $G$ to $X \times R_{\phi \text {-stable }}(E / P)$ descends to $X \times \mathrm{Jac}_{\phi \text {-stable }}(X)$ by Theorem 11.4 (5), we see that $G^{\prime} \otimes P_{1}^{*} \Theta_{X}(-\tilde{n})$ also descends to $X \times \mathrm{Jac}_{\phi-\text {-stable }}(X)$. Since $R_{\phi \text {-stable }}(E / P) \rightarrow \mathrm{Jac}_{\phi \text {-stable }}(X)$ is a geometric quotient, we conclude that $w_{\phi \text {-stable }}$ is an isomorphism. The statement about the Pic $_{X}^{0}$-action is straightforward. Corollary 12.15 is an immediate consequence of this theorem and Proposition 11.3.

Recall that at the beginning of $\S 6$ we introduced, for a graph $\Gamma$, a surjective map

$$
\mu: K_{\phi}(\Gamma) \rightarrow K_{\phi}^{0}(\Gamma),
$$

which defines an equivalence relation in $K_{\phi}(T): D_{1}$ and $D_{2}$ are $\phi$-equivalent when $\mu\left(D_{1}\right)=\mu\left(D_{2}\right)$. Given $D$ in $K_{\phi}(T), \mu(D)$ is the largest element $\phi$ equivalent to $D . \mu(D)$ is also characterized as the unique element in $K_{\phi}^{0}(\Gamma)$ which is $\phi$-eqivalent to $D$. Moreover, $D$ is $\phi$-stable if and only if $D$ is the only element in the $\phi$-equivalence class of $D$.

Since the projection $\rho: C_{1}(T, \mathbf{R}) \rightarrow H^{1}(\Gamma, \mathbf{R})$ induces a bijection

$$
\rho: K_{\phi}^{0}(\Gamma) \stackrel{\sim}{\rightarrow} \operatorname{Del}_{\phi}\left(H^{1}(\Gamma, \mathbf{R})\right)
$$

and since the right-hand side is a polyhedral decomposition of $H^{1}(\Gamma, \mathbf{R})$, we have the following, whose proof is left to the reader:

LemaA 12.16. Let $D<D^{\prime}$ be $\phi$-semistable Delony polyhedra in $K_{\phi}(T)$. Then $D$ and $D^{\prime}$ are $\phi$-equivalent if and only if

$$
\rho(\text { rel.int } D) \subset \rho\left(\text { rel.int } D^{\prime}\right) \text {. }
$$

Taking the quotient of

$$
\begin{aligned}
& K_{\phi}(\Gamma) \stackrel{\mu}{\rightarrow} K_{\phi}^{0}(\Gamma) \stackrel{\rho}{\rightarrow} \operatorname{Del}_{\phi}\left(H^{1}(\Gamma, \mathbf{R})\right) \\
& \underset{K_{\phi}^{0}(\Gamma)}{ }
\end{aligned}
$$

with respect to the translation action of $H_{1}(\Gamma, \mathrm{Z})$, we obtain

$$
\begin{aligned}
& \bar{K}_{\phi}(\Gamma) \stackrel{\bar{\mu}}{\rightarrow} \bar{K}_{\phi}^{0}(\Gamma) \stackrel{\bar{\rho}}{\rightarrow} \overline{\operatorname{Del}}_{\phi}\left(H^{1}(\Gamma, \mathbf{R})\right) \\
& \bar{K}_{\phi}^{0}(\Gamma)
\end{aligned}
$$

where the last term is defined as follows:

Definition. The Namikawa cell complex

$$
\overline{\operatorname{Del}}_{\phi}\left(H^{1}(\Gamma, \mathbf{R})\right)=\operatorname{Del}_{\phi}\left(H^{1}(\Gamma, \mathbf{R})\right) / \rho\left(H_{1}(\Gamma, \mathbf{Z})\right)
$$

is the finite cell complex obtained as the quotient of the Namikawa 
decomposition $\operatorname{Del}_{\phi}\left(H^{1}(\Gamma, \mathbf{R})\right)$ with respect to the translation action of $\rho\left(H_{1}(\Gamma, \mathbf{Z})\right)$. We call a cell in this complex a Namikawa cell. For a Namikawa cell $\bar{N}$ in $\overline{\operatorname{Del}}_{\phi}\left(H^{1}(T, \mathbf{R})\right)$, we define $\operatorname{dim} \bar{N}, \operatorname{Supp} \bar{N}$ and $\partial b(\bar{N})$ to be $\operatorname{dim} D$, Supp $D$ and $\partial b(D)$, respectively, for $D$ in $K_{\phi}^{0}(\Gamma)$ with $\rho(D) \in \bar{N}$, determined up to the translation by elements of $H_{1}(\Gamma, Z)$.

Definition. $\bar{D}_{1}$ and $\bar{D}_{2}$ in $\bar{K}_{\phi}(\Gamma)$ are said to be $\phi$-equivalent if $\bar{\mu}\left(\bar{D}_{1}\right)=$ $\bar{\mu}\left(\bar{D}_{2}\right)$, i.e. there exist $D_{1} \in \bar{D}_{1}$ and $D_{2} \in \bar{D}_{2}$ such that $D_{1}$ and $D_{2}$ are $\phi$-equivalent.

Applying Lemma 12.16 to $\Gamma=\Gamma(X)$, we see by Corollary 12.13 that the $\phi$-equivalence of $F_{1}$ and $F_{2}$ in $\operatorname{LB}_{\phi}^{0}(X)$ implies the $\phi$-equivalence of $\bar{D}\left(F_{1}\right)$ and $\bar{D}\left(F_{2}\right)$. By Proposition 12.11, we conclude that in a $\phi$-equivalence class of line bundles in $\mathrm{LB}_{\phi}^{0}(X)$ there is a unique $F$ with $\bar{D}(F) \in K_{\phi}^{0}(T(X))$, corresponding to the unique closed $\mathrm{GL}(E)$-orbit in the equivalence class of points defined by the good quotient $R_{\phi}(E / P) \rightarrow \mathrm{Jac}_{\phi}(X)$.

By Theorems 10.5 and 12.4 and Proposition 12.3, we can relate the notions of $\phi$-equivalence in $\mathrm{LB}_{\phi}^{0}(X)$ and $\bar{K}_{\phi}(\Gamma(X))$ more precisely as follows:

Proposition 12.17. The map $\bar{D}$ of Theorem 10.5 induces bijections

$$
\begin{array}{ll}
W_{\phi} / \mathrm{Pic}_{X}^{0} & \stackrel{\rightarrow}{K_{\phi}}(\Gamma(X)), \\
\mathrm{Jac}_{\phi}(X) / \operatorname{Pic}_{X}^{0} & \stackrel{\rightarrow}{\operatorname{Del}_{\phi}}\left(H^{1}(\Gamma(X), \mathbf{R})\right)
\end{array}
$$

compatible with the map induced by $w_{\phi}$ for the first column and $\bar{\rho} \circ \bar{\mu}$ for the second column. For a Namikawa cell $\bar{N}$ in $\overline{\operatorname{Del}}_{\phi}\left(H^{1}(\Gamma(X), \mathbf{R})\right)$, let $\mathbf{O}(\bar{N})$ be the corresponding $\mathrm{Pic}_{X}^{0}$-orbit in $\mathrm{Jac}_{\phi}(X)$. Then we have:

(i) The map $\mathbf{O}$ is order reversing, i.e. $\bar{N}$ is a face of $\bar{N}^{\prime}$, if and only if $\mathbf{O}\left(\bar{N}^{\prime}\right)$ is in the closure of $\mathbf{O}(\bar{N})$.

(ii) $\mathbf{O}(\bar{N})$ is canonically isomorphic to $\left.\operatorname{Pic}_{X(J-\operatorname{Supp}}^{m} \bar{N}\right)$ where $m=\partial b(\bar{N})-$ $d(\operatorname{Supp} \bar{N}) / 2$. Especially

(iii) $\operatorname{dim} \bar{N}$ is equal to the codimension of $\mathbf{O}(\bar{N})$ in $\mathrm{Jac}_{\phi}(X)$.

In the definition immediately after Proposition 7.6, we denoted by $B_{r}(\Gamma(X) ; \phi)$ the number of $r$-dimensional Namikawa cells in $\overline{\operatorname{Del}}_{\phi}\left(H^{1}(\Gamma(X), \mathbf{R})\right)$ for $0 \leqslant r \leqslant h(T(X))=h$. For each 0-dimensional Namikawa cell $\bar{\xi}, \mathbf{O}(\bar{\xi})=$ Pic $_{X}^{\partial \bar{\xi}}$ is noncanonically isomorphic to the generalized Jacobian variety $\operatorname{Pic}_{X}^{0}$. Thus we conclude that $\mathrm{Jac}_{\phi}(X)$ is a compactification of the union of $B_{0}(\Gamma(X) ; \phi)$ copies of $\mathrm{Pic}_{X}^{0}$. This compactification $\mathrm{Jac}_{\phi}(X)$ depends only on the polyhedron in $\operatorname{Arr}(\Gamma(X))$ whose relative interior contains $\phi-\partial e(J) / 2$ by Proposition 11.2 (i). In particular, when $\phi$ is nondegenerate,

$$
B_{r}(\Gamma(X) ; \phi)=\left(\begin{array}{l}
h \\
r
\end{array}\right) \cdot \operatorname{complexity}(\Gamma(X))
$$

is independent of $\phi$, by Theorem 7.7. Thus by Proposition 12.17, the number 
of $\mathrm{Pic}_{X}^{0}$-orbits of each codimension is independent of $\phi$. However, the actual incidence relation among these orbits might depend on $\phi$ (cf. §8, Example (3) (ii)).

13. The structure of the compactifications $\operatorname{Jac}_{\phi}(X)$. Let $H \supset H^{\prime}$ be a free Z-module of rank $h$ and a submodule of finite index. Consider a polyhedral decomposition Dec of $H \otimes_{\mathrm{Z}} \mathrm{R}$ by bounded convex polyhedra such that

(i) the set of vertices $\mathrm{Sk}^{0}(\mathrm{Dec})$ is contained in $H$, and

(ii) Dec is invariant under the translation action of $H^{\prime}$.

We denote by $\overline{\mathrm{Dec}}=\mathrm{Dec} / H^{\prime}$ the quotient cell complex, which is a cell decomposition of the real torus $H \otimes_{\mathrm{Z}} \mathbf{R} / H^{\prime} \cong \mathbf{R}^{h} / \mathbf{Z}^{h}$.

For polyhedral cones and torus embeddings, we refer the reader to Mumford et al. [24] or Miyake-Oda [17].

For $\xi \in \mathrm{Sk}^{0}(\mathrm{Dec})$, consider the finite rational polyhedral decomposition (f.r.p. decomposition for short) of $H \otimes_{\mathbf{Z}} \mathbf{R}$

$$
\Delta(\xi)=\{\sigma(\xi, N) ; \xi \in N \in \operatorname{Dec}\}
$$

consisting of polyhedral cones $\sigma(\xi, N)$ in $H \otimes_{\mathrm{Z}} \mathbf{R}$ with the vertex at the origin generated by the set $\{x-\xi ; x \in N\}$, i.e. if $\xi, \xi_{1}, \ldots, \xi_{\text {r }}$ are the vertices of the polyhedron $N$, then

$$
\sigma(\xi, N)=\mathbf{R}_{>0}\left(\xi_{1}-\xi\right)+\mathbf{R}_{>0}\left(\xi_{2}-\xi\right)+\cdots+\mathbf{R}_{>0}\left(\xi_{r}-\xi\right) .
$$

Let us denote $T=G_{m} \otimes H$. Then by the general theory, we obtain a torus embedding $T \subset \operatorname{Temb}(\xi)$ corresponding to the f.r.p. decomposition $\Delta(\xi)$. It is clear that $\operatorname{Temb}(\xi)$ is a complete variety. There exists a bijection

$$
\text { orb }_{\xi}:\{N \in \operatorname{Dec} ; N \ni \xi\} \stackrel{\sim}{\rightarrow}\{T \text {-orbits in } \operatorname{Temb}(\xi)\}
$$

such that (1) $\operatorname{dim} N$ is the codimension of $\operatorname{orb}_{\xi}(N)$ in $\operatorname{Temb}(\xi)$ and (2) $\operatorname{orb}_{\xi}(N)$ is in the closure of $\operatorname{orb}_{\xi}\left(N^{\prime}\right)$ if and only if $N^{\prime}$ is a face of $N$.

From now on, we let

$$
\begin{gathered}
H=H^{1}(\Gamma(X), \mathbf{Z}), \quad H^{\prime}=\rho\left(H_{1}(\Gamma(X), \mathbf{Z})\right), \\
\operatorname{Dec}=\operatorname{Del}_{\phi}\left(H^{1}(\Gamma(X), \mathbf{R})\right)
\end{gathered}
$$

for a curve $X$ and $\phi \in \partial C_{1}(T(X), \mathbf{R})$. They satisfy the requirements at the beginning of this section by Proposition 6.1.

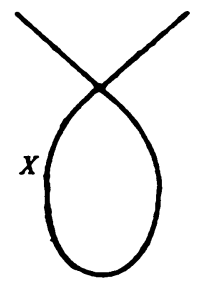

$\Gamma(X)$

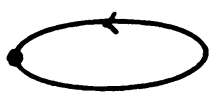

FIGURE 23 
EXAMPLES 13.1. (1) $X$ is irreducible with one double point, whose graph looks like Figure 23 (cf. §8, (1)). In this case, necessarily $\phi=0, H=H^{\prime}$ and Dec looks like Figure 24. Thus for any $\xi \in H, \Delta(\xi)$ looks like Figure 25, hence $\operatorname{Temb}(\xi)=\mathbf{P}_{1}$.
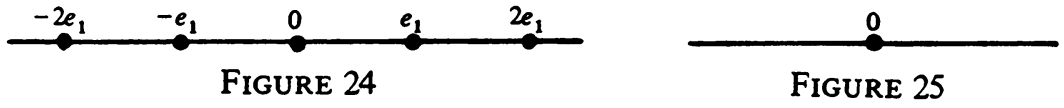

(2) $X$ is irreducible with two double points, whose graph looks like Figure 26 (cf. §8, (1)). Again we have $\phi=0$ and $H=H^{\prime}$. Thus Dec and $\Delta(\xi)$ for any $\xi \in H$ look like Figure 27. We have $\operatorname{Temb}(\xi)=\mathbf{P}_{1} \times \mathbf{P}_{1}$.
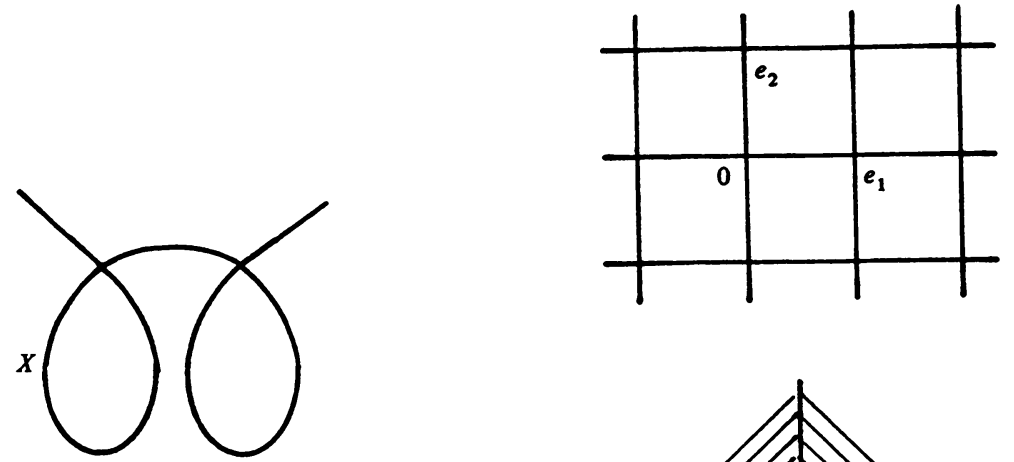

$\Gamma(X)$
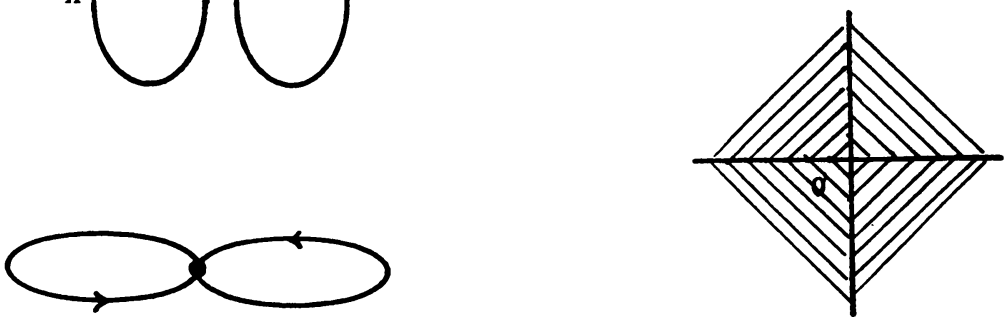

FIGURE 26

FIGURE 27

(3) $X$ has two components meeting at three points, whose graph looks like Figure 28 ( $\$ 8,(2 \mathrm{iii}))$.
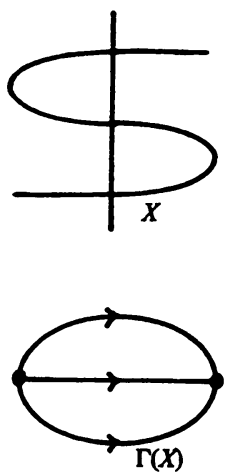

FigURE 28

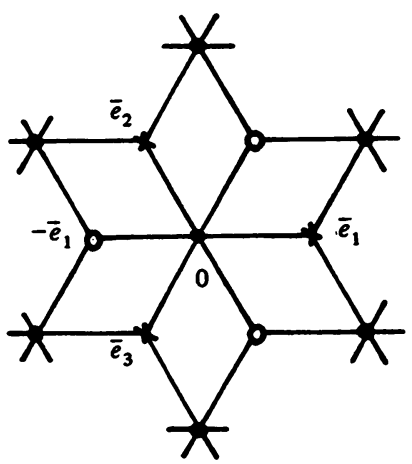

FIGURE 29 
(a) Let $\phi=0$ (cf. $\S 8,(2 i i i a)$ ). Then Dec looks like Figure 29. At the thick lattice points (e.g. $\xi=0$ ), the white lattice points (e.g. $\xi=-\bar{e}_{1}$ ) and the crossed lattice points (e.g. $\left.\xi=\bar{e}_{1}\right), \Delta(\xi)$ look respectively like Figure 30 . Since

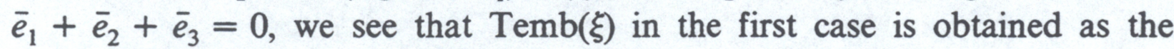
blowing up of $\mathbf{P}_{2}$ along the three coordinate vertices. In the other two cases, $\operatorname{Temb}(\xi)=\mathbf{P}_{2}$.
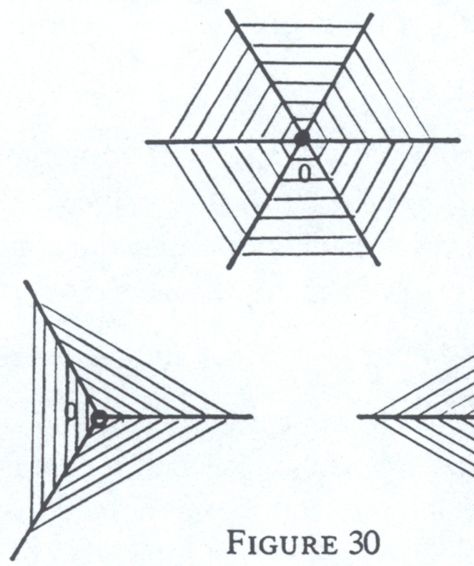

FIGURE 30

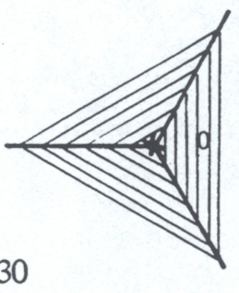

(b) If $\phi=\left(v_{1}-v_{2}\right) / 2$ (cf. §8, (2iiib)), then Dec looks like Figure 31. At the thick lattice points (e.g. $\xi=0)$ and the crossed lattice points (e.g. $\left.\xi=\bar{e}_{1}\right), \Delta(\xi)$ look respectively like Figure 32 . In both cases, we have $\operatorname{Temb}(\xi)=\mathbf{P}_{2}$. For $\xi \in \mathbf{S k}^{0}(\mathrm{Dec})$ consider the fiber bundle

$$
\operatorname{Pic}_{X}^{\partial \xi} \times{ }^{T} \operatorname{Temb}(\xi)
$$

associated with the principal $T$-bundle $\operatorname{Pic}_{X}^{\partial \xi}$ over $\operatorname{Pic}_{X}^{\partial \xi}$ and the $T$-action on $\operatorname{Temb}(\xi)$. Note that it depends only on the image $\bar{\xi}$ of $\xi$ in $\overline{\operatorname{Dec}}$. We thus denote it also by

$$
\operatorname{Pic}_{X}^{\partial \bar{\xi}} \times^{T} \operatorname{Temb}(\bar{\xi})
$$

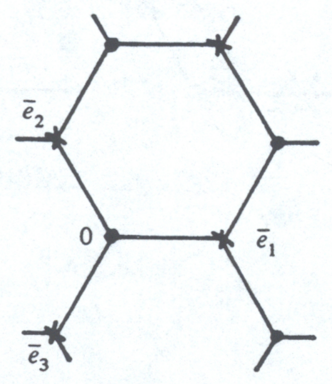

FIGURE 31

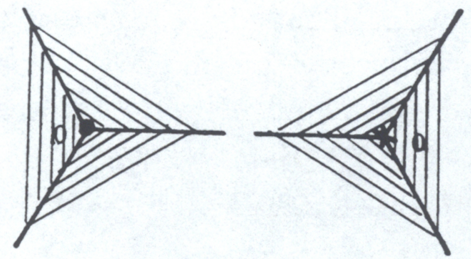

FIGURE 32 
THEOREM 13.2. Let $\phi$ be in $\partial C_{1}(\Gamma(X), \mathbf{R})$. Then for $\bar{\xi}$ in $\mathrm{Sk}^{0}\left(\overline{\operatorname{Del}}_{\phi}\left(H^{1}(\Gamma(X), \mathbf{R})\right)\right)$ there exists a morphism

$$
u_{\xi}: \operatorname{Pic}_{X}^{\partial \xi} \times^{T} \operatorname{Temb}(\bar{\xi}) \rightarrow \operatorname{Jac}_{\phi}(X) .
$$

The normalization of $\operatorname{Jac}_{\phi}(X)$ can be identified with the disjoint union of $\operatorname{Pic}_{X}^{\partial \bar{\xi}} \times^{T} \operatorname{Temb}(\bar{\xi})$ with $\bar{\xi}$ running over $\operatorname{Sk}^{0}\left(\overline{\operatorname{Del}}_{\phi}\left(H^{1}(\Gamma(X), \mathbf{R})\right)\right)$. For a pair $\xi \in N \in \operatorname{Del}_{\phi}\left(H^{1}(\Gamma(x), \mathbf{R})\right)$, there exists an isomorphism

$$
g_{\xi}(N): \operatorname{Pic}_{X(J-S u p p \bar{N})}^{m(\bar{N})} \stackrel{\sim}{\rightarrow} \operatorname{Pic}_{X}^{\partial \bar{\xi}} \times^{T} \operatorname{orb}_{i \xi}(\bar{N})
$$

with $m(\bar{N})=\partial b(\bar{N})-d(\operatorname{Supp} \bar{N}) / 2$ such that $u_{\bar{\xi}} \circ g_{\xi}(N)$ coincides with the isomorphism $\left.\operatorname{Pic}_{X(J-S u p p}^{m} \bar{N}\right) \stackrel{\sim}{\rightarrow} \mathbf{O}(\bar{N})$ of Proposition 12.17(ii). $\operatorname{Jac}_{\phi}(X)$ is obtained from the above disjoint union by the identification maps $g_{\xi}(N)$ with $N$ and $\xi$ running over all Namikawa polyhedra and their vertices.

COROLlaRy 13.3. If $\phi \in \partial C_{1}(\Gamma(X), \mathbf{R})$ is nondegenerate, then the normalization of $\mathrm{Jac}_{\phi}(X)$ is nonsingular.

Proof of 13.3. It is enough to show that $\operatorname{Temb}(\xi)$ is nonsingular for any $\xi$. Let $\xi \in N$ be a top-dimensional Namikawa polyhedron, and let $\tilde{N}$ be the unique element in $K_{\phi}^{0}$ with $\rho(\tilde{N})=N$. Then by Theorem 7.7(i), $J-\operatorname{Supp} \tilde{N}$ is the set of edges in a spanning tree of $\Gamma(X)$. Since $\tilde{N}$ is of the form

$$
\tilde{N}=\tilde{\xi}+\left\{\sum_{j \in \operatorname{Supp} \tilde{N}} t_{j} \varepsilon_{j} e_{j} ; 0<t_{j} \leqslant 1\right\}
$$

with $\varepsilon_{j}=1$ or -1 , we see that the cone $\sigma(\xi, N)$ is of the form

$$
\sigma(\xi, N)=\sum_{j \in \operatorname{Supp} N} \mathbf{R}_{>0} \varepsilon_{j} \rho\left(e_{j}\right) .
$$

As we saw immediately before Lemma $4.6,\left\{\rho\left(e_{j}\right) ; j \in \operatorname{Supp} N\right\}$ is a Z-basis of $H^{1}(\Gamma(X), \mathrm{Z})$. Thus we are done by the general theory of torus embeddings.

Proof of 13.2. In this proof, we let

$$
\begin{gathered}
C=C_{1}(\Gamma(X), \mathbf{Z}), \quad \tilde{T}=G_{m} \otimes C, \quad K=K(\Gamma(X)), \\
K_{\phi}=K_{\phi}(\Gamma(X)), \quad K_{\phi}^{0}=K_{\phi}^{0}(\Gamma(X)), \quad K_{\phi-\text { stable }}=K_{\phi-\text { stable }}(\Gamma(X)) .
\end{gathered}
$$

We saw in $\$ 6$ that $K_{\phi}$ consists of faces of $K_{\phi}^{0}, \mathrm{Sk}^{0}\left(K_{\phi-\text { stable }}\right)=\operatorname{Sk}^{0}\left(K_{\phi}^{0}\right)$ and that $\rho$ induces a bijection $K_{\phi}^{0} \rightarrow$ Dec. For $N$ in Dec, we denote by $\tilde{N}$ the unique Delony polyhedron in $K_{\phi}^{0}$ such that $\rho(\tilde{N})=N$. Thus by definition, we have Supp $N=\operatorname{Supp} \tilde{N}$ and $\partial b(N)=\partial b(\tilde{N})$. For $\tilde{\xi}$ in $\operatorname{Sk}^{0}\left(K_{\phi}^{g}\right)$, we define a finite rational partial polyedral decomposition (f.r.p.p. decomposition for short) of $C \otimes_{\mathbf{Z}} \mathbf{R}$ by

$$
\tilde{\Delta}(\tilde{\xi})=\left\{\tilde{\boldsymbol{\sigma}}(\tilde{\xi}, D) ; \tilde{\xi} \in D \in K_{\phi}\right\}
$$


where $\tilde{\sigma}(\tilde{\xi}, D)$ is the cone in $C \otimes_{\mathbf{Z}} \mathbf{R}$ with the vertex at the origin generated by $\{\tilde{x}-\tilde{\xi} ; \tilde{x} \in D\}$. As before, there exists a bijection

$$
\text { ỡ } b_{\xi}:\left\{D \in K_{\phi} ; D \ni \tilde{\xi}\right\} \stackrel{\sim}{\rightarrow}\{\tilde{T} \text {-orbits in } \tilde{\operatorname{T}} \operatorname{emb}(\tilde{\xi})\},
$$

where $\tilde{T} \subset \tilde{T} e m b(\tilde{\xi})$ is the torus embedding corresponding to $\tilde{\Delta}(\tilde{\xi})$. In this case, $\tilde{T} \operatorname{emb}(\tilde{\xi})$ need not be complete, but it is clear that $\tilde{\text { Temb }}(\tilde{\xi})$ is a $\tilde{T}$-stable open subset of the $\tilde{T}$-embedding $\Pi_{j \in J} \mathbf{P}_{1}$ corresponding to the f.r.p. decomposition $\{\tilde{\sigma}(\tilde{\xi}, D) ; D \in K\}$.

(i) By Corollary $12.4, \operatorname{StPres}\left(\partial \tilde{\xi}, J\right.$;?) is a principal $\tilde{T}$-bundle over $\operatorname{Pic}_{\tilde{X}}^{\partial \tilde{\xi}}$ and Pic $_{X}^{\partial \tilde{\xi}}$ is its quotient by the kernel $G_{m} \otimes \delta C_{0}(\Gamma(X), Z)$ of the canonical surjective homomorphism $\tilde{T} \rightarrow T$.

(ii) By Proposition 12.1, it is clear that

$$
\operatorname{Pres}(\partial \tilde{\xi}, J ; ?)=\operatorname{StPres}(\partial \tilde{\xi}, J ; ?) \times^{\dot{T}} \prod_{j \in J} \mathbf{P}_{1},
$$

the fiber bundle over $\operatorname{Pic}_{X}^{\partial \tilde{\xi}}$ associated with $\operatorname{StPres}(\partial \tilde{\xi}, J ;$ ?) and the $\tilde{T}$-action on $\Pi_{j \in J} \mathbf{P}_{1}$.

(iii) Let us consider its open subset

$$
\operatorname{StPres}(\partial \tilde{\xi}, J ; ?) \times \tilde{T} \tilde{\operatorname{Temb}}(\tilde{\xi}) \text {. }
$$

The kernel of the restriction to this open set of the universal presentation over $\operatorname{Pres}(\partial \tilde{\xi}, J ;$ ?) defines a morphism

$$
\tilde{v}_{\xi}: \operatorname{StPres}(\partial \tilde{\xi}, J ; ?) \times^{\tilde{T}} \tilde{\operatorname{T}} \operatorname{emb}(\tilde{\xi}) \rightarrow W_{\phi} .
$$

Indeed, let $\tilde{\xi} \in D \in K_{\phi}$ with

$$
D=\tilde{\xi}+\left\{\sum_{j \in J_{+}^{\prime}} t_{j}\left(-e_{j}\right)+\sum_{j \in J_{-}^{\prime}} t_{j} e_{j} ; 0 \leqslant t_{j} \leqslant 1\right\}
$$

and Supp $D=J_{+}^{\prime} \amalg J_{-}^{\prime}$. Let $a: L \rightarrow \bigoplus_{j \in J} k\left(Q_{j}\right)$ be a presentation over $k$ corresponding to a closed point of $\operatorname{StPres}(\partial \tilde{\xi}, J ; ?) \times^{T} \operatorname{orb}_{\xi}(D)$. Then it is easy to see that its restriction gives rise to a strict presentation

$$
a^{\prime}: L\left(-\sum_{j \in J_{+}^{\prime}}\left[Q_{j}^{+}\right]-\sum_{j \in J_{-}^{\prime}}\left[Q_{j}^{-}\right]\right) \rightarrow \underset{j \in J-\text { Supp } D}{\bigoplus} k\left(Q_{j}\right)
$$

with $\operatorname{ker}\left(a^{\prime}\right)=\operatorname{ker}(a)$.

As we saw in the proof of Proposition 12.10, we see that $D$ belongs to $\bar{D}(\operatorname{ker} a)$. In particular, $\operatorname{ker}(a)$ is $\phi$-semistable.

(iv) By composing the morphism $\tilde{v}_{\xi}$ with $w_{\phi}: W_{\phi} \rightarrow \mathrm{Jac}_{\phi}(X)$ of Theorem 12.14, we get a morphism

$$
\tilde{u}_{\tilde{\xi}}=w_{\phi} \circ \tilde{v}_{\tilde{\xi}}: \operatorname{StPres}(\partial \tilde{\xi}, J ; ?) \times^{\tilde{T}} \operatorname{Temb}(\tilde{\xi}) \rightarrow \operatorname{Jac}_{\phi}(X)
$$


(v) The argument in (iii) actually shows that there exists an isomorphism

$$
\tilde{g_{\xi}}(D): \operatorname{StPres}(m(D), J-\operatorname{Supp} D ; ?) \stackrel{\sim}{\rightarrow} \operatorname{StPres}(\partial \tilde{\xi}, J ; ?) \times^{\tilde{T}} \text { ỡ } \mathrm{b}_{\xi}(D)
$$

with $m(D)=\partial b(D)-d(\operatorname{Supp} D) / 2$, which sends a strict presentation $a^{\prime}$ : $L^{\prime} \rightarrow \bigoplus_{j \in J-\operatorname{Supp} D} k\left(Q_{j}\right)$ to the presentation

$$
a: L^{\prime}\left(\sum_{j \in J_{+}^{\prime}}\left[Q_{j}^{+}\right]+\sum_{j \in J_{-}^{\prime}}\left[Q_{j}^{-}\right]\right) \rightarrow \bigoplus_{j \in J} k\left(Q_{j}\right) \text {. }
$$

(vi) Taking the quotient of these by $\operatorname{ker}[\tilde{T} \rightarrow T]$, we obtain the following: for $\xi=\rho(\tilde{\xi}) \in \mathrm{Sk}^{0}(\mathrm{Dec})$, a morphism

$$
u_{\xi}: \operatorname{Pic}_{X}^{\partial \xi} \times^{T} \operatorname{Temb}(\xi) \rightarrow \operatorname{Jac}_{\phi}(X)
$$

which depends only on the image $\bar{\xi}$ of $\xi$ in $\overline{\operatorname{Dec}}$, and for $N=\rho(D)$ in Dec with $\xi \in D \in K_{\phi}^{0}$, an isomorphism

$$
g_{\xi}(N): \operatorname{Pic}_{X(J-S u p p}^{m(\bar{N})} \stackrel{\sim}{\rightarrow} \operatorname{Pic}_{X}^{\partial \bar{\xi}} \times^{T} \operatorname{orb}_{\xi}(N) .
$$

The composition of $g_{\xi}(N)$ with the morphism $u_{\bar{\xi}}$ obviously coincides with the isomorphism

$$
\operatorname{Pic}_{X(J-\operatorname{Supp} \bar{N})}^{m(\bar{N})} \stackrel{\sim}{\rightarrow} \mathbf{O}(\bar{N}) \subset \mathrm{Jac}_{\phi}(X)
$$

of Proposition 12.17(ii). Here $\bar{N}$ is the image of $N$ in $\overline{\text { Dec. }}$

(vii) Let $U$ be the scheme obtained from $\amalg_{\bar{\xi} \in S K^{0}(\overline{D e c})} \operatorname{Pic}_{X}^{\partial \bar{\xi}} \times{ }^{T} \operatorname{Temb}(\bar{\xi})$ by the identification maps $g_{\xi}(N)$ with $N \ni \xi$ running over all $N \in$ Dec and their vertices. Then we have a morphism

$$
u: U \rightarrow \mathrm{Jac}_{\phi}(X)
$$

which is bijective by (vi), since $\operatorname{Jac}_{\phi}(X)$ is the disjoint union of $\mathbf{O}(\bar{N})$ with $\bar{N}$ running over $\overline{\mathrm{Dec}}$ by Proposition 12.17 .

(viii) We now claim that $u$ is an isomorphism. Certainly it is enough, by Theorem 11.4, to show that there is a $\operatorname{GL}(E)$-invariant morphism $R=$ $R_{\phi}(E / P) \rightarrow U$ whose composition with $u$ coincides with the quotient morphism $R \rightarrow \mathrm{Jac}_{\phi}(X)$. By Lemma 11.8, we see that the completion of local rings of $R$ are of the form

$$
k\left[\left[x_{1}, x_{1}^{\prime}, \ldots, x_{r}, x_{r}^{\prime}, y_{1}, \ldots, y_{s}\right]\right] /\left(x_{1} . x_{1}^{\prime}, \ldots, x_{r} . x_{r}^{\prime}\right) .
$$

In particular, the normalization of $R$ is smooth. Let $S$ be a component of the normalization of $R$ and let $F$ be the restriction to $X \times S$ of $G \otimes P_{1}^{*} \theta_{X}(-\tilde{n})$. Since $S$ is irreducible and smooth, it is not hard to see that there exists a presentation $a: L \rightarrow N$ over $S$ such that $\operatorname{ker}(a)=F$ and that $\operatorname{deg} L=\partial \tilde{\xi}$ for a $\tilde{\xi}$ in $\operatorname{Sk}^{0}\left(K_{\phi}^{0}\right)$. Thus we have a morphism $S \rightarrow \operatorname{StPres}(\partial \tilde{\xi}, J ; ?) \times{ }^{\tilde{T}} \tilde{\operatorname{Temb}}(\tilde{\xi})$. Composing this with $u_{\xi}$, we get a morphism $S \rightarrow U$. It is obviously independent of the choice of the presentation $a$. Hence we have a morphism from the 
normalization of $R$ to $U$, which induces a GL(E)-invariant morphism from $R$ to $U$.

EXAMPLES. Let us consider the cases where $X$ is a stable curve of genus 2 in the sense of Deligne-Mumford [4].

(0) $X$ is a nonsingular irreducible. In this case, $\operatorname{Jac}_{\phi}(X)=\operatorname{Pic}_{X}^{0}$.

(1) $X$ is irreducible with one double point $Q$ and with $\tilde{X}$ an elliptic curve. As we saw in Example 13.1(1), we see that $\phi=0, H^{\prime}=H$ and $\operatorname{Pic}_{X}^{0} \times^{T}$ Temb(0) is a $\mathbf{P}_{1}$-bundle over $\mathbf{P i c}_{\tilde{X}}^{0}$, which can be identified, noncanonically, with

$$
\mathbf{P}\left(\theta_{\tilde{x}}\left(\left[Q^{+}\right]\right) \oplus \theta_{\tilde{x}}\left(\left[Q^{-}\right]\right)\right) \text {. }
$$

Let $N=\left\{t e_{1} ; 0 \leqslant t \leqslant 1\right\}$. Then $N$ is a 1 -dimensional Namikawa polyhedron, uniquely determined up to $H^{\prime}$-translation. $\mathbf{O}(\tilde{N})$ is canonically isomorphic to $\operatorname{Pic}_{\tilde{X}}^{-1} \cong \tilde{X}$. Two points in $\operatorname{StPres}(0, J ; ?) \times^{T} \operatorname{Temb}(0)$ corresponding to presentations

$$
a: L \rightarrow L\left(Q^{+}\right) \cong k(Q), \quad a^{\prime}: L^{\prime} \rightarrow L^{\prime}\left(Q^{-}\right) \cong k(Q)
$$

go into the same point of $\mathbf{O}(\bar{N})$ if and only if $L\left(-\left[Q^{+}\right]\right)$and $L^{\prime}\left(-\left[Q^{-}\right]\right)$are $\Theta_{\tilde{X}}$-isomorphic. We conclude that $\operatorname{Jac}_{\phi}(X)$ is obtained by identifying the 0 -section and the $\infty$-section of the $\mathbf{P}_{1}$-bundle $\mathbf{P}\left(\theta_{\tilde{X}}\left[Q^{+}\right]\right) \oplus \theta_{\tilde{X}}\left(\left[Q^{-}\right]\right)$over $\tilde{X}$ via the translation in $\tilde{X}$ by its point $Q^{+}-Q^{-}$(see Figure 33).

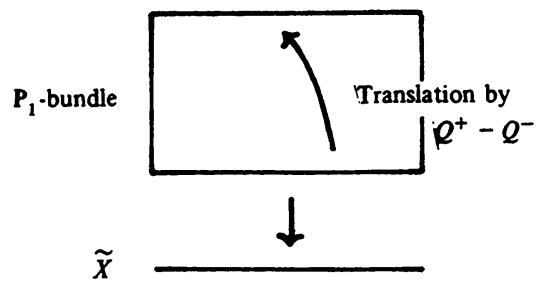

FIGURE 33

(2) $X$ is irreducible with two double points $\left\{Q_{1}, Q_{2}\right\}$ and with $\tilde{X}=\mathbf{P}_{1}$. As we saw in Example 13.1(2), we see that $\phi=0, H^{\prime}=H$ and $\operatorname{StPres}(0, J$; ?) $\times^{T} \operatorname{Temb}(0)=\mathbf{P}_{1} \times \mathbf{P}_{1}$. Let $N=\left\{t_{1} e_{1}+t_{2} e_{2} ; 0 \leqslant t_{j} \leqslant 1\right\}, N_{1}=\left\{t_{1} e_{1}\right.$; $\left.0 \leqslant t_{1} \leqslant 1\right\}$ and $N_{2}=\left\{t_{2} e_{2} ; 0 \leqslant t_{2} \leqslant 1\right\}$. Then $\overline{\text { Dec }}$ consists of $\bar{N}, \bar{N}_{1}, \bar{N}_{2}$ and $\overline{0}$. The point $\mathbf{O}(\bar{N})$ in $\operatorname{Jac}_{\phi}(X)$ corresponds to the $\theta_{X}$-module $\theta_{\mathbf{P}_{1}}(-2)=$ $\theta_{\bar{X}}\left(-\left[Q_{1}^{\alpha}\right]-\left[Q_{2}^{\beta}\right]\right)$ with $\alpha, \beta= \pm$. Consider presentations $a, A: \theta_{\bar{x}} \rightarrow k\left(Q_{1}\right)$ $\oplus k\left(Q_{2}\right)$ where $a$ belongs to orb $0\left(N_{2}\right)=\mathbf{P}_{1} \times 0$ and $A$ belongs to orb $0\left(-N_{2}\right)$ $=\mathbf{P}_{1} \times \infty$. Thus there exist elements $a^{+}, a^{-}, A^{+}$and $A^{-}$in $k^{*}$ such that $a(f)=a^{+} f\left(Q_{1}^{+}\right)+a^{-} f\left(Q_{1}^{-}\right)$and $A(f)=A^{+} f\left(Q_{1}^{+}\right)+A^{-} f\left(Q_{1}^{-}\right)$for $f$ in $\Theta_{\bar{x}, Q_{1}}$, and $a(f)=f\left(Q_{2}^{-}\right)$and $A(f)=f\left(Q_{2}^{+}\right)$for $f$ in $\Theta_{\bar{x}, Q_{2}}$. Here $a^{+} / a^{-}$and $A^{+} / A^{-}$are the coordinates of $a$ and $A$, respectively, in the first factor $\mathbf{P}_{1}$. 
Thus the corresponding strict presentations are

$$
a^{\prime}: \theta_{\bar{X}}\left(-\left[Q_{2}^{-}\right]\right) \rightarrow k\left(Q_{1}\right), \quad A^{\prime}: \theta_{\bar{X}}\left(-\left[Q_{2}^{+}\right]\right) \rightarrow k\left(Q_{1}\right)
$$

with $a^{\prime}(f)=a(f)$ and $A^{\prime}(f)=A(f)$ for $f$ in $\theta_{\tilde{X}, Q_{1}}$. Obviously, $\operatorname{ker}\left(a^{\prime}\right)$ and $\operatorname{ker}\left(A^{\prime}\right)$ are $\theta_{X}$-isomorphic if and only if there exists an $\theta_{\bar{X}}$-isomorphism $b$ : $\vartheta_{\tilde{X}}\left(-\left[Q_{2}^{+}\right]\right) \stackrel{\mathcal{X}}{\rightarrow} \vartheta_{\tilde{X}}\left(-\left[Q_{2}^{-}\right]\right)$and $c$ in $k^{*}$ such that $a^{\prime} \circ b=c A^{\prime}$. Since $\tilde{X}=\mathbf{P}_{1}$, there exists a function $z$ on $\tilde{X}$ with $(z)=\left[Q_{2}^{+}\right]-\left[Q_{2}^{-}\right]$. The $\theta_{\tilde{X}}$-isomorphism $b$ differs from the multiplication by $z$ only by a scalar multiplication. Thus $\operatorname{ker}\left(a^{\prime}\right)$ and $\operatorname{ker}\left(A^{\prime}\right)$ are $\theta_{X}$-isomorphic if and only if $\left(a^{+} / a^{-}\right)\left(z\left(Q_{1}^{+}\right) / z\left(Q_{1}^{-}\right)\right)$ $=A^{+} / A^{-}$. Note that $r=z\left(Q_{1}^{+}\right) / z\left(Q_{1}^{-}\right)$is the cross ratio of $\left\{Q_{2}^{-}, Q_{2}^{+}, Q_{1}^{-}, Q_{1}^{+}\right\}$. We conclude that $\mathrm{Jac}_{\phi}(X)$ is obtained from $\mathbf{P}_{1} \times \mathbf{P}_{1}$ by identifying $(x, 0)$ with $(r x, \infty)$ and $(0, y)$ with $(\infty, r y)$ (see Figure 34 ).

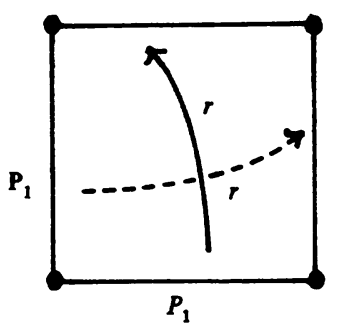

FIGURE 34

(3) Finally $X$ has two nonsingular rational components meeting at three points. As in Example 13.1(3), let us consider the following two cases:

(a) $\phi=0$. In this case $\operatorname{Jac}_{\phi}(X)$ is obtained from $\operatorname{Temb}\left(-\bar{e}_{1}\right)=\mathbf{P}_{2}$, $\operatorname{Temb}\left(\bar{e}_{1}\right)=\mathbf{P}_{2}$ and $\operatorname{Temb}(\overline{0})$ which is the blowing up of $\mathbf{P}_{2}$ along the three coordinate vertices, by the identifications, as in Figure 35 , where $\alpha, \beta, \gamma, \delta, \varepsilon$ and $\zeta$ are all $\mathbf{P}_{1}$.

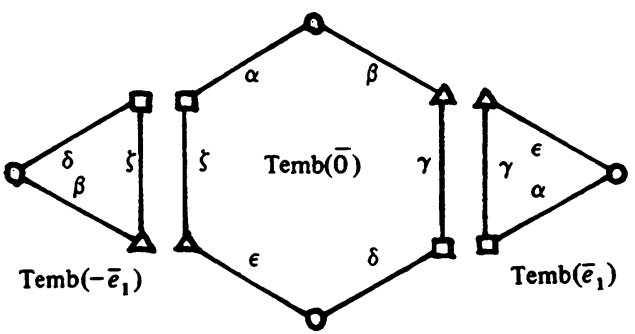

FIGURE 35

(b) $\phi=\left(v_{1}-v_{2}\right) / 2$. In this case, $\operatorname{Jac}_{\phi}(X)$ is obtained from $\operatorname{Temb}(\overline{0})=\mathbf{P}_{2}$ and $\operatorname{Temb}\left(\bar{e}_{1}\right)=\mathbf{P}_{2}$ by the identifications as in Figure 36, where $\alpha, \beta$ and $\gamma$ are $\mathbf{P}_{1}$. 


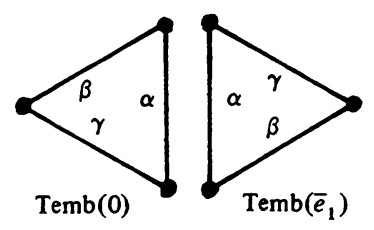

FIGURE 36

REMARK. The compactifications $\mathrm{Jac}_{\phi}(X)$ we obtained in (0). (1), (2) and (3a) appear in Mumford [22]. On the other hand, (0), (1), (2) and (3b) appear as stable quasi-abelian varieties in Nakamura [26] and Namikawa [27].

REMARK. Let us consider the following identifications in $\mathbf{P}_{1} \times \mathbf{P}_{1}$ :

$$
(x, 0) \sim(r x, \infty), \quad(0, y) \sim\left(\infty, r^{\prime} y\right)
$$

for fixed elements $r$ and $r^{\prime}$ in $k^{*}$ and $x, y$ running over points of $\mathbf{P}_{1}$. It was pointed out by Deligne to Namikawa that the variety obtained by these identifications is not projective if $r^{n} \neq r^{\prime n^{\prime}}$ for any pair $n, n^{\prime}$ of positive integers.

14. Relations with Raynaud's results. Let $S=\{\eta, s\}$ be the spectrum of a discrete valuation ring with the residue field $k(s)=k$. Consider a proper and flat family $Y$ of curves over $S$ such that $Y_{\eta}$ is a smooth connected complete curve over $k(\eta), Y_{s}=X=\cup_{i \in I} X_{i}$ is a reduced connected complete curve over $k$ with at most ordinary double points $\left\{Q_{j}\right\}_{j \in J}$.

In this case, the relative Picard functor $\mathrm{Pic}_{Y / S}$ need not be separated nor representable. Raynaud [29] takes its greatest separated quotient $Q=Q_{Y / S}$. $Q$ is then represented by a separated and smooth group scheme over $S$ (Proposition 8.0.1). Moreover, $Q^{\tau}$ is the Néron model of the Jacobian variety $\operatorname{Pic}_{Y_{\eta}}^{0}$ of the generic fiber $Y_{\eta}$, if $Y$ is factorial (Theorem 8.1.4).

For simplicity, let us assume that $S$ is Henselian and that $Y$ is a regular scheme.

Let $E$ be the subgroup of $\operatorname{Pic}(X)$ generated by

$$
\left\{\theta_{X} \otimes_{\theta_{Y}} \theta_{Y}\left(X_{i}\right) ; i \in I\right\} \text {. }
$$

Let $\operatorname{Pic}^{\text {tot.deg }}{ }^{0}(X)$ be the subgroup of $\operatorname{Pic}(X)$ consisting of $L \in \operatorname{Pic}(X)$ with total degree $\left[v(I), \operatorname{deg} \theta_{\tilde{X}} \otimes_{\theta_{x}} L\right]=0$. Then we get

$$
\begin{aligned}
Q_{s}(\operatorname{Spec} k) & =\operatorname{Pic}(X) / E, \\
\left(Q^{\tau}\right)_{s}(\operatorname{Spec} k) & =\operatorname{Pic}^{\text {tot.deg }} 0(X) / E .
\end{aligned}
$$

LEMMA 14.1. Let $i$ and $i^{\prime}$ be in $I$. Then the intersection number of $X_{i}$ and $X_{i^{\prime}}$ as divisors on $Y$, is given by

$$
\left(X_{i}, X_{i^{\prime}}\right)_{Y}=-\left(\delta v_{i}, \delta v_{i^{\prime}}\right)
$$


Proof. If $i \neq i^{\prime}$, then obviously $\left(X_{i}, X_{i^{\prime}}\right)_{Y}$ equals the number of edges $j \in J$ joining $i$ and $i^{\prime}$, regardless of the orientation, which is easily seen to coincide with $-\sum_{j \in J}\left[v_{i}, \partial e_{j}\right]\left[v_{i^{\prime}}, \partial e_{j}\right]=-\left(\delta v_{i}, \delta v_{i^{\prime}}\right)$. Note that $\left(X_{i}, \sum_{i^{\prime} \in I} X_{i^{\prime}}\right)_{Y}=0$ since the second member is the fiber of $Y$ over $s$. Thus

$$
\left(X_{i}, X_{i}\right)_{Y}=-\sum_{i^{\prime} \neq i}\left(X_{i}, X_{i^{\prime}}\right)_{Y}=\left(\delta v_{i}, \delta v(I-\{i\})\right)=-\left(\delta v_{i}, \delta v_{i}\right)
$$

since $\delta v(I)=0$.

CoROllaRY 14.2. For $i$ in $I$, the degree of the pull-back of $\theta_{X} \otimes_{\theta_{Y}} \theta_{Y}\left(X_{i}\right)$ to $\tilde{X}$ is given by

$$
\operatorname{deg}\left(\theta_{\bar{X}} \otimes_{\theta_{Y}} \theta_{Y}\left(X_{i}\right)\right)=-\partial \delta v_{i}
$$

Proof. By the definition in $\S 10$, the degree is equal to $\sum_{i^{\prime} \in I}\left(X_{i}, X_{i^{\prime}}\right)_{Y} v_{i}$, which is equal, by Lemma 14.1, to

$$
-\sum_{i^{\prime} \in I}\left(\delta v_{i}, \delta v_{i^{\prime}}\right) v_{i^{\prime}}=-\sum_{i^{\prime} \in I}\left[\partial \delta v_{i}, v_{i^{\prime}}\right] v_{i^{\prime}}=-\partial \delta v_{i}
$$

REMARK. We see that $-\partial \delta: C_{0}(\Gamma(X), \mathbf{Z}) \rightarrow C_{0}(\Gamma(X), \mathbf{Z})$ is represented by the intersection matrix of $X_{i}$ 's on $Y$.

Obviously, $E \cap \operatorname{Pic}^{0}(X)=\{0\}$. On the other hand, deg induces an isomorphism from $\mathrm{Pic}^{\text {tot.deg }}{ }^{0}(X) / \mathrm{Pic}^{0}(X)$ to $\partial C_{1}(\Gamma(X), \mathbf{Z})$. Thus in view of Kirchhoff-Trent's theorem in $\$ 4$, we conclude:

Proposition 14.3. There is a canonical isomorphism

$$
\left(Q_{Y / S}^{\tau}\right)_{s}(\operatorname{Spec} k) / \operatorname{Pic}^{0}(X) \cong \partial C_{1}(\Gamma(X), \mathbf{Z}) / \partial \delta C_{0}(\Gamma(X), \mathbf{Z})
$$

The order of these groups are equal to complexity $(\Gamma(X))$ which is the number of spanning trees in $\Gamma(X)$. In particular, the group scheme $\left(Q_{Y / s}^{\tau}\right)_{s}$ over $k$ is independent of the particular family $Y / S$ containing $X$ as the special fiber.

COROLLARY 14.4. For a nondegenerate $\phi, \mathrm{Jac}_{\phi}(X)$ is a compactification of the special fiber $\left(Q_{Y / S}^{\tau}\right)_{s}$ of the Néron model $Q_{Y / S}^{\tau}$ of $\operatorname{Pic}_{Y_{\eta}}^{0}$.

REMARK. As was shown by Ishida [36], the geometric invariant theory enables us to construct projective schemes $\operatorname{Jac}_{\phi}(Y / S)$ over $S$, which contain the Néron model $Q_{Y / S}^{\tau}$ itself and whose special fiber is the compactification given in Corollary 14.4.

Appendix. A lemma in M. Artin [1]. We state and prove in this Appendix a modification of a lemma in Artin [1, Proposition 31] used in connection with the deformation of singularities. This version can be found also in D'Souza [7]. See also [EGA, Chapitre $O_{1 V}$, Proposition 19.1.10]. 
LEMMA. Let $A \rightarrow B$ be a local homomorphism of noetherian local rings. Let $N$ and $L$ be $B$-modules of finite type with $L A$-flat. Then a B-homomorphism $f$ : $N \rightarrow L$ is injective with $A$-flat cokernel if and only if

$$
f \otimes_{A} k: N \otimes_{A} k \rightarrow L \otimes_{A} k
$$

is injective, where $k$ is the residue field of $A$.

Proof. The "only if" part is obvious. We now prove the "if" part. Let $K=\operatorname{Image}(f)$ and $C=\operatorname{Coker}(f)$. We thus have exact sequences $0 \rightarrow K \rightarrow L$ $\rightarrow C \rightarrow 0$ and $N \rightarrow K \rightarrow 0$. By tensoring $k$, we get

$$
\begin{gathered}
0 \rightarrow \operatorname{Tor}_{1}^{A}(C, k) \rightarrow K \otimes_{A} k \rightarrow L \otimes_{A} k \rightarrow C \otimes_{A} k \rightarrow 0 \\
\qquad \bigotimes_{A} k=N \otimes_{A} k
\end{gathered}
$$

Obviously, we conclude that $N \otimes_{A} k \stackrel{\sim}{\rightarrow} K \otimes_{A} k$ is an isomorphism and $K \otimes_{A} k \rightarrow L \otimes_{A} k$ is injective. Hence first of all we see that $\operatorname{Tor}_{1}^{A}(C, k)=0$, i.e. $C$ is $A$-flat by [SGA, 60/61, Exposé IV, Theorem 5.6]. Since $L$ and $C$ are $A$-flat, we conclude that $K$ is $A$-flat. Let $N^{\prime}$ be the kernel of the surjective map $N \rightarrow K$. Since $N \otimes_{A} k \stackrel{\sim}{\rightarrow} K \otimes_{A} k$ is an isomorphism, and since $K$ is $A$-flat, we see that $N^{\prime} \otimes_{A} k=0$. Hence by Krull-Azumaya's (i.e. the so-called Nakayama) lemma, we get $N^{\prime}=0$.

REMARK 1. We use $B$ in the above lemma only for the finiteness assumption on $L$ and $N$, as usual, so that we can use Krull-Azumaya's lemma.

REMARK 2. The original form of this lemma is the following: Let

$$
(L): L_{2} \rightarrow L_{1} \rightarrow L_{0} \rightarrow F \rightarrow 0
$$

be a complex of $B$-modules of finite type, which is exact at $L_{0}$ and $F$ with $L_{0}, L_{1}$ and $L_{2} B$-free. If $(L) \otimes_{A} k$ is exact, then $(L)$ is exact and $F$ is $A$-flat. This version follows from ours by taking $N=\operatorname{Coker}\left[L_{2} \rightarrow L_{1}\right]$ and $L=L_{0}$.

COROLlaRY. Let $A \rightarrow B$ be a flat local homomorphism of noetherian local rings. Let $N$ be a $B$-module of finite type which is $A$-flat and satisfies $\operatorname{Ext}_{\bar{B}}^{1}(\bar{N}, \bar{B})=0$, where $\bar{B}=B \otimes_{A} k$ and $\bar{N}=N \otimes_{A} k$ for the residue field $k$ of $A$. Then $\operatorname{Hom}_{B}(N, B)$ is $A$-flat and $\operatorname{Hom}_{B}(N, B) \otimes_{A} k=\operatorname{Hom}_{\bar{B}}(\bar{N}, \bar{B})$.

Proof. Let

$$
(L): L_{2} \rightarrow L_{1} \rightarrow L_{0} \rightarrow N \rightarrow 0
$$

be a resolution of $N$ by free $B$-modules of finite type. Since $N$ is $A$-flat, we see 
that

$$
(L) \otimes_{A} k: \bar{L}_{2} \rightarrow \bar{L}_{1} \rightarrow \bar{L}_{0} \rightarrow \bar{N} \rightarrow 0
$$

is exact. Thus it is a resolution of $\bar{N}$ by free $\bar{B}$-modules of finite type. Let $L_{i}^{*}=\operatorname{Hom}_{B}\left(L_{i}, B\right)$. Then $\bar{L}_{i}^{*}=L_{i}^{*} \otimes_{A} k=\operatorname{Hom}_{\bar{B}}\left(\bar{L}_{i}, \bar{B}\right)$. Since $\operatorname{Ext}_{\bar{B}}^{1}(\bar{N}, \bar{B})$ $=0$ by assumption, we see that

$$
\left\{(L) \otimes_{A} k\right\}^{*}: 0 \rightarrow \operatorname{Hom}_{\bar{B}}(\bar{N}, \bar{B}) \rightarrow \bar{L}_{0}^{*} \rightarrow \bar{L}_{1}^{*} \rightarrow \bar{L}_{2}^{*}
$$

is exact. Consider now the complex

$$
\left(L^{*}\right): 0 \rightarrow \operatorname{Hom}_{B}(N, B) \rightarrow L_{0}^{*} \rightarrow L_{1}^{*} \rightarrow L_{2}^{*} .
$$

Obviously $\left(L^{*}\right)$ is exact at $\operatorname{Hom}_{B}(N, B)$ and $L_{0}^{*}$. Consider

$$
f: \operatorname{Coker}\left[L_{0}^{*} \rightarrow L_{1}^{*}\right] \rightarrow L_{2}^{*} \text {. }
$$

Then $f \otimes_{A} k$ is injective, since $\left\{(L) \otimes_{A} k\right\}^{*}$ is exact. Hence by the Lemma we conclude that $f$ is injective with $A$-flat cokernel. Since $L_{2}^{*}$ is $A$-flat, we see that Coker $\left[L_{0}^{*} \rightarrow L_{1}^{*}\right]$ is also $A$-flat. Since $L_{0}^{*}$ and $L_{1}^{*}$ are $A$-flat, we conclude that $\operatorname{Hom}_{B}(N, B)$ is $A$-flat.

REMARK 3. We apply this corollary when $B$ is $A$-flat and $\bar{B}=B \otimes_{A} k$ is a local ring of a reduced curve with at most ordinary double points. Moreover, $\bar{N}=N \otimes_{A} k$ is $\bar{B}$-torsion-free and of rank one on each component, i.e. $\bar{N}$ is either $\bar{B}$ or the maximal ideal of $\bar{B}$. In this case, we see immediately that $\operatorname{Ext}_{\bar{B}}^{1}(\bar{N}, \bar{B})=0$.

\section{REFERENCES}

1. M. Artin, Lectures on deformations of singularities, Tata Inst. of Fundamental Research, No 54, Bombay, 1976.

2. C. Berge, Graphs and hypergraphs, North-Holland, Amsterdam, 1970.

3. P. R. Bryant, Graph theory applied to electrical networks, Graph Theory and Theoretical Physics (F. Harary, ed.), Academic Press, New York, 1967, pp. 111-137.

4. P. Deligne and D. Mumford, The irreducibility of the space of curves of given genus, Publ. Math. Inst. Hautes Etudes Sci. 36 (1969), 75-10.

5. P. Deligne and M. Rapoport, Les schemas de mochules de courbes elliptique, Modular Functions of One Variable. II, Lecture Notes in Math., vol. 349, Springer-Verlag, New York, 1973.

EGA. J. Dieudonné and A. Grothendieck, Éléments de géométrie algébrique, Publ. Math. Inst. Hautes Études Sci., Nos. 4, 8, 11, 17, 20, 24, 28, 32 (1960-1967).

6. T. J. Dickson, On Voronoi reduction of positioe definite quadratic forms, J. Number Theory 4 (1972), 330-341.

7. C. D'Souza, Compactification of generalized Jacobian, Thesis, TIFR and Bombay Univ. 1974; Astérisque (to appear).

8. D. Gieseker, On the moduli of vector bundles on an algebraic surface, Ann. of Math. 106 (1977), 45-60. 
9.

FGA. A. Grothendieck, Fondements de la géométrie algébrique, Extraits du Séminaire Bourbaki 1957-1962, Paris, 1962.

10. W. Haboush, Reductive groups are geometrically reductive, Ann. of Math. (2) 102 (1975), 67-83.

11. F. Harary, Graph theory, Addison-Wesley, Reading, Mass., 1969.

12. A. J. Hoffman and J. B. Kruskal, Integral boundary points of convex polyhedra, Linear Inequalities and Related Systems (Kuhn and Tucker, ed.), Ann. of Math. Studies, vol. 38, Princeton Univ. Press, Princeton, N. J., 1956, pp. 223-246.

13. I. Heller and C. B. Tompkins, An extension of a theorem of Dantzig's, Linear Inequalities and Related Systems, Ann. of Math. Studies, vol. 38, Princeton Univ. Press, Princeton, N. J., 1956, pp. 247-254.

14. J. Igusa, Fiber systems of Jacobian varieties. I, II, III, Amer. J. Math. 78 (1956), 177-199; 745-760; 81 (1959), 453-475.

15. S. C. Langton, Valuative criteria for families of vector bundles on algebraic varieties, Ann. of Math. (2) 101 (1975), 88-110.

16. M. Maruyama, Stable vector bundles on an algebraic surface, Nagoya Math. J. 58 (1975), 25-68.

17. K. Miyake and T. Oda, Almost homogeneous algebraic varieties under algebraic torus action, Manifolds, Tokyo 1973, Univ. of Tokyo Press, Tokyo, 1974, pp. 373-381. See also: T. Oda, Lectures on torus embeddings and applications (Based on joint work with Katsuya Miyake), Tata Inst. of Fundamental Research, No. 58, Bombay, 1978.

18. A. L. Mayer, Compactification of the variety of moduli of curves, Lectures $2 \& 3$, Seminar on degeneration of algebraic varieties (mimeographed notes), Inst. for Advanced Study, Princeton, N. J., 1969/70.

19. D. Mumford, Further comments on boundary points (mimeographed notes), AMS Summer School at Woods Hole, 1964.

20. __, Geometric invariant theory, Springer-Verlag, New York, 1965.

21. ___ Abelian varieties, Oxford Univ. Press, Bombay, 1970.

22. __ An analytic construction of degenerating abelian varieties over complete rings, Compositio Math. 24 (1972), 239-272.

23. Curves and their Jacobians, Univ. of Michigan Press, Ann Arbor, 1975.

24. D. Mumford et al., Toroidal embeddings. I, Lecture Notes in Math., vol. 339, SpringerVerlag, New York, 1973.

25. D. Mumford and P. Newstead, Periods of a moduli space of bundies on curves, Amer. J. Math. 90 (1968), 1200-1208.

26. I. Nakamura, On moduli of stable quasi-abelian varieties, Nagoya Math. J. 58 (1975), 149-214.

27. Y. Namikawa, A new compactification of the Siegel space and degeneration of abelian varieties. I, II, Math. Ann. 221 (1976), 97-141, 201-241.

28. M. S. Narashiman and C. S. Seshadri, Stable and unitary vector bundles on a compact Riemann surface, Ann. of Math. (2) 82 (1965), 540-567.

29. M. Raynaud, Spécialization du foncteur de Picard, Publ. Math. Inst. Hautes Etudes Sci. 38 (1970), 27-76.

30. C. A. Rogers, Packings and coverings, Cambridge Tracts in Math. and Math. Phys., no 54, Cambridge Univ. Press, New York, 1964.

31. C. S. Seshadri, Space of unitary vector bundles on a compact Riemann surface, Ann. of Math. (2) 85 (1967), 303-336.

32. , Mumford's conjecture for GL(2) and applications, (Internat. Colloq. TIFR, Bombay, 1968), Algebraic Geometry, Oxford Univ. Press, London, 1969, pp. 347-371.

33. __ Theory of moduli, Algebraic Geometry-Arcata 1974, Proc. Sympos. Pure Math., vol. 29, Amer. Math. Soc., Providence, R. I., 1975, pp. 263-304. 
34. G. Voronoi, Nouvelles applications des paramètres continues et théorie des formes quadratiques. I, II-1, II-2, J. Reine Angew. Math. 133 (1908), 97-178; 134 (1908), 198-287; 136 (1909), 67-181.

35. A. Ramanathan, Stable principal bundles on a compact Riemann surface-Construction of moduli space, Thesis, TIFR and Bombay Univ., 1976.

SGA. A. Grothendieck, Séminaire de géométrie algébrique, Inst. Hautes Études Sci., 1960/61.

36. M. Ishida, Compactifications of a family of generalized Jacobian varieties, (Proc. Internat. Sympos. Algebraic Geometry, Kyoto, January, 1977, M. Nagata, ed.), Kinokuniya, Tokyo, 1978, pp. 503-524.

MAThematical INSTtTUTE, TÔHOKU UNIVERSTTY, SENDA, 980 JAPAN

TATa Institute of Fundamental Research, Hom BhabHa ROAD, BOMBay 400005 Indu 